\title{
Small Commercial Building Re-Tuning: A Primer
}

KA Cort

DJ Hostick

RM Underhill

September 2013
N Fernandez

$S$ Katipamula

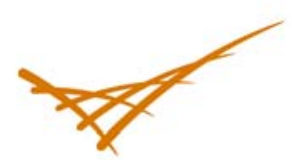

Pacific Northwest NATIONAL LABORATORY

Proudly Operated by Battelle Since 1965 


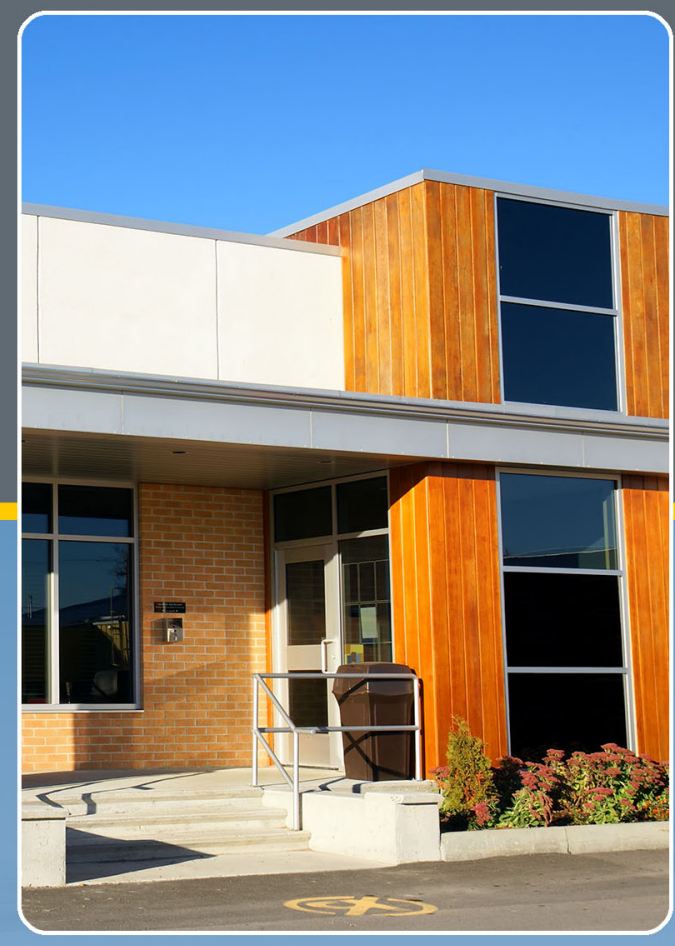

\section{Small Commercial Building Re-Tuning: A Primer}




\title{
DISCLAIMER
}

This report was prepared as an account of work sponsored by an agency of the United States Government. Neither the United States Government nor any agency thereof, nor Battelle Memorial Institute, nor any of their employees, makes any warranty, express or implied, or assumes any legal liability or responsibility for the accuracy, completeness, or usefulness of any information, apparatus, product, or process disclosed, or represents that its use would not infringe privately owned rights. Reference herein to any specific commercial product, process, or service by trade name, trademark, manufacturer, or otherwise does not necessarily constitute or imply its endorsement, recommendation, or favoring by the United States Government or any agency thereof, or Battelle Memorial Institute. The views and opinions of authors expressed herein do not necessarily state or reflect those of the United States Government or any agency thereof.

\author{
PACIFIC NORTHWEST NATIONAL LABORATORY \\ operated by \\ BATTELLE \\ for the \\ UNITED STATES DEPARTMENT OF ENERGY \\ under Contract DE-AC05-76RL01830
}

Printed in the United States of America
Available to DOE and DOE contractors from the Office of Scientific and Technical Information,
P.O. Box 62, Oak Ridge, TN 37831-0062;
ph: (865) 576-8401
fax: (865) 576-5728
email: reports@adonis.osti.gov

\author{
Available to the public from the National Technical Information Service, \\ U.S. Department of Commerce, 5285 Port Royal Rd., Springfield, VA 22161 \\ ph: (800) 553-6847 \\ fax: $(703) 605-6900$ \\ email: orders@ntis.fedworld.gov \\ online ordering: http://www.ntis.gov/ordering.htm
}

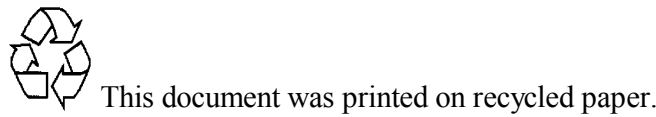

$(9 / 2003)$ 


\section{Small Commercial Building Re-tuning: A Primer}

KA Cort

N Fernandez

DJ Hostick

S Katipamula (Principal Investigator)

RM Underhill

September 2013

Prepared for

U.S. Department of Energy

under Contract DE-AC05-76RL01830

Pacific Northwest National Laboratory

Richland, Washington 99352 
Small Commercial Building Re-tuning: A Primer 


\section{CONTENTS}

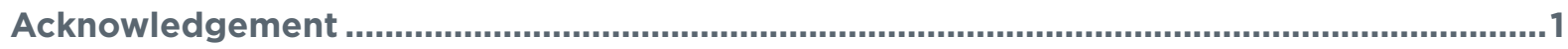

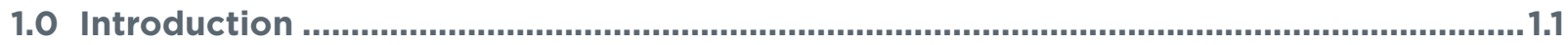

2.0 How to Use the Primer ............................................................................................... 2.1

3.0 Introduction to Building Energy................................................................................................ 3.1

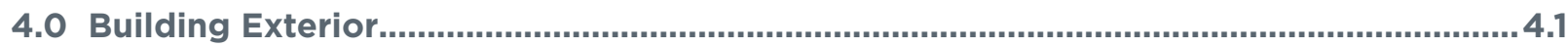

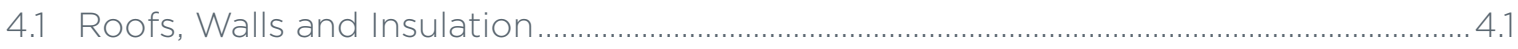

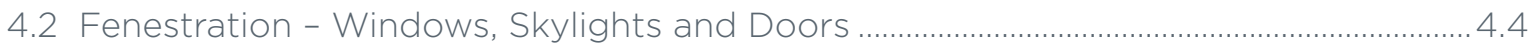

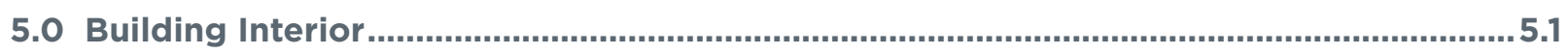

6.0 Heating, Ventilation, and Air-Conditioning ................................................................. 6.1

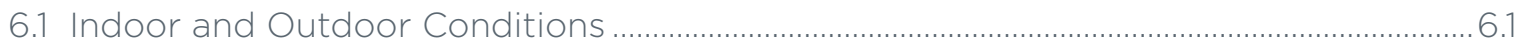

6.2 Sources of Indoor Heat, Moisture and Contaminants............................................................ 6.2

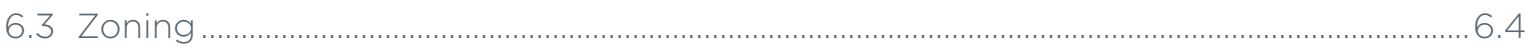

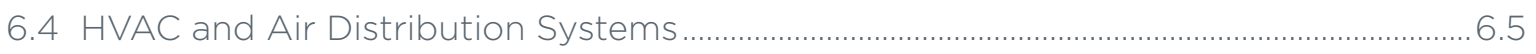

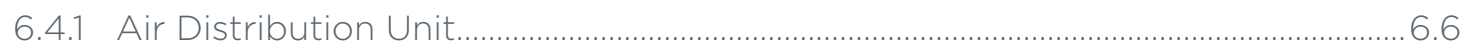

6.4.2 Outdoor Air Intake/Exhaust ........................................................................................ 6.6

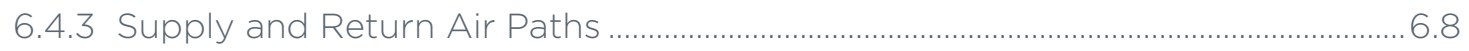

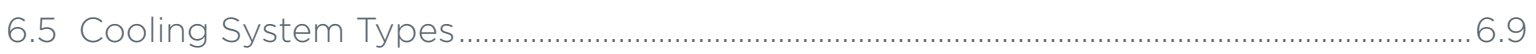

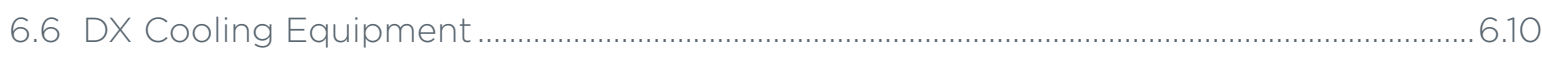

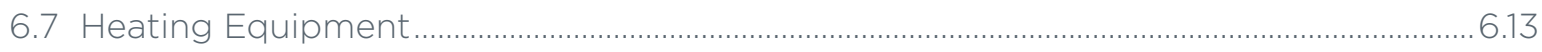

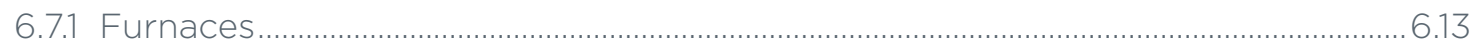

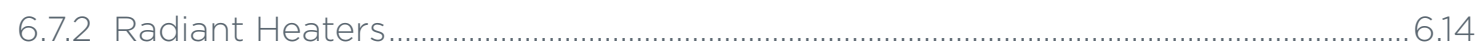

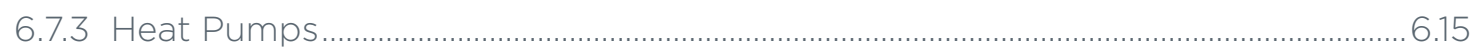

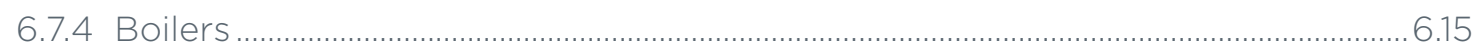

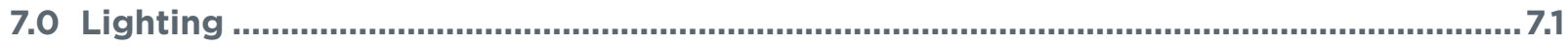

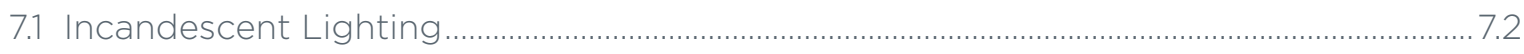

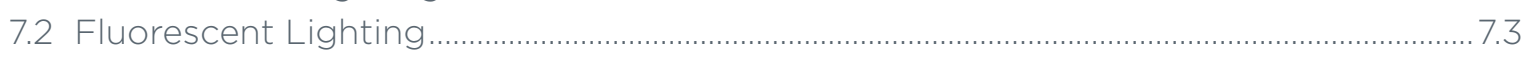

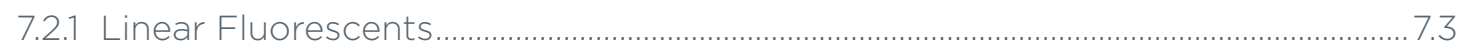

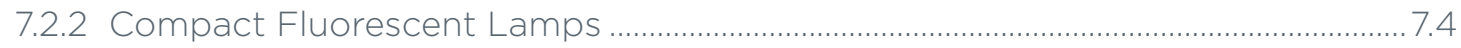

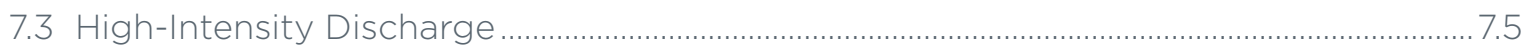

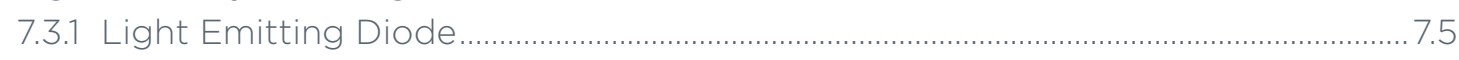

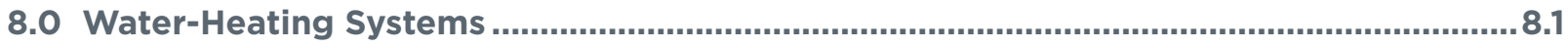

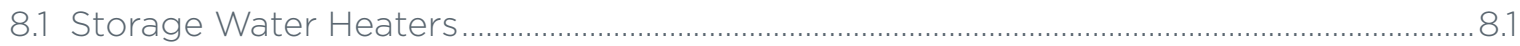

8.2 Heat Pump Water Heaters and Desuperheaters ...................................................................... 8.2

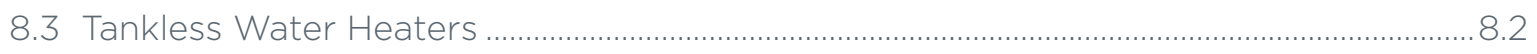

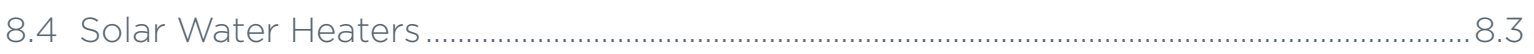

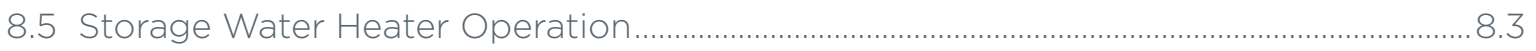




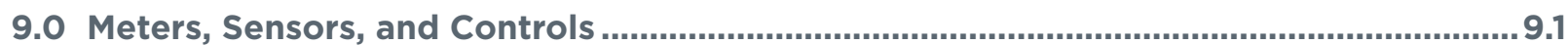

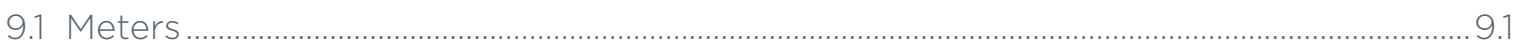

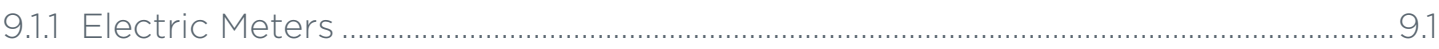

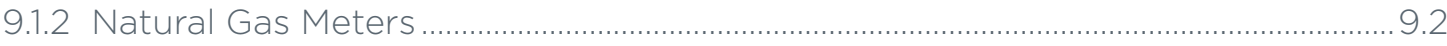

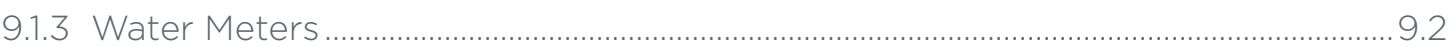

9.2 Sensors and Control Systems …............................................................................................. 9

9.2.1 Temperature Sensors and Temperature Control Systems ......................................9.3

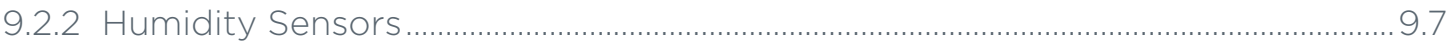

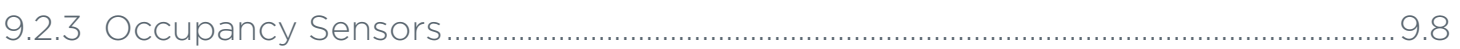

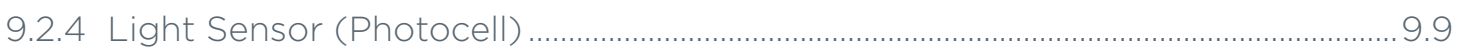

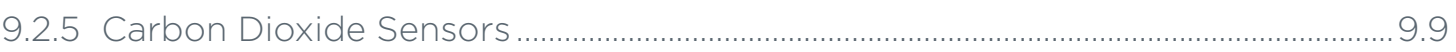

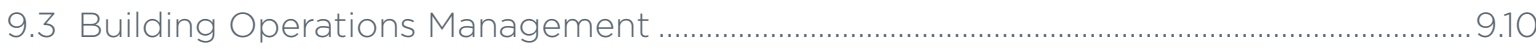

9.3.1 Proactive Versus Reactive Management ................................................................. 9.10

9.3.2 Morning Warm-Up and Cool-Down ............................................................................ 9.12

10.0 Basic Tools Needed for Re-tuning.....................................................................10.1

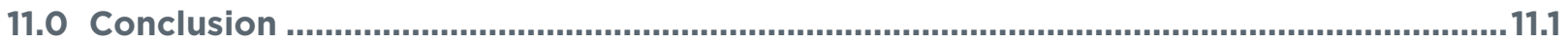

Appendix A: Glossary of Terms .....................................................................................

Appendix B: More Detail of HVAC System Terms, Definitions,

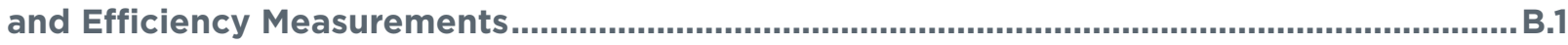




\section{FIGURES}

Figure 3.1. Commercial building components. ...................................................................................... 3.1

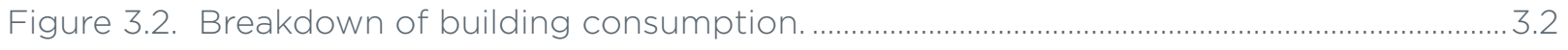

Figure 4.1. Examples of different styles of commercial roofs............................................................. 4.2

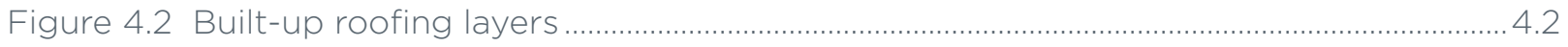

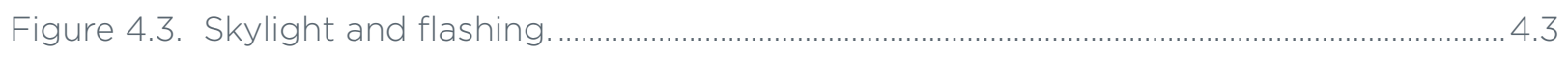

Figure 4.4. Installation of roof curb around a chimney.................................................................... 4.3

Figure 4.5. Heat trace diagram and typical control box ............................................................... 4.3

Figure 4.6. Examples of caulk around windows and doors with air gaps ...................................4.4

Figure 4.7. Windows can be single-, double- or triple-paned glass................................................ 4.5

Figure 5.1. Hourly load profile in grocery store (weekday in summer).............................................5.1

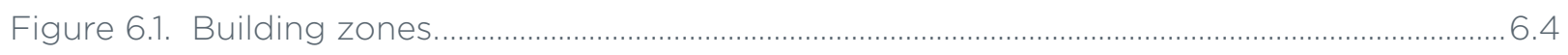

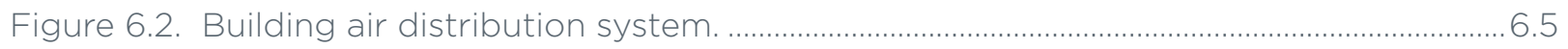

Figure 6.3. Outdoor air intake, exhaust and mixing box for a system with a return fan. ..........6.7

Figure 6.4. Outdoor air intake and mixing box for a system with no return fan.........................6.8

Figure 6.5. Examples of duct arrangements in commercial buildings. ............................................ 6.8

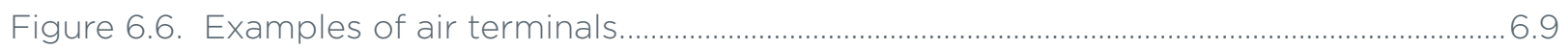

Figure 6.7. Example of several packaged rooftop units...................................................................... 6.10

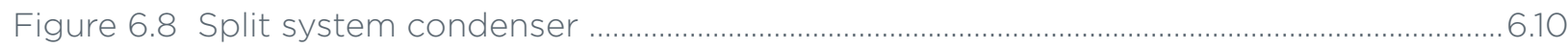

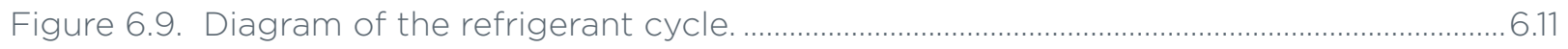

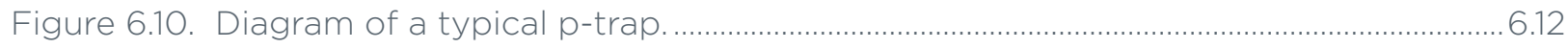

Figure 6.11. Heating chamber inside a gas furnace. ............................................................................... 6.14

Figure 6.12. Radiant baseboard heater and convective heater ....................................................... 6.14

Figure 6.13. Diagram of the heat pump vapor compression refrigeration cycle............................6.15

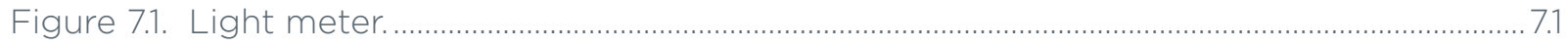

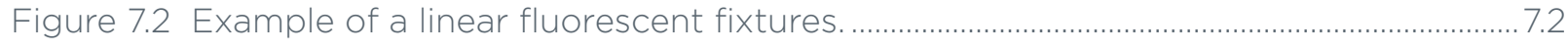

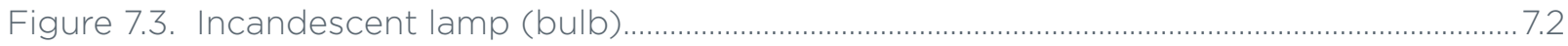

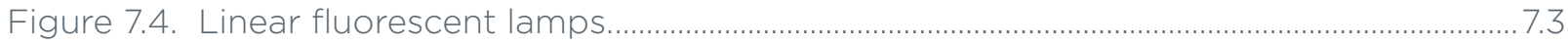

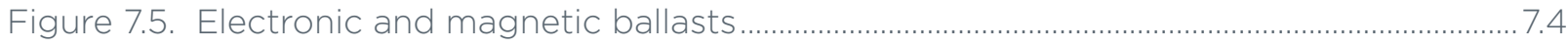

Figure 7.6. Examples of compact fluorescent lamps. ……………………………………................. 7.4

Figure 7.7. Example of HID lighting (high pressure sodium) in a parking lot. ..............................7.5

Figure 7.8. Examples and applications of LED lighting in commercial buildings.........................7.6

Figure 8.1. Common storage water heater tank ....................................................................................... 8.1

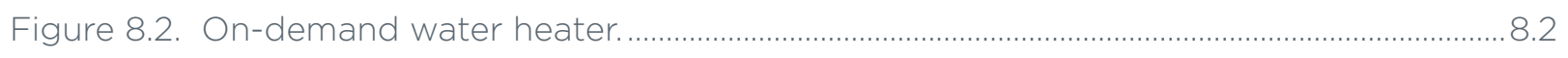


Small Commercial Building Re-tuning: A Primer

Figure 8.3. Solar panels and storage tank associated with a solar water-heating system........8.3

Figure 8.4. Diagram of a gas water heater.................................................................................................. 8.4

Figure 8.5. Water temperature controls and thermometer on an intake pipe.............................8.4

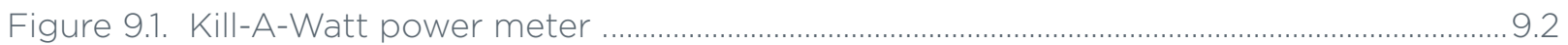

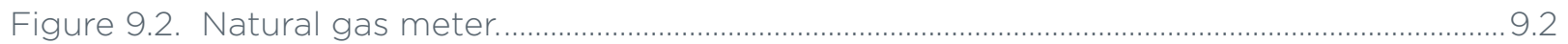

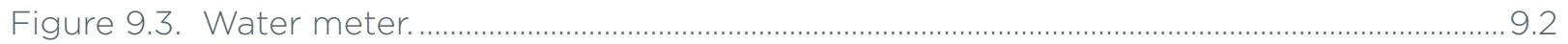

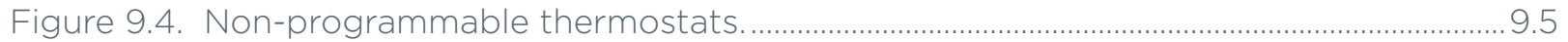

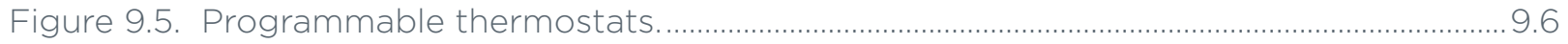

Figure 9.6. Schematic of an economizer and outdoor intake for economizer .............................9.8

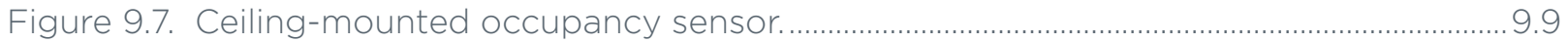

Figure 9. 8. Wall-mounted occupancy sensor. ……………………...............................................

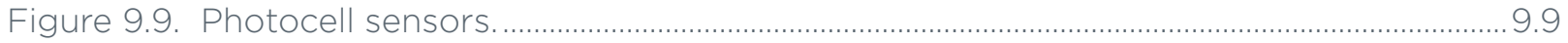

Figure 9.10. Building operation graphic screens................................................................................ 9.10

Figure 9.11. Building operation and maintenance personnel ............................................................ 9.11

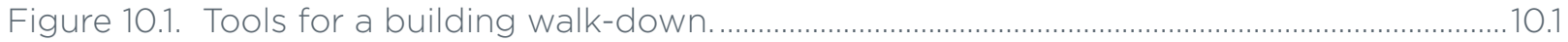

\section{TABLES}

Table 7.1 Summary Table for Lighting Technology Selection. 


\section{ACKNOWLEDGEMENTS}

The authors acknowledge the Building Technologies Office of the U.S. Department of Energy (DOE) Office of Energy Efficiency and Renewable Energy and Washington State Attorney General's Office for the development and demonstration of the re-tuning training. The authors also thank Shalon Brown (Project Manager at DOE) and Linda Sandahl (Project Manager at PNNL) for their thoughtful comments and insights, Michael Perkins for creating many of graphics used in the book, Sue Arey and Susan Ennor for editorial support, Shannon Colson for formatting the content as a handbook. Finally, the authors thank a number of external reviewers who provided valuable comments: David Cranmer, John Lord, Michael Bobker, Susan Hunter, Mike Hatley, and Parhum Delgoshaei. 
Small Commercial Building Re-tuning: A Primer 


\subsection{INTRODUCTION}

Energy costs typically make up a significant portion of overall building operation expenses, yet between 10 and $30 \%$ of the energy used in commercial buildings is wasted because of improper and inefficient operations. To help building owners, building managers, and their operations staff address this issue, the U.S. Department of Energy (DOE) has developed a

building re-tuning training curriculum to identify inefficient operations, detect energy savings opportunities, and implement improvements. Re-tuning is a systematic process of identifying and correcting operational problems at no or low cost. Re-tuning instructional materials have been developed for two general categories of commercial buildings: (1) large commercial buildings with building automation systems (BASs) and (2) small- to medium-sized² commercial buildings without BASs.

Because the re-tuning training for buildings without BASs is intended for a diverse audience, some participants may need additional background information to understand concepts presented in the training. This document provides background information specifically geared toward small- to medium-sized commercial building operations. It introduces basic building energy terminology associated with building energy use to "prime" targeted participants to get the most out of the building re-tuning training. The intent is for participants who are less familiar with the concepts to review this material before taking the building re-tuning training class.

\footnotetext{
'Typically commercial buildings greater than 100,000 square feet have building automation systems
}

${ }^{2}$ Typically less than 50,000 square feet but up to 100,000 square feet. 
Small Commercial Building Re-tuning: A Primer 


\subsection{HOW TO USE THE PRIMER}

This primer is organized by the following building topic areas:

- building exterior (e.g., roofs, walls, windows, and doors)

- building interior

- heating, ventilation, and air-conditioning (HVAC) systems

- lighting

- water-heating systems

- meters

- sensors and controls

- re-tuning tools.

Readers can focus on individual topic areas in which they need additional background information or read the primer as a whole.

The appendix of the primer include a glossary of terms to which terms in bold in the narrative are linked and an appendix, which provides more detail on HVAC system terms, definitions, and efficiency measurements. The glossary is a comprehensive list of building energy-related terms related to all parts of the building.

Various energy-related components of a building are integrated and interact with one another, so one topic area in this primer may reference another. To help readers navigate the document electronically, terms in bold are linked to the glossary, while topic cross references are highlighted in bold green font for easy click-to access to the related material.

For reader convenience, a summary "nutshell" recap of the topic is included at the end of each section. These summaries are intended to capture the fundamental "take away" concepts to be gleaned from reading each section.

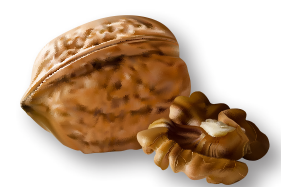


Small Commercial Building Re-tuning: A Primer 


\subsection{INTRODUCTION TO BUILDING ENERGY}

At the most basic level, buildings represent our attempt to control our environment, allowing us to work and live more comfortably. Buildings provide protection from the elements and improve occupants' comfort, health, and safety. While the building structure itself has not traditionally used (or produced) energy, the structure and the interior components (such as lighting and equipment) all interact with each other, influencing the amount of energy the building uses as a whole. The outside environment interacts with the building envelope, which affects the amount of energy needed to maintain desired indoor environmental conditions. The building envelope responds to the outside environment, transferring heat to the colder side of the wall, or allowing natural light to come into the building.
Building equipment responds to these outside environmental conditions according to preset interior controls, incorporating other internal considerations such as occupancy levels and the needs of processrelated equipment (e.g., computers or electronic equipment).

To ensure that these separate but interdependent components work together efficiently, the operation of the building should be actively managed. Building operations provides stewardship and maintenance of the building, including the day-to-day operation and occupancy schedules, to ensure the comfort, health, and safety of those who work in the building. Figure 3.1 illustrates the various components of a commercial building.

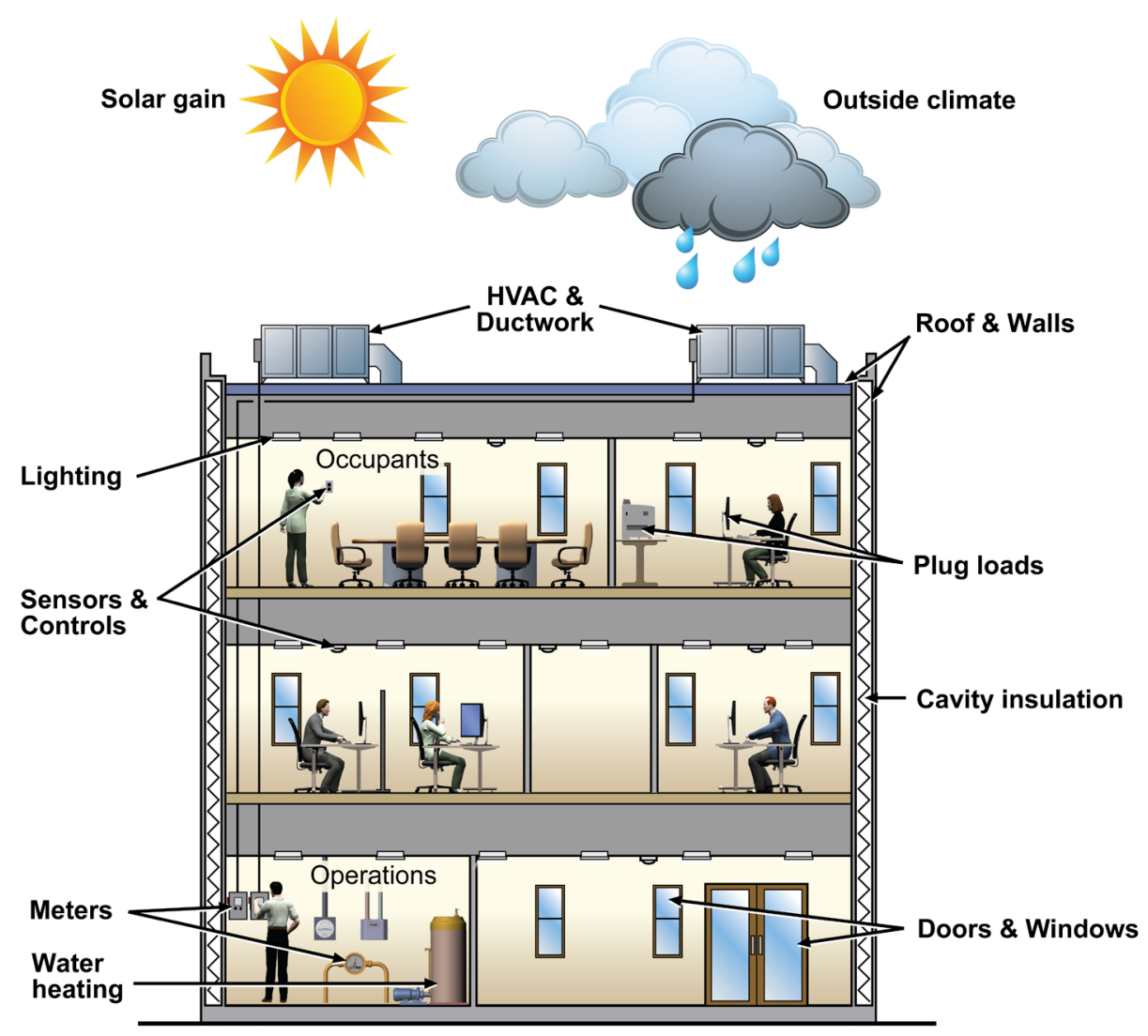

Figure 3.1. Commercial building components. 


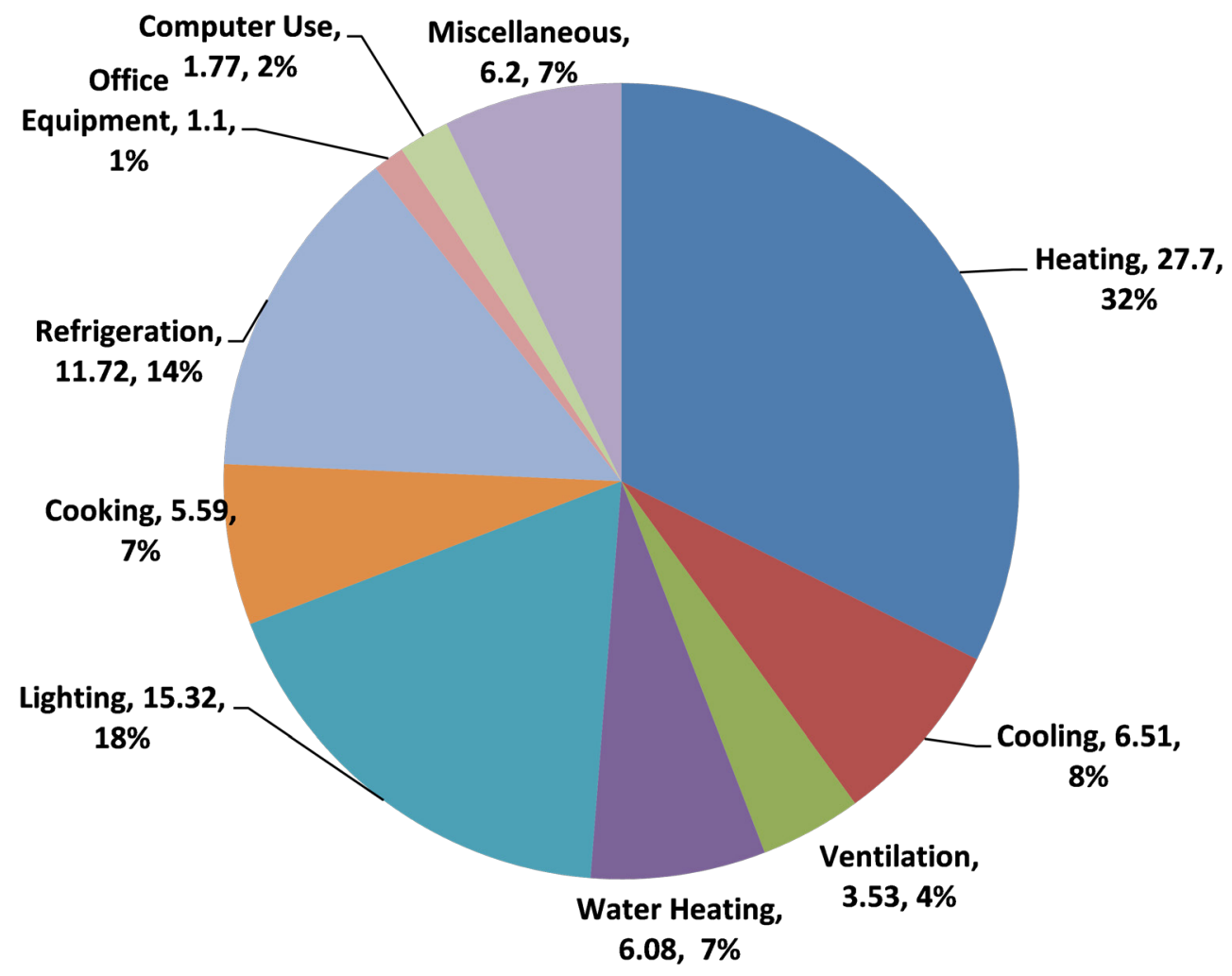

Figure 3.2. Small commercial building (less than 25,000 square feet) energy consumption breakdown (kBtu/square foot/year) and percent of total consumption.

Buildings account for about $40 \%$ of all energy use in the United States; residential buildings and commercial buildings account for about $22 \%$ and $18 \%$ of energy use, respectively. Within commercial buildings, lighting, space heating, and space cooling consume the most energy (see Figure 3.2). ${ }^{3}$

To gain some perspective about how a building uses energy, it is useful to understand how some of the basic features of a building may affect building energy use. The size and shape of a building, the materials with which it is constructed, as well as its directional orientation can affect a building's energy needs in terms of heating, cooling, and lighting. Once a building is built, little can be done to modify its size, shape, or orientation, but a basic understanding of these factors and their impact on building energy use can be used to manage building operations and maintenance. Here are some basic energy implications stemming from a building's size, shape, and orientation:

- The building perimeter tends to have a greater amount of air infiltration from the outdoors as a result of exposure of the exterior walls and roof to the outdoors; thus, there is usually a greater impact from heat gain in the summer months and heat loss in the winter months in these "perimeter zones." Meanwhile, the core of a building (center of the building not

${ }^{3}$ Data splits derived from U.S. Energy Information Administration's "Commercial Buildings Energy Consumption Survey (CBECS) 2003," query of buildings equal to or less than 25,000 square feet. Database available online at: $\boldsymbol{h t t \boldsymbol { p } : / / \boldsymbol { w } \boldsymbol { w } \boldsymbol { w } .}$ eia.gov/consumption/commercial/ Accessed on August 2013. 
next to the outer walls or roof), relative to the perimeter, is typically more affected

by internal heat gains (also referred to as internal gains) from sources such as lights, computers, printers, and people.

- In general, buildings that are more compact are more efficient to heat and cool (and experience less infiltration) than less compact (e.g., high ceiling) buildings.

- Solar heat gain through windows on the south, east, and west sides of a building can affect energy requirements by reducing heating requirements and increasing cooling requirements for perimeter spaces. The balance of these two factors depends on the latitude and climate as well as exterior features that may provide shading to the windows.

The window-to-wall ratio, as well as the quality of the windows, also determine how much solar heat gain affects conditions within the building.

- The side(s) of the building that are exposed to prevailing winds may undergo more wear and tear (because of wind and moisture from rain and snow), which affects the integrity of the exterior wall(s). The entire roof is generally exposed to sun, wind, and moisture for a significant amount of time and therefore undergoes more wear and tear.

- Materials and workmanship used in the building structure itself influence heat transfer and thermal storage, which affect heating and cooling loads. For example, a building built of concrete block has greater thermal mass than a wood-frame building. This means the concrete building can absorb or release heat to the environment with a smaller resulting change in temperature in the building.

In addition to the building structure, energy use is affected by building operation. Building operation is affected by the types of building systems and equipment installed within the building; the control settings for the building's heating, ventilation, and air-conditioning (HVAC) system; the scheduled occupancy of the building; and the maintenance of the building systems and equipment. The energy implications resulting from building operation include the following:

- Equipment types, such as space conditioning or lighting, often have multiple options, including energy-efficient options, available to serve a building's needs. More specialized equipment types, such as electronic equipment, may have only a few options available.

- Some types of buildings cannot tolerate power outages and, therefore, have backup power supplies and redundant systems in place to ensure uninterrupted operation.

- Space conditioning and lighting loads have greater energy requirements during periods when a building is occupied, and sensors and controls could be installed and configured to efficiently reduce energy use during periods of low- or nooccupancy.

- Heating and cooling equipment can be installed to serve single or multiple zones. Zoning allows other factors, such as internal gains or solar heat gain, to be taken into consideration so that each space (with similar heating or cooling loads) is conditioned to maintain comfort at a more energy-efficient level.

Building energy use is also influenced by interactions between end uses. For example, some types of equipment (such as computers or lights) produce heat as a byproduct of their operation. These internal heat gains contribute to the amount of overall heating or cooling needed for the building. Changes to these types of equipment can cause changes in the heating and cooling loads. Energy-efficient lights, for example, produce less heat, causing an increase in 
Small Commercial Building Re-tuning: A Primer

the heating load and/or a reduction in the cooling load. The use of sensors and controls to regulate equipment schedules may also affect heating and cooling loads. Significant changes in occupancy also affect the heating and cooling loads, because people produce heat and use energy differently. 


\subsection{BUILDING EXTERIOR}

The exterior structure of a building-also known as the building envelope or building shell-includes the walls, roof, windows, doors, floors, and cladding or protective siding. Because the building shell is the interface between the interior of the building and the outdoor environment, it serves as a thermal barrier and plays an important role in determining the amount of energy necessary to establish and maintain comfortable conditions inside the building relative to the outside environment.

\subsection{Roofs, Walls, and Insulation}

Roofs and walls can be made using various materials and processes, and the way in which they are constructed and coated affects the heating and cooling requirements of a building. Heat will naturally flow from warmer to cooler spaces. Insulation is often added to provide additional resistance to the flow of heat through the walls and roofs. Insulation can come in the form of an insulating blanket, insulating concrete forms, spray foam, rigid foam, or natural fiber insulation. The effectiveness of the insulation in impeding heat flow is defined by its "R-value"; higher R-values represent higher levels of insulation and thus more resistance to heat flow. The materials used and manner in which a roof or wall is constructed in combination with any added insulation determine the overall insulating characteristics of a roof and wall. Materials like drywall and concrete layers add some additional levels of insulating value, while wall studs and other gaps in installed insulation can decrease a wall's overall insulating value. Walls and roofs are evaluated as a whole, based on the amount of heat transfer through them. Heat transfer is measured in terms of a $U$-factor, which is defined as the heat (in British thermal units per hour $[\mathbf{B t u} / \mathrm{hr}]$ ) that flows through 1 square foot of wall or roof for each degree (Fahrenheit) of difference between the indoor and outdoor temperatures. Lower U-factors represent lower overall heat transfer, which is enabled by thicker insulation layers and/or more effective installation of the insulation.

Commercial building roofs are typically constructed in one of three ways: the "flat" roofing style, low-sloped metal roofs, or sloped/pitched roofs with shingles (like most residential-style roofs) (see Figure 4.1). Construction of commercial building flat roofs involves added insulation installed above the roof deck (or structural roof support). The outer layer of the flat roof design is typically constructed with a prefabricated membrane (e.g., synthetic rubber or polymer), spray foam, tar and gravel, or "built up" with multiple layers of asphalt, insulation, plywood, and a reflective coating (often referred to as a "built-up" roof design). Although commonly referred to as the "flat roof" design, roofs are rarely completely flat because they are designed to gently slope toward one or (usually) multiple drains so that no standing water remains on the roof. Sometimes HVAC units are placed on top of flat-roofed commercial buildings.

In all cases, the reflective and insulating properties of a roof have energy implications. The outer-most layer of a roof is often made up of an elastic, weather-resistant, and (ideally) reflective coating. The elasticity allows the material to expand and contract without damaging the material. The weatherresistant properties protect the underlying roofing layers from the weather and protect the integrity of the outer-most roof layer from the potentially damaging impact of the sun's rays, rain, hail, etc. The reflective properties of the roof coating reflect visible, infrared, and ultraviolet wavelengths from the sun, which reduces the heat transfer to the surface of the roof. Reflective coatings are 
Figure 4.1. Top: highly reflective white flat roof $f^{4}$ bottom left: low-slope metal roof; and bottom right: shingle roof.

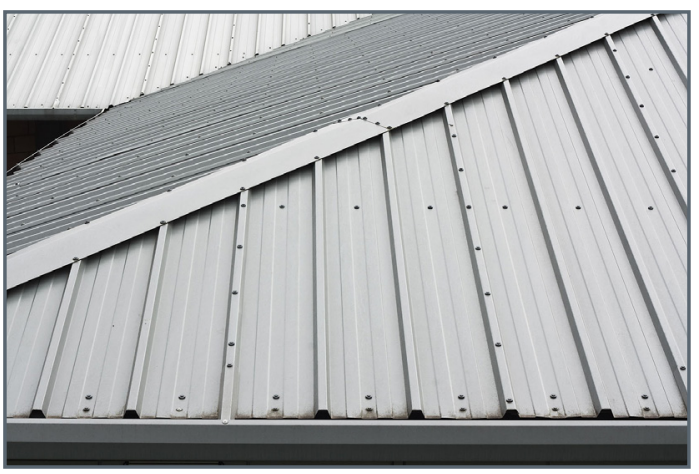

often white or light colors that reflect, and they may sometimes be referred to as "cool roofs." Reflective coatings can significantly reduce heat gains through the roof and, thus, reduce the cooling load of a given building. Despite the energy-saving potential of cool roofs in warm climates, most commercial roofs in these climates do not include highly reflective outer coatings.

A roof curb is a small vertical barrier, typically is used around roof joints and put around anything that sticks out of a roof (see Figure 4.3 and Figure 4.4). Skylights, chimneys, and HVAC systems have roof curbs around them. A roof curb's main function is to channel water away from the seam formed between the roof boards and the opening in the roof so that it does not become saturated with water and become a source of rot, mold, and leaks. Roof curbs are used to support loads

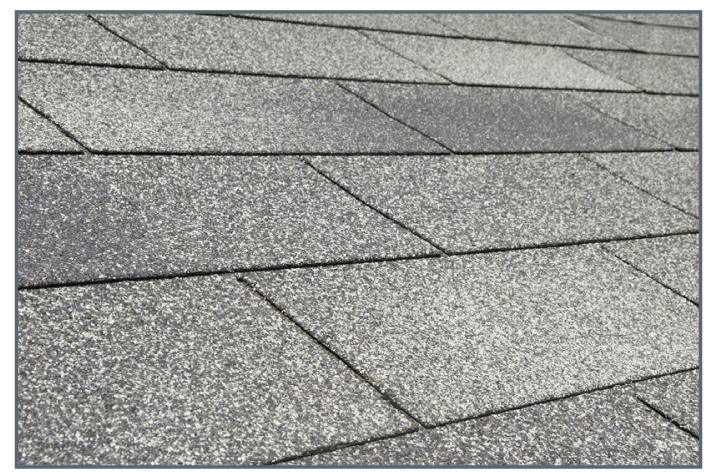

(HVAC systems) that are potentially heavy, so mitigating water penetration that could affect structural integrity is important. Given the likelihood of exposure to high winds and rain/snow, roofs should be insulated on the interior sides and adequately flashed and sealed at all joints and seams to maintain the barrier so water cannot penetrate into the building at the curb structure.

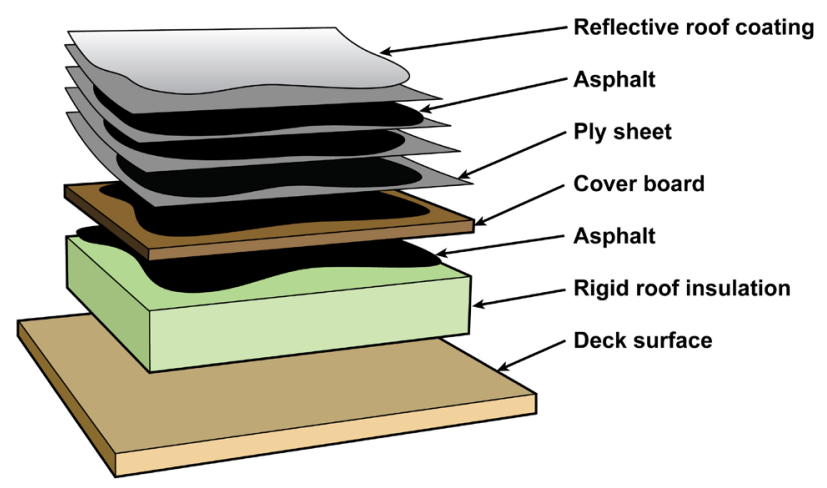

Figure 4.2. Built-up roofing layers 

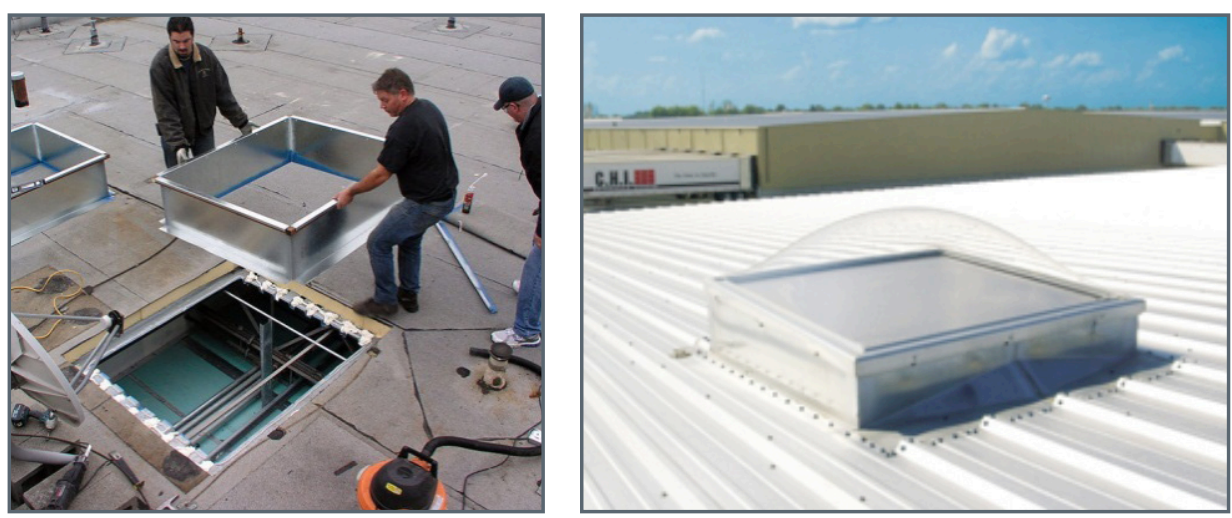

Figure 4.3. Left:

installation of skylight on roof; right: installed roof dome skylight with flashing (Photos courtesy of DayStar).

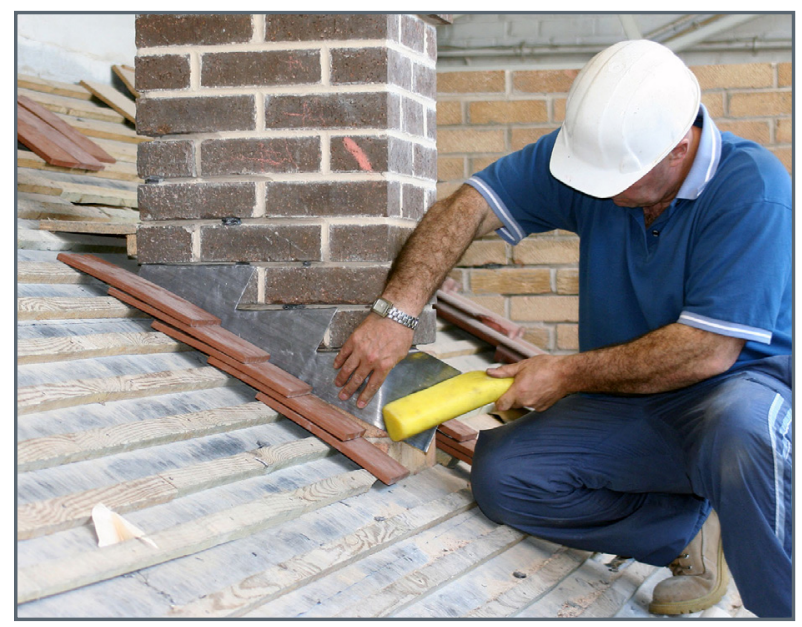

Figure 4.4. Installation of roof curb around a chimney.
Sometimes roofs and pipes in colder climates are equipped with heat traces to keep pipes from freezing and to melt ice that might collect on roofs and in gutters, thereby preventing the roof from draining properly.

A heat trace is a coil of high-resistance wire wrapped around a pipe or gutter, and it is sometimes referred to as electric heat tracing or surface heating. Figure 4.5 shows a diagram of the heat trace resistance wire under a layer of insulation associated with a water pipe (on the left) and a typical control box and heat trace system associated with a roof gutter (on the right).

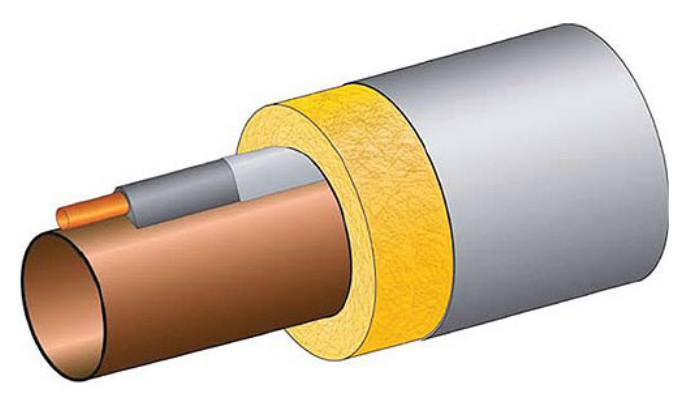

Figure 4.5. Heat trace diagram (left) and typical control box (right).

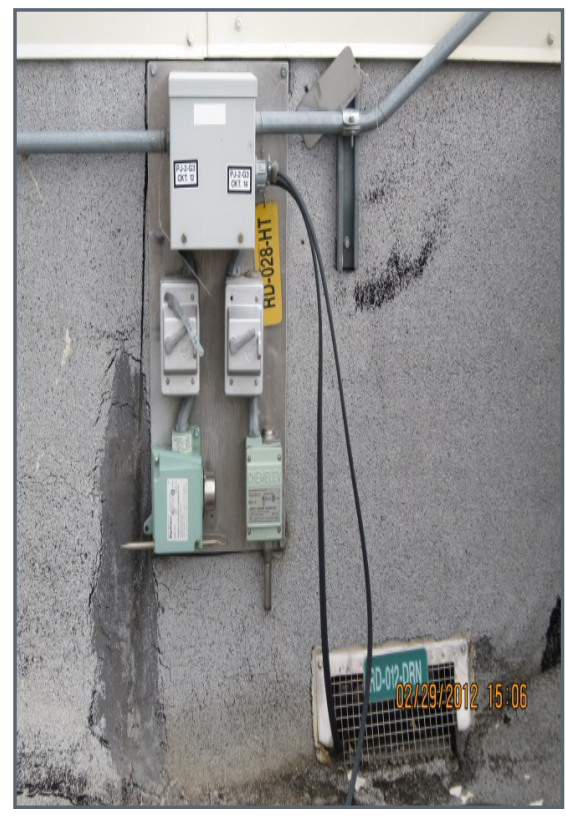




\subsection{Fenestration - Windows, Skylights, and Doors}

The windows, doors, and skylights of

a building are collectively known as

fenestration, which is anywhere in a building where there is an opening in the building envelope connecting the interior of the building with the outside environment. Entryways or doorways can account for a great deal of heat loss or gain in a building, especially in small buildings. Heat is lost (or gained) during the opening and closing of doors, windows, or skylights. Heat can also be lost (or gained) by air seepage between the door frame and the door, along the edges of windows, and around unsealed penetrations (piping, etc.) that pass through walls or roofs. This influx of outdoor air through a window, door, or other wall penetration is called

infiltration; it contributes to interior heat gain in the summer and heat loss in the winter.

When seals between glass panes or between windows, skylights, or doors and their frames are broken or not well sealed, the insulating properties of the window, skylight, or door are reduced (see Figure 4.5). Air leaks through cracks can waste significant amounts of energy and alter the HVAC requirements in a building. Air leaks can cause uncomfortable conditions within the building. Various forms

\section{of caulk and weather stripping are often} used to seal leaks. Door sweeps are often used at the bottom of swinging or revolving doors as a form of weather stripping.
To reduce infiltration losses at entryways, commercial buildings are sometimes designed with recessed entryways to protect against the direct force of prevailing winds.

They may also be designed with an enclosed interior "air lock" space between an entrance door and the main building, which is a double entry, or vestibule. A vestibule creates a tempered zone between the outside elements and the interior of the building, which reduces the amount of warm air lost in the winter and heat gained in the summer. A vestibule also reduces the amount of cold or warm air entering the living space when the interior door is opened. There are a variety of different door types; e.g., solid with insulated core, solid with windows, swinging, revolving, roll-up, etc. Each door type has different insulating properties, or $U$-factors, depending on the material, style, placement, how well the doors are weatherized and sealed and how they are used. Some materials reduce heat flow better than others. Solid wood doors, for example, are typically not as good at reducing heat flow as metal-clad, insulated-core doors. In all cases, a door that is not well-sealed is a source of air leaks and infiltration that can waste energy.

The design and placement of windows and skylights affects the amount of natural light, radiant heat (from the sun), and air leakage that are allowed into a building, all of which affect the HVAC heating and cooling requirements. To improve their
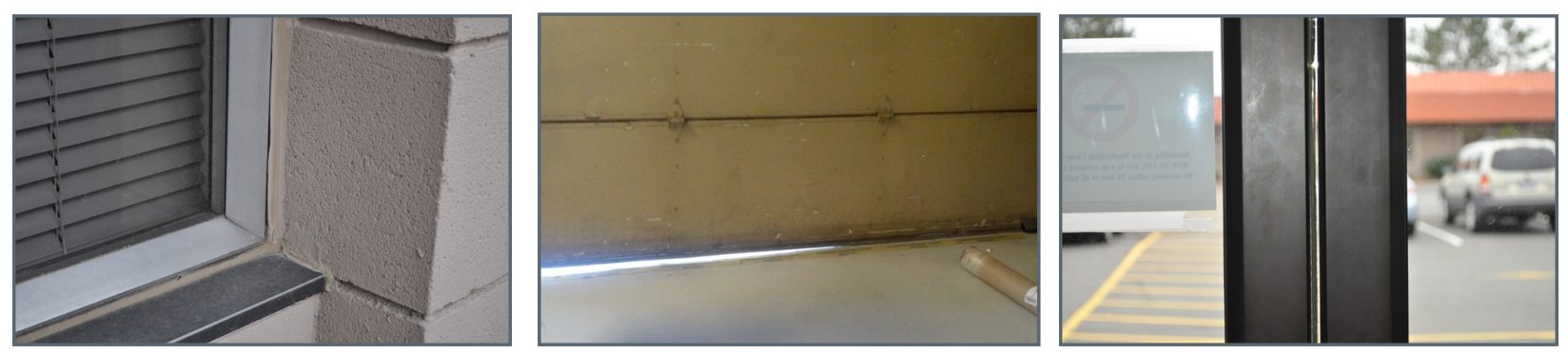

Figure 4.6. Left: caulking on exterior of building between wall and window joints. Middle: bottom of roll-up door is not properly sealed. Right: weather stripping between the double doors is missing on the right door; the lack of a tight seal creates an air gap. 


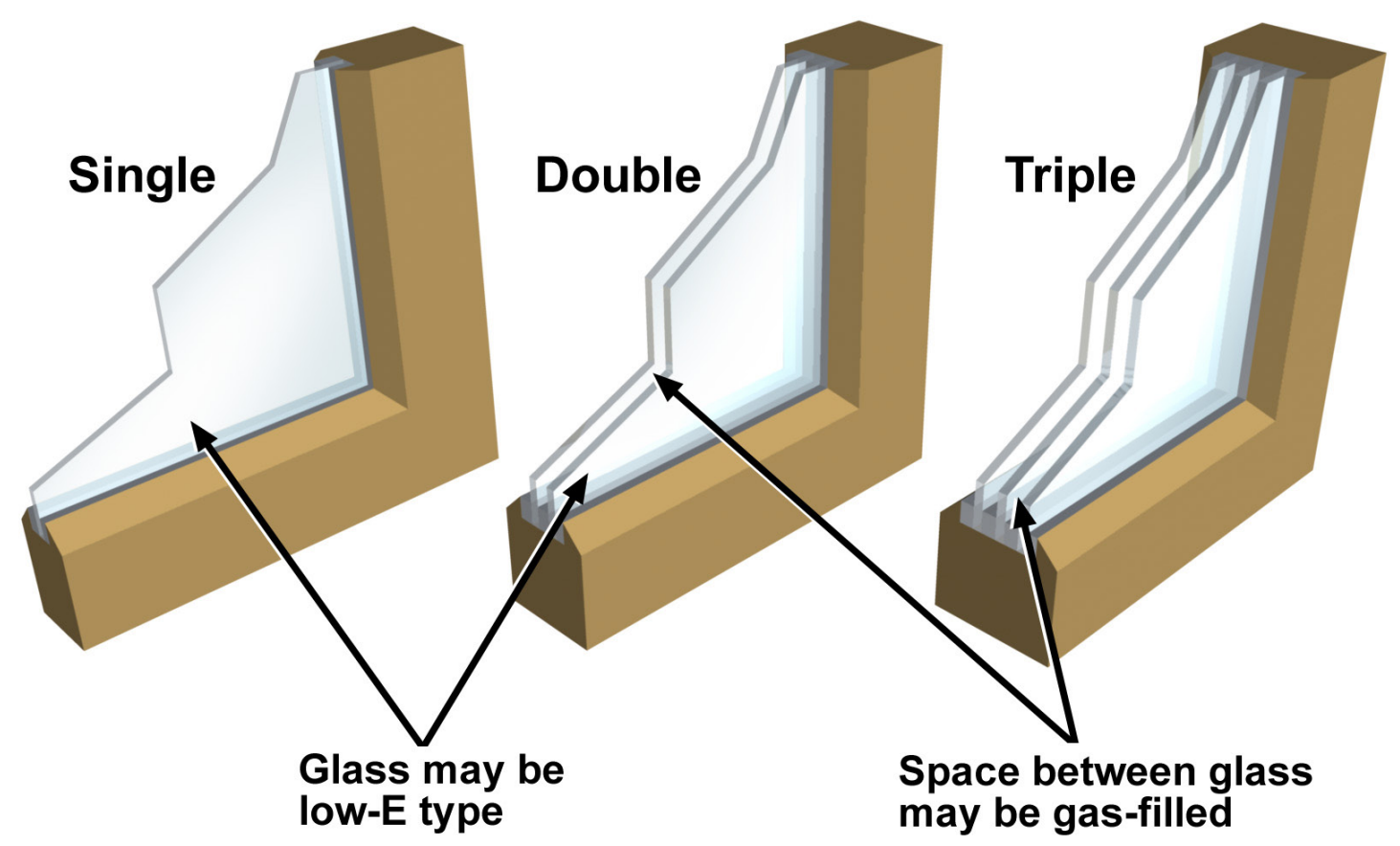

Figure 4.7. Windows can be single-, double- or triple-paned glass.

thermal insulating ability (e.g., the ability to reduce the heat transfer between indoors and outdoors), windows can be made with multiple layers or panes of glass or other films (see Figure 4.7). The space between these glass panes can be filled with an insulating gas (such as argon). Lowemissivity (low-E) coatings, which reduce heat loss from a building by reducing the flow of infrared energy from the building to the outdoors, can be added to the glass to improve the window's insulating characteristics. The more layers of glass, the better insulated the window. Double-pane windows provide three times more resistance to heat flow than single-pane windows, and triple-pane windows offer four times more resistance to heat flow. Because windows have one of the lowest R-values in a building, double-pane windows are usually considered the standard for a well-insulated building. Triple-pane windows are still fairly uncommon in commercial buildings.
You can tell how many panes a window has by looking at where it connects with the frame. If there are no dividers, it is a single-pane window, if there is one divider it is a double-pane window, and if there are two dividers it is a triple-pane window. Windows that you can open and shut are considered "operable windows." Windows that are operable can result in unwanted heat gain and heat loss when left open (even partially open), and they can contribute to security problems (theft/unauthorized access). Inspection of operable windows is encouraged.

Windows can have tinted or reflective coatings to reduce the amount of solar heat energy flowing through them, which can affect the HVAC heating and cooling requirements of the building and reduce unwanted glare. Sometimes, tinted films are added to the windows after they have been installed in a building. Depending on the type and purpose of the film, they can be applied to either the interior or exterior of the window with an adhesive coating. They may require special maintenance. 
Small Commercial Building Re-tuning: A Primer

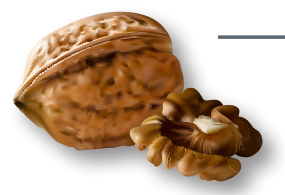

In a nutshell... The reflective

and insulating properties of roofs and walls affect the

HVAC heating and cooling requirements of a building. Because all forms of fenestration (windows, doors, etc.) represent an opening between the inside and outside of a structure, they often are sources of air leaks, which can waste energy. In general, windows are often the least insulated part of a building's exterior and can significantly affect energy usage in the building. 


\subsection{BUILDING INTERIOR}

In terms of energy usage, the thermal load of a building is typically considered the amount of energy demanded by the building to maintain a certain comfort level and to keep it functioning as desired. It is made up of the heating, cooling, lighting, ventilation, water heating, and all other miscellaneous electric loads (i.e., things that are plugged in and drawing power from the building, also referred to as "plug loads").

The amount of energy a building requires varies throughout a day. The manner in which energy demand varies is referred to as the building's load profile. When the variation in energy demand is tracked with a meter, it may also be referred to as a meter profile. A load profile varies according to the overall design of the building, including the amount of conditioned space, as well as the purpose and use of a building. For example, the load profile of a grocery store looks different than the load profile of an elementary school because these buildings have significantly different designs and purposes. The load profile also varies based on the seasons, outdoor conditions and temperature, the time of day and specific daily activities in the building, the day of the week and holiday seasons, or other events that affect building energy use.

In a given day, the hourly energy load varies based on the time of day, building schedule and activities, and outdoor conditions.

Various building energy uses or end uses (e.g., lighting, cooling, ventilation, etc.) have different load profiles as well. Figure 5.1 profiles the hourly electricity consumption by various electricity end uses in a grocery store in Montana on a summer weekday.

Hourly Electric Load Profile Montana Grocery Store (weekday in summer)

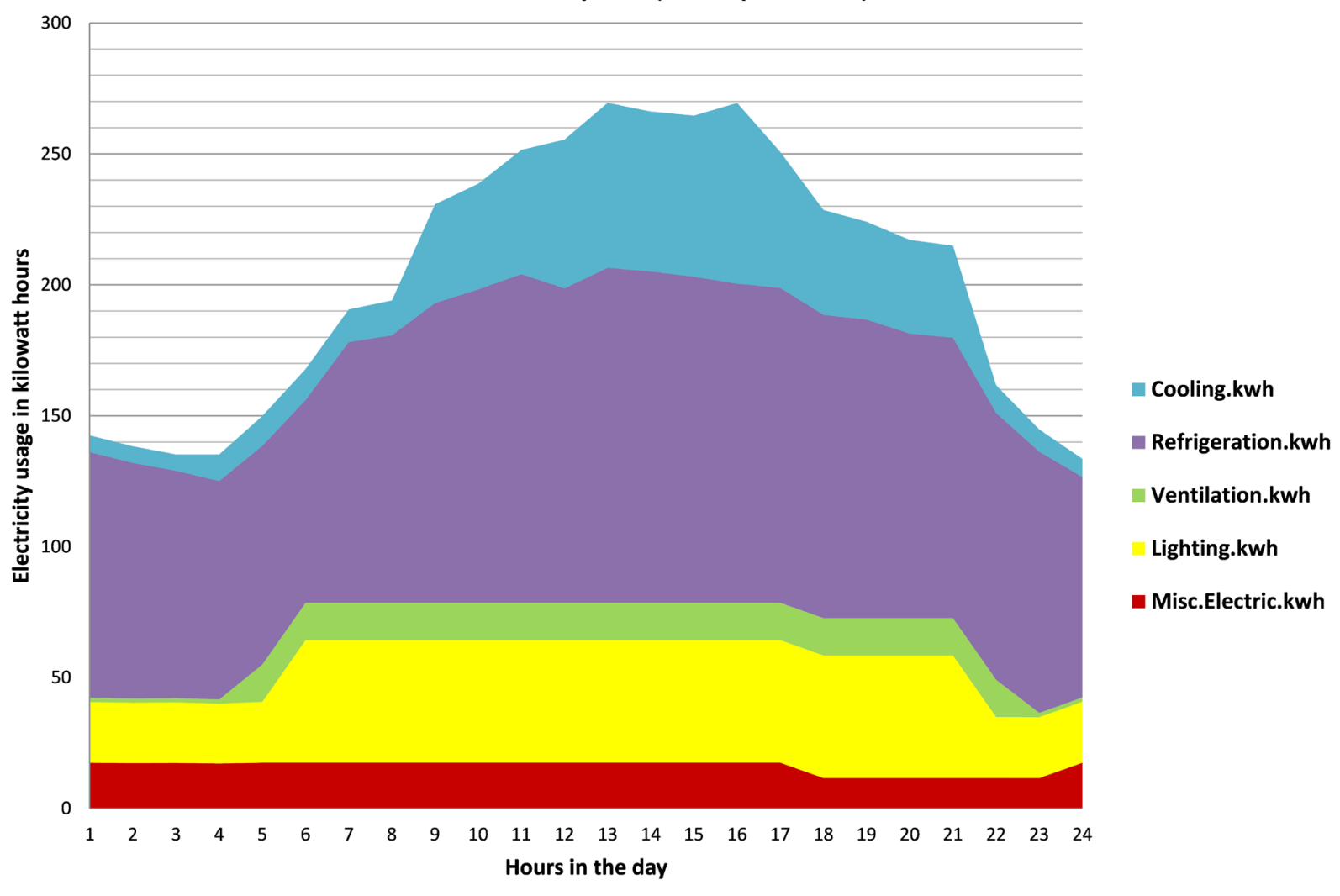

Figure 5.1. Hourly load profile in grocery store (weekday in summer). 
As seen in Figure 5.1, the shape or profile of the electricity use varies by end use. For some of the end uses, such as lighting and miscellaneous electric plug loads, the energy use is relatively stable during business operating hours. When the store opens for business ( 6 a.m.), more lights and equipment are turned on and remain on throughout the operating hours of the store. As the store closes (9-10 p.m.), lights and equipment are turned off and the profile slopes down (although some lights and equipment appear to remain on while the store is closed). For the air-conditioning (cooling) end-use, the electricity use profile also shows an increase in use as the store opens; however, the demands on the cooling system are also influenced by outdoor temperature (hottest during the afternoon) and internal heat gains derived from heat rejection from the refrigerators, freezers, and other equipment, as well as people in the store. These influences cause the cooling end-use profile to peak in the middle of the afternoon and fluctuate more than other end-use profiles.

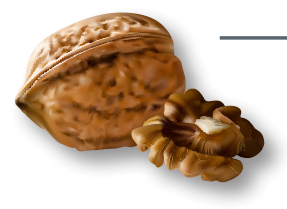

In a nutshell. . . The schedule and activities taking place inside of a building along

with the outdoor conditions largely determine a building's energy load profile. A building with a relative flat load profile (very little variation over a 24-hour period) indicates either a 24-hour operation or improper control, which leads to energy waste. 


\subsection{HEATING, VENTILATION, AND AIR-CONDITIONING}

Collectively, heating, cooling, and ventilation equipment and systems (commonly referred to as HVAC systems) are designed to control the indoor environment. HVAC systems consume significant amounts of energy and are not necessarily designed with energy efficiency in mind. Good design includes three critical missions of the HVAC system in a building:

- Provide comfortable conditions for people working inside the building.

- Remove indoor contaminants, including carbon dioxide and volatile organic compounds (VOCs), and replace contaminated indoor air with fresh outdoor air.

- Protect critical building infrastructure (for example, provide protection against high temperatures for computers and protection against frost/freeze for pipes transporting water).

In some humid climates, a fourth mission is to regulate the humidity to mitigate mold growth.

\subsection{Indoor and Outdoor Conditions}

In terms of human comfort, the indoor air temperature and humidity are the most important variables to be controlled. These two factors combine to determine how much heat and moisture are lost from the skin when a person is indoors. When a person is outdoors, the amount of sunlight and wind speed also play an important role.

We normally think about temperature in terms of the dry-bulb temperature. This is the temperature measured by standard thermometers and reported in weather forecasts. The dry-bulb temperature of the air can be changed without adding or subtracting moisture through a process called sensible heat gain/loss. This can be accomplished, for example, by putting an electric space heater in a room.

The air around us consists of a combination of dry air and water vapor. The amount of water vapor in the air determines its humidity. There are two important measures of humidity: absolute and relative humidity. The absolute humidity is the total amount of moisture in the air (e.g., grams of water per cubic meter of air).

The measure normally used for humidity in weather forecasts and in conventional understanding is relative humidity, which is expressed as a percent and refers to the amount of water vapor in the air as a fraction of the air's moisture-holding capacity at its current temperature. The hotter the air is, the more water it can hold. A reading of 100\% relative humidity means that the air is totally saturated with water vapor and cannot hold any more. When indoors, people tend to feel most comfortable when the relative humidity ranges from around $30 \%$ to $50 \%$.

The American Society of Heating, Refrigeration and Air Conditioning (ASHRAE) engineers develop U.S. standards for establishing comfortable indoor conditions. While comfort conditions vary seasonally, they generally range from $68^{\circ} \mathrm{F}$ to $78^{\circ} \mathrm{F}$ dry-bulb temperature and from 30\% to $75 \%$ relative humidity. Air that is above $60 \%$ relative humidity in indoor environments may be conducive to mold growth on certain surfaces. Therefore, temperatures in the range of $68^{\circ} \mathrm{F}$ to $78^{\circ} \mathrm{F}$ and relative humidity between $30 \%$ and $60 \%$ should be targeted for human comfort. People typically wear more clothing in the winter and less in the 
summer, so for comfort and energy efficiency, temperatures should be kept on the lower end of the scale in the winter and the higher end of the scale in the summer.

Latent heat is the heat required to add moisture to the air through evaporation of moisture. Conversely, heat is released into the air as moisture leaves the air (condenses). The important concepts to understand for building humidity control are that adding moisture to the air with a humidifier either requires an additional heat source (steam humidifier) or pulls heat from the air and lowers the air temperature (misting humidifier). Removing moisture from the air (also called de-humidifying by cooling the air) requires more energy than just cooling the air to the same temperature without removing the moisture.

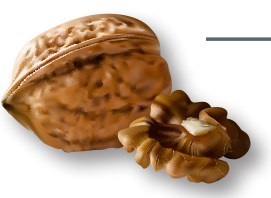

In a nutshell. . Temperature and humidity of indoor versus outdoor air are important drivers of HVAC demands. Indoor temperature and humidity affect occupant comfort and health as well as building infrastructure. The relative humidity and temperature of moist air are linked such that changing one may also change the other. In humid climates, management of indoor temperature and humidity together require more energy than simply managing the indoor temperature.

\subsection{Sources of Indoor Heat, Moisture, and Contaminants}

Heat and moisture can be generated internally or may be transferred to the indoor environment from the outside. Heat and moisture must be removed or they will build up and lead to uncomfortable conditions. Indoor air contaminants must be diluted with fresh outside air through ventilation. Sources of internal heat, moisture, and contaminants added to indoor spaces include the following:

- Lights - Most of the electricity supplied to lighting fixtures ultimately ends up as sensible heat added to the space (even if some of it is converted first to visible light).

- Electric equipment - Similar to lights, devices like computers, copiers, and other electric equipment add sensible heat to indoor spaces. Not all the plug load is converted to indoor heat gains, but a significant portion is.

- People - People give off heat, moisture, and air contaminants like carbon dioxide. Adults at rest indoors typically give off about 50 watts (approximately 160 Btu/ hr) of sensible and latent heat. The heat from two to three people is equivalent to a bank of four linear fluorescent T-8 light bulbs (a typical office light fixture). Indoor spaces are typically designed to be ventilated with 15 to 20 cubic feet per minute (cfm) of outdoor (fresh) air per person.

- Showers, sinks, dishwashers - These components are potential sources of moisture and latent heat gain generated within the building.

- Carpets, paint, pressure-treated wood, and finished furniture - These components are all potential sources of Vocs generated within the building. The ventilation rates should take into account off-gassing from 
these surfaces, or the building can be remodeled with VOC-free paint, furniture, and upholstery.

In addition to these sources of internal heat, moisture, and contaminants, the following sources of heat, moisture, and contaminants may be transferred to the indoor environment from the outdoors:

- Solar gains - Sunlight entering the building from exterior windows may add significant heat to indoor spaces.

- Conduction through the envelope - The envelope of the building includes the windows, walls, and roof that form the exterior boundary between the interior of the building and the outdoors. The magnitude of temperature difference between the indoors and the outdoors governs how much sensible heat is gained or lost from the indoor space. Sunlight absorbed on exterior surfaces also affects this temperature difference. Insulation is the primary means of reducing conduction through the envelope.

- Ventilation air - Air that is intentionally brought in to remove indoor air contaminants may also introduce air that is hot, cold, moist, or dry relative to the indoor conditions. When this is the case, ventilation air may add or remove sensible and latent heat. If this occurs in climate zones dominated by high humidity (moisture), excess ventilation air can be problematic. This can be handled in one of two ways: 1) operate the HVAC systems in a way that removes excess moisture or 2) minimize the amount of ventilation air to meet code or other requirements. When ventilation air exceeds what is required (i.e., is not controlled properly) additional energy may be required to heat the air, cool the air, and remove moisture from the air.
- Infiltration of outdoor air - Additional outdoor air enters all buildings passively. This infiltration is driven by small pressure differentials between the indoors and outdoors. Air may enter the building through small cracks in exterior doors/ windows and through penetrations like electrical outlets and HVAC system vents and dampers. Infiltration can be a major source of sensible and latent heat gain in interior spaces. Infiltration can be a major source of contaminants derived from airborne dust and fumes that otherwise would be filtered at the main air delivery systems, prior to being introduced into the building.

For a given space inside a building, if the sum of the various loads is positive it implies a heat gain and is called the cooling load; if on the other hand the sum is negative it implies a heat loss or the heating load The HVAC system is responsible for adding as much heat as is being removed from the various internal and external sources, when the space is at its heating set point (minimum temperature). Conversely, the HVAC system is responsible for removing as much heat as is being added when the space is at its cooling set point (maximum temperature). Relative humidity set points may put additional demands (more load) on the heating and cooling systems. A building is said to be at its balance point when the heating and cooling loads are both zero. This typically occurs for residential buildings when the outdoor temperature is around $65^{\circ} \mathrm{F}$ and for commercial buildings when it is around $55^{\circ} \mathrm{F}$ (but the balance point is very buildingspecific). The balance point depends on the quality, integrity, and design of the envelope and the amount of internal heat gain. For buildings with larger footprints, there may be no balance point because some interior spaces are isolated (far away) from the envelope and always have a positive cooling load when the building is occupied. 
In a nutshell. .. The function

of the HVAC system is to balance heat and moisture loads to maintain comfortable indoor conditions and to remove air contaminants from the indoor environment. Heat and moisture loads are generated through internal gains inside the building as well as the exchange of external heat and moisture loads with the outdoors. Indoor air contaminants are generated indoors and require ventilation with outdoor air.

\subsection{Zoning}

As discussed with respect to the balance point, the proximity of different internal spaces to the envelope and differing levels of internal gains within those spaces can create areas that have vastly different heating or cooling loads. These different and at times

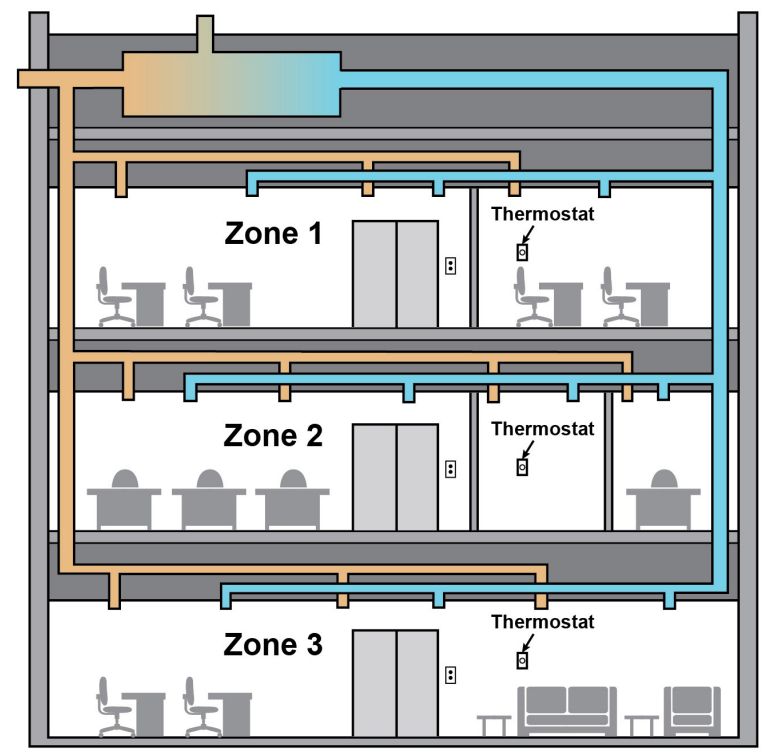

competing demands are handled by zoning Zoning breaks a building into regions that are controlled separately by the HVAC system.

While larger buildings typically have individual HVAC systems that can meet the unique demands of multiple zones at once, smaller buildings typically handle zoning by assigning one packaged rooftop unit to each zone.

For example, the HVAC system in the building on the left in Figure 6.1 has three zones, while the building on the right is not zoned. In the former case, each floor (zone) has its own thermostat, its own temperature settings, and its own air distribution unit. This way each floor can be kept at a separate, automatically controlled temperature. In the latter case all three floors receive the same amount of cooling or heating. ${ }^{5}$ This approach can cause one floor to be heated correctly, while the other floors become over-heated or under-heated. The lack of zoning can cause discomfort and energy inefficiency.

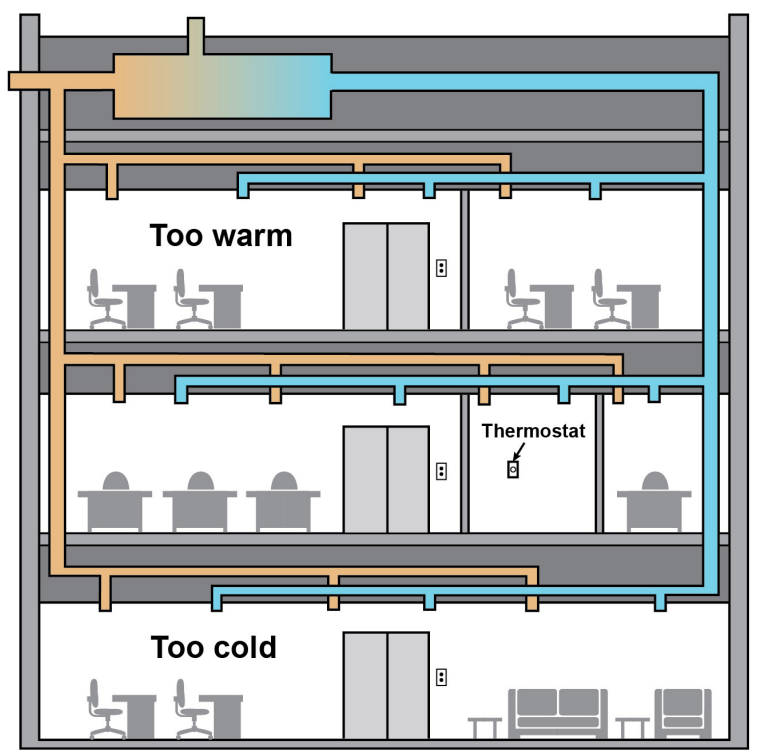

Figure 6.1. The building on left is divided into three HVAC zones. The building on right does not include zones.

${ }^{5}$ The example shows a multistoried building with each floor being a zone. In a single storied building, the zones can be adjacent to each other. 
In a nutshell. . . A multi-zone

approach to HVAC system design allows for better control of indoor conditions throughout the building.

\subsection{HVAC and Air Distribution Systems}

Most HVAC systems are composed of the

following four basic structures/components, which are described below:

- air distribution unit

- outdoor air intake/exhaust

- supply air path

- return air path.
Figure 6.2 depicts an overall view of a typical HVAC air system showing the location of each component relative to the building's zones. The return air path pulls air from the zones into a main branch, where some of the air is exhausted and replaced with ventilation air from the outdoor air intake. The air distribution system contains all of the mechanical and serviceable components of the system including the outdoor air intake, filters, heating and cooling coils, and supply air fan. It also includes the interface to the controls (discussed later) to ensure appropriate heating or cooling of the air and maintenance of minimum ventilation rates when the building space is occupied. After being conditioned to appropriate temperatures and/or humidity levels, the air leaves the air handler and enters the supply air path, where it is distributed to the various zones.

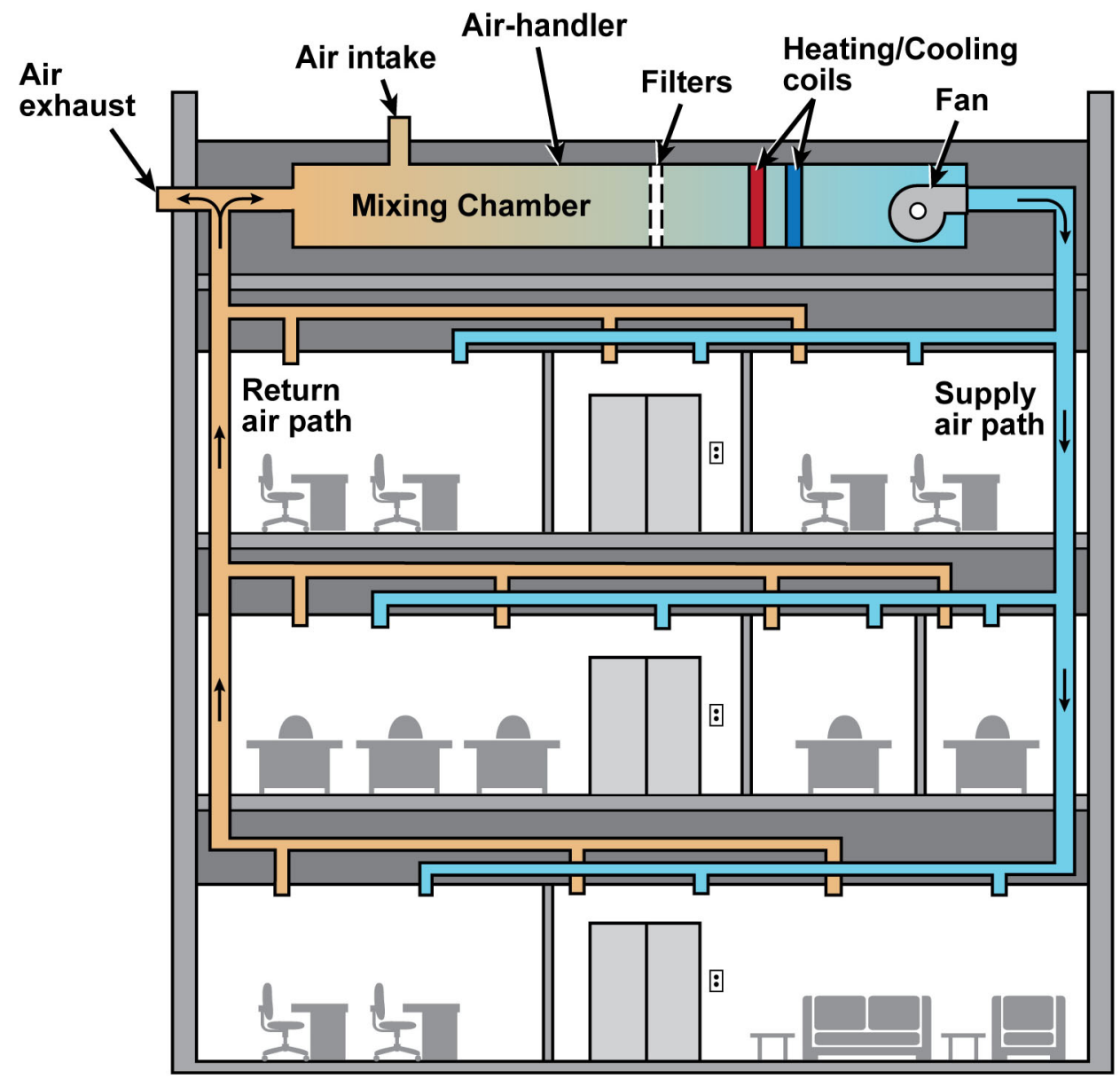

Figure 6.2. Building air distribution system. 


\subsubsection{Air Distribution Unit}

The air distribution unit is the "engine" of the HVAC air system. It houses a supply fan that moves air through the entire system. The fan creates positive air pressure downstream of the fan in the supply air path that drives air into the zones, and negative pressure upstream of the fan that "pulls" air through the return air path and into the air distribution unit. This unit also contains the sources of heating and cooling for the supply air stream-the heating

coils and cooling coils. Most of the heating and cooling sources are heat exchangers, or devices designed to transfer heat from one medium (for example a refrigerant) to another (in this case, air). Heat exchangers are typically tubes in a coiled configuration that maximizes the surface area connection between the hot or cold medium and the air. Finally, the air distribution unit contains air filters that remove dust, particulate debris, allergens, and microbes from the air stream. Filters usually consist of a fine fiber mesh that entraps larger particles, but some filters are electrostatic and work by imparting a charge to contaminant particles and attracting them to a metal filter surface that has an opposing electrical charge. Fiber-based filters need to be replaced when they become loaded or clogged with particulate debris, whereas electrostatic filters need to be cleaned by removing them, rinsing them, and reinstalling them. Filters do not remove gases like carbon dioxide or VOCs. These gases need to be removed by ventilation.

\subsubsection{Outdoor Air Intake/Exhaust}

The outdoor air intake provides a way to mechanically ventilate occupied spaces using the air distribution unit as the engine. Typically, the outdoor air intake is incorporated into the upstream portion of the air distribution unit. The goals and functions of an energy-efficient outdoor air intake are as follows:

- Provide only as much outdoor air as the zones served by the air distribution system require for ventilation when the addition of more outdoor air will increase the heating load or cooling load of the system.

- Provide additional outdoor air beyond ventilation requirements such that cooling loads are minimized when outdoor air temperatures are suitable to do so. This may occur, for example, when the outdoor air temperature is less than 60oF, but internal heat gain has brought the temperature of certain zones above their

\section{cooling set points}

The amount of outdoor air admitted into a building through the outdoor air intake is modulated by the outdoor air dampers The outdoor air damper is part of the

economizer (described later in the section). An economizer damper system consists of variable-pitch damper blades that can be modulated open or closed to adjust the resistance to air flow. A second damper (return air damper) is installed in the return air stream. Closing the return air damper, while opening the outdoor air damper, allows up to $100 \%$ of the air flow in the air distribution unit to come from outdoor air. The economizer dampers are controlled by a control system called an economizer controller to meet the two functions of the outdoor air intake described above.

When outdoor air is introduced into the air distribution system without exhausting an equal amount of indoor (return) air, the building becomes positively pressurized. The indoor (return) air still leaves the building, but slowly, through openings in the envelope and via other dedicated exhaust fans (such as bathroom exhaust and general exhaust 
fans). The supply air fan is forced to work harder because air must be delivered into the building spaces and pulled back (returned) and/or exhausted by the same fan. From the standpoint of the supply air fan, it is similar to blowing into a balloon rather than into a straw. A more ideal system includes a return air/exhaust fan (or relief air) system. The return air/exhaust fan relieves excess building pressure while effectively exhausting indoor air. This system also reduces the work performed by the supply fan by returning air back to the supply fan (via the return air

plenum) or exhausting excess air pressure out the exhaust duct (work that otherwise would need to be performed by the supply fan).

Building exhaust systems include relief air systems in the air distribution units and in the dedicated exhaust fans for bathrooms and other zones. When building exhaust systems exhaust more air than is being brought into the building, negative pressure builds up. Negative building pressurization can cause high rates of infiltration of outdoor air through the building envelope, which can make perimeter areas uncomfortable and increase HVAC energy consumption. The outdoor air intake and exhaust systems can be controlled in a coordinated manner to maintain air balance; i.e., maintain the indoor zones at a neutral or slightly positive pressure.

Many air distribution systems only comprise one fan (the supply fan). These systems are designed to bring in outside air (ventilate) and mix outside air with the indoor (return) air to maintain internal comfort settings without mechanical heating or cooling. When an air distribution system is designed with a return fan, it is designed to return air back to the supply fan (through the return air damper) and to also help exhaust the indoor air to the outside of the building (through the exhaust air damper). See Figure 6.3 for a depiction of this system.

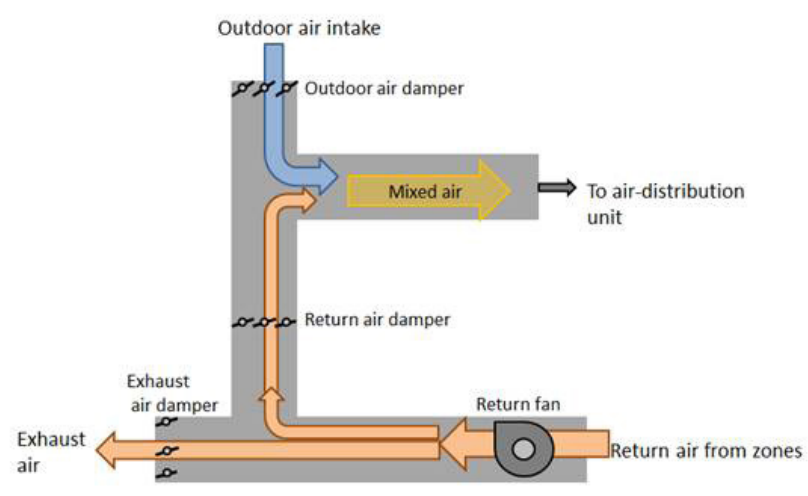

Figure 6.3. Outdoor air intake, exhaust, and mixing box for a system with a return fan.

When air distribution units do not have a return fan they must rely upon one of the following three methods to relieve/exhaust indoor air as more outside air is brought into the building:

1. A return air damper is always located between the return duct and the outdoor air intake. It allows a positive pressure to build inside the building as the return air damper closes and a negative pressure to be induced at the outdoor air intake as the outside air damper opens. Eventually the pressure inside the building increases enough to overcome the envelope resistance and push the indoor air out any connection point between the interior and the exterior of the building. These connection points include broken or missing door seals, broken or missing window seals, exterior outlets, unsealed piping that routes from the interior to the exterior of the building, exterior doors that swing open, etc.

2. Newer air distribution units can be designed with a gravity-weighted backdraft damper that opens automatically to relieve excess interior building pressure. With the advent of buildings that are becoming tighter (at their envelopes), this provision is becoming increasingly necessary. It sometimes helps avoid the "open" door problem described previously. 
3. Newer air distribution units can be designed with automatic relief mechanisms. Such mechanisms can include a powered exhaust fan that automatically activates when the outdoor air damper is more than 50\% open (or as set). The intent is to program the powered exhaust fan to only run when excess building pressure is occurring due to the amount of outside air being introduced. Most powered exhaust fans only run intermittently, while return fans run continuously during the occupied period. This is because powered exhaust fans are located further downstream from where the return fan location is shown (usually after the return duct and next to the exhaust air damper, as shown in Figure 6.3). This means powered exhaust fans can pull the building pressure more negative if the amount of outside air being introduced to the supply fan is not equal to or greater than the air being exhausted.

The return air damper and exhaust air damper help maintain air balance within the air distribution unit when a return air fan is part of the system. Systems with and without a return fan are depicted in Figure 6.3 and Figure 6.4.

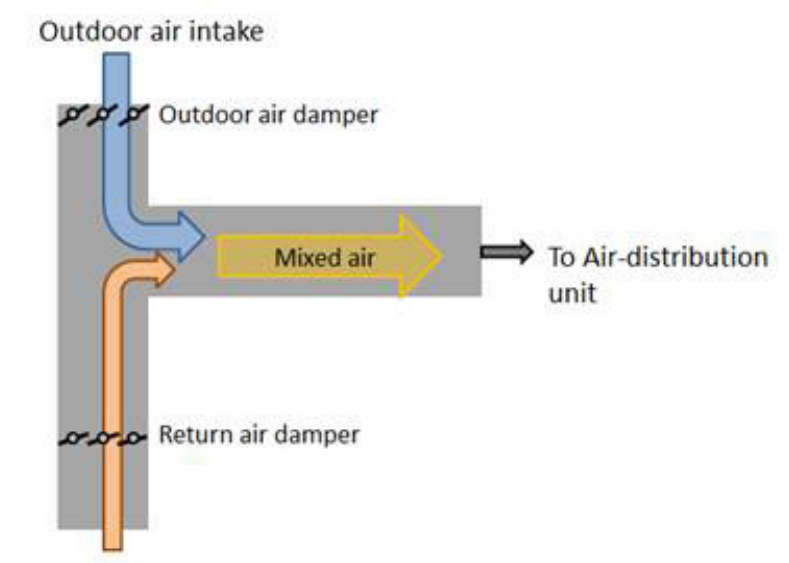

Return air from zones

Figure 6.4. Outdoor air intake and mixing box for a system with no return fan.

\subsubsection{Supply and Return Air Paths}

The supply air and return air paths are similar to the blood vessels of the human body. In the human body, oxygen-poor blood is removed from tissues by small venous capillaries, which consolidate into veins and are drawn into the heart; the oxygen-rich blood is then pumped out via arteries and delivered to the same tissues by arterial capillaries. The air distribution unit is the "heart" of the HVAC air system, and the return and supply paths are its "veins" and "arteries." Ducts, as the "blood vessels" of the air system, are typically made of flexible tubing or sheet metal. Some duct arrangements of various sizes are shown in Figure 6.5.
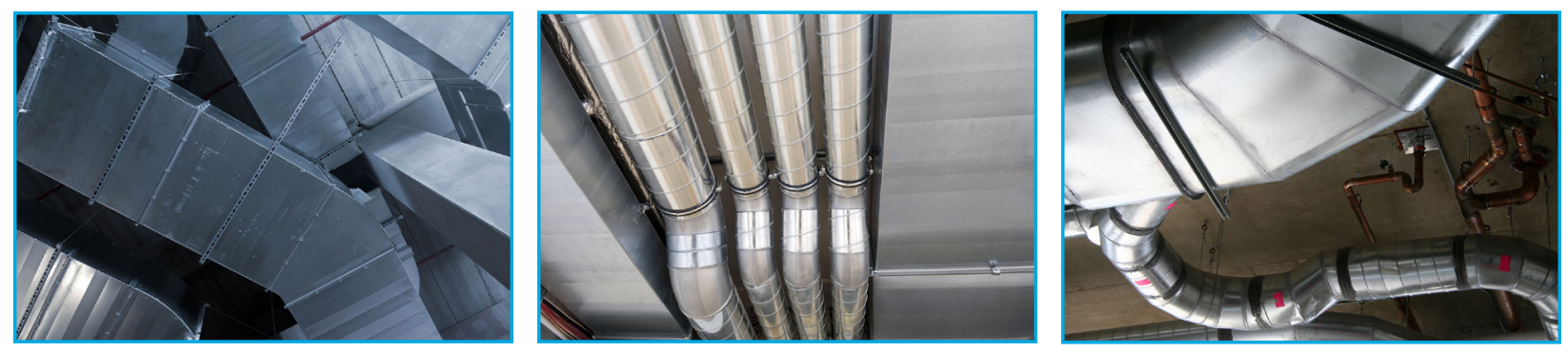

Figure 6.5. Examples of duct arrangements in commercial buildings. 
Small ducts from the zone air return are consolidated into one main return air branch or header. Likewise, a large supply air header is split into supply air branches (typically one per floor) and then split further into small ducts that supply air to a zone air terminal device. Typically, return air ducts are uninsulated because they carry air that is at a comfortable interior temperature. Depending on the length of the ductwork, supply air ducts may need to be insulated to maintain the temperature of the supply air on its way to distant zones. Sometimes, instead of a duct the main return or supply air branch may be an open building space called a plenum, often found between the ceiling of one story and the floor of the next story. Plenums can cause the return or supply air to pick up more heat from light fixtures and may draw in more outdoor air.

\subsubsection{Air Terminal Devices}

A constant air volume system uses a constant speed supply fan and fixed or manual air flow control on air terminal devices. The conditioned air is eventually delivered to the building space through a simple device like a

register or a diffuser in a constant air volume system (see Figure 6.6). This is in contrast to larger buildings, which may have more complex variable air volume (VAV) terminal units with automatic dampers and/or heating coils. A register is a supply air terminal that includes a manual damper that can be used by a zone occupant to adjust supply air flow. A diffuser is an air terminal that spreads out the flow of supply air so that the supply air does not blow forcefully on the occupants and create a wind chill effect.

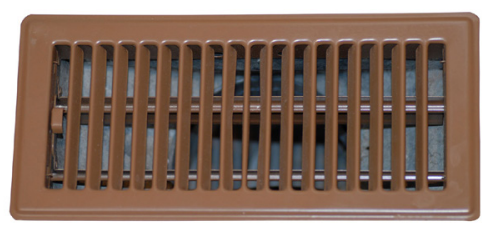

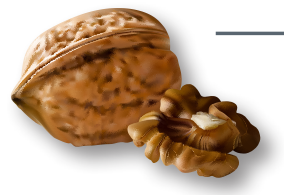

In a nutshell. . . Within the air side of an HVAC system, air leaves the building zones through a return air path. This air is either released out the exhaust section or it is mixed with outdoor air (coming from the outdoor intake) for ventilation and air balancing. This mixed air passes through filters to remove dust particles and microbes. It then passes through heating and cooling coils to warm or cool the air to the desired supply air temperature. A supply fan installed at the outlet of the air distribution unit moves the air through the entire air distribution system network of ducts to individual building zones. At the end of the supply air distribution system, air terminal devices distribute the mixed, filtered, and conditioned air to the individual zones.

\subsection{Cooling System Types}

In commercial buildings, two primary types of equipment are used for air cooling: chillers and direct-expansion (DX) systems. Chillers are typically centralized air-conditioning systems that are not commonly used in smaller commercial buildings; most small commercial buildings use DX rooftop units. A typical chiller works by using the vapor compression refrigerant cycle (described in the next section) to chill water, which is

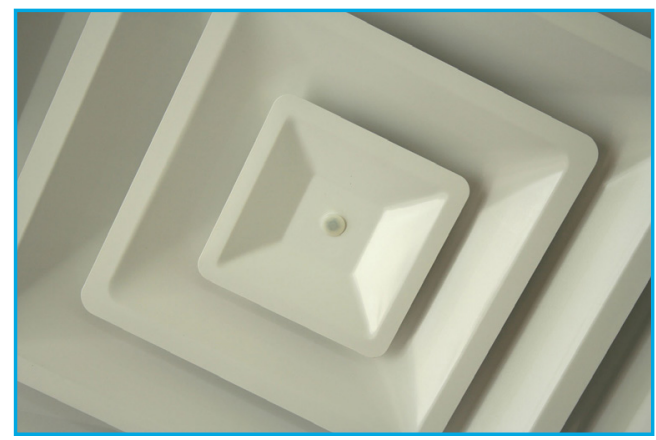

Figure 6.6. Examples of air terminals 
then piped to cooling coils that are part of different HVAC systems scattered throughout the building. Air is then blown over the chilled water cooling coils to provide cool air throughout the building. The DX systems also use the vapor compression refrigeration cycle, but distribute the refrigerant directly to the DX cooling evaporator coils. The "expansion" part of the "DX" acronym refers to the treatment of the refrigerant (an expansion valve reduces the pressure and temperature of the refrigerant liquid) prior to its entering the evaporator. Packaged systems are commonly installed on the rooftop or by the side of a building in one single "package." Such single rooftop units include all components of the refrigeration cycle, the air distribution unit, and the outdoor air intake/exhaust (see Figure 6.7).

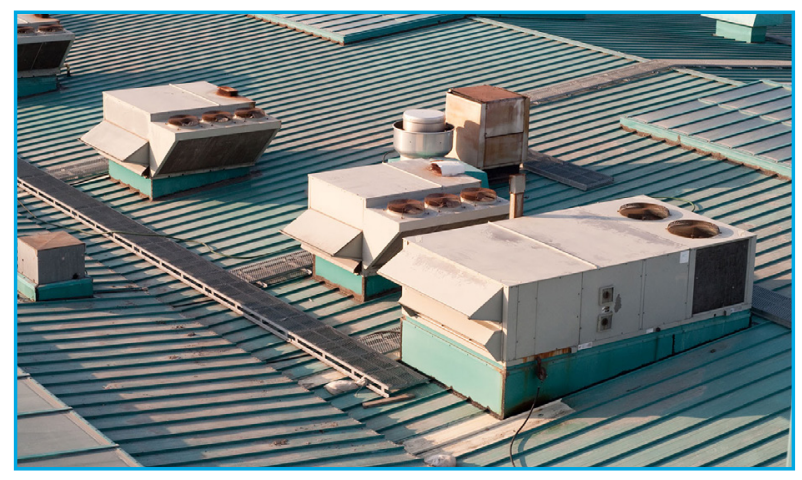

Figure 6.7. Example of several packaged rooftop units.

\section{Split systems have the evaporator coil} and supply fan located inside the building, while the condenser and a condenser fan are located outside (see Figure 6.8). Split systems are typically used in residential buildings, but they are also sometimes used in small commercial buildings.

\subsection{Cooling Equipment}

Air conditioning refers to the process of cooling the air flow in the air distribution unit using a cooling coil.

The cooling coil is one component of a separate sub-system called the vapor compression refrigeration cycle. It is the same cycle and involves the same set of components found in a refrigerator, but it is typically scaled much larger to cool a larger volume of air. The refrigeration cycle is used to pump heat. It is analogous to using a pump to move water up a hill. In the case of the water pump, energy is needed to overcome the force of gravity and move the water from a place of lower potential energy to a place of higher potential energy. The refrigeration cycle works in the same way. It is used to "pump" heat from a lowtemperature source to a high-temperature

sink. In the water pump analogy, gravity is the natural gradient that tends to pull the water to the lowest possible elevation. In the case of the refrigeration cycle, heat naturally flows from a higher-temperature medium to a lower-temperature medium.

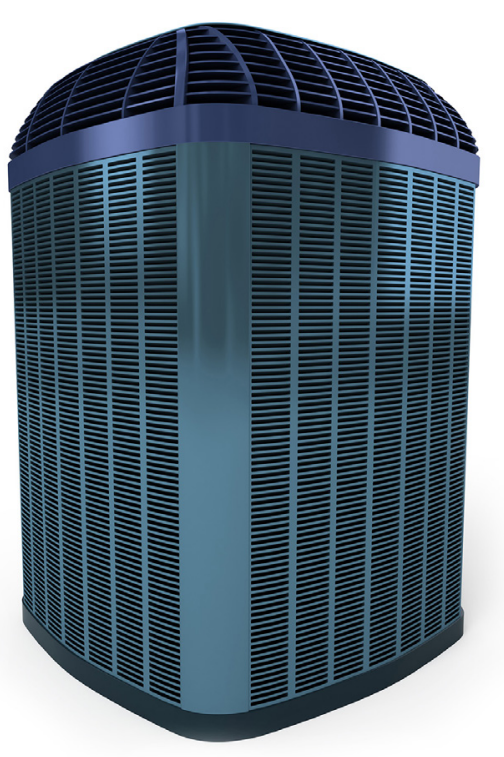

Figure 6.8. A condenser unit for a split system that contains a condenser coil and a vertical fan that moves air through the coil, increasing its capacity for heat rejection. 


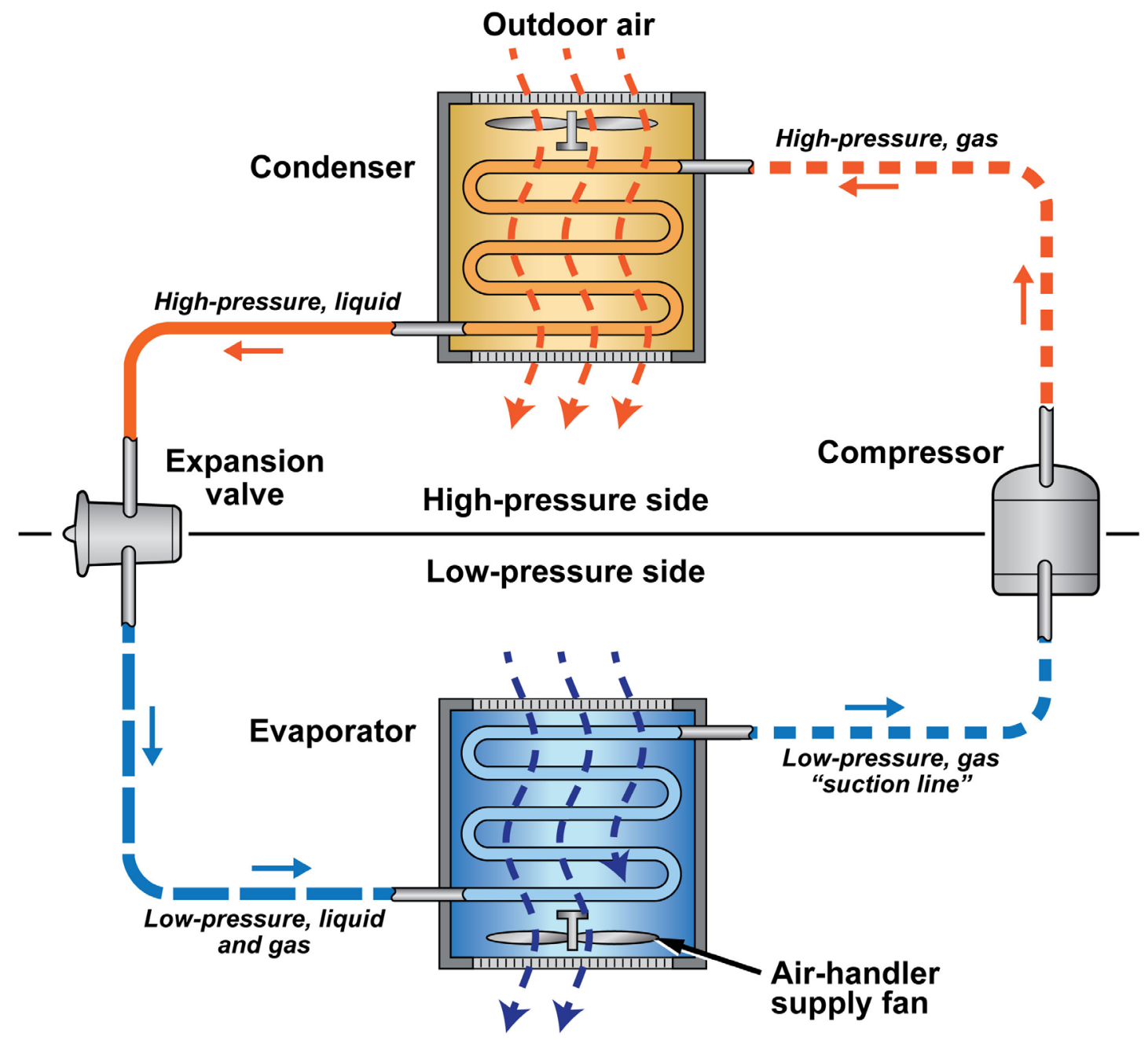

Figure 6.9. Diagram of the refrigeration cycle.

The refrigeration cycle (See Figure 6.9) uses a compressible fluid called the refrigerant or "working fluid." The heat pump segregates the fluid into sections within a cycling loop: a low-pressure section and a high-pressure section. A compressor is used to pump the fluid from the low-pressure section to the high-pressure section by compressing the volume of the fluid. An electrical energy input runs the compressor motor. When a fluid is compressed, its temperature is increased. Therefore, the high-pressure fluid (in the gas phase) at the compressor outlet is also much warmer than the low-pressure fluid at the compressor inlet.
The hot, compressed gas leaves the compressor and flows through a highpressure and high-temperature heat exchanger called the condenser. It is called a condenser because the refrigerant is engineered to go through a phase-change process from gas to liquid. This allows heat to be rejected from the condenser at a constant temperature, which increases the amount of heat that can be rejected. In the case of the air conditioner, the condenser is located outside the building and rejects heat to the ambient environment because the condensing refrigerant is warmer than the ambient outdoor air temperature (even in the summer). 
After heat rejection in the condenser, the refrigerant (now a liquid) flows through a metering device (either a valve or a fixed orifice device) that restricts the flow. Metering devices are like hourglasses; they create a restriction that only allows a controlled amount of fluid to be released through a small opening. As the liquid flows through the opening its pressure drops to match the low-pressure side of the system. (see Figure 6.9 for more details).

Before the refrigerant flows back to the compressor on the low-pressure side of the system, it passes through a lowtemperature, low-pressure heat exchanger called an evaporator. Like the condenser, the evaporator exchanges heat at constant temperature and pressure, evaporating the remainder of the liquid phase of the refrigerant into the gas phase. The evaporator in the air conditioner is the cooling coil located in the air distribution unit. Because the refrigerant is cooler than the conditioned air, heat flows into it, thereby cooling the supply air at the same time.
If the cooling coil is colder than the dew point of the air going over the coil (supply air), water vapor condenses on the surface of the cooling coil. This water drips off the coil surface into a drain pan and the water drains through a drain pipe routed to the outside of the air distribution unit. Because the cooling coil is upstream of the supply fan, the pressure in the air distribution unit is negative near the coil. This means if the drain pipe is dry, air will be drawn in through the drain pipe into the supply air, thereby reducing the effectiveness of the system. To avoid drawing in air through the drain pipe,

a p-trap can be installed that, when filled with water, prevents the backflow of air. It is important to keep the p-trap filled with water in the spring, when it can otherwise dry out because the cooling coil is not being used or humidity is too low to cause it to be filled with condensed water from the coil. During freezing conditions, however, certain p-traps may break if there is too much water in the trap. The diagram in Figure 6.10 shows a p-trap. With this setup, the trap can be kept filled with water by periodically filling through the vent pipe.

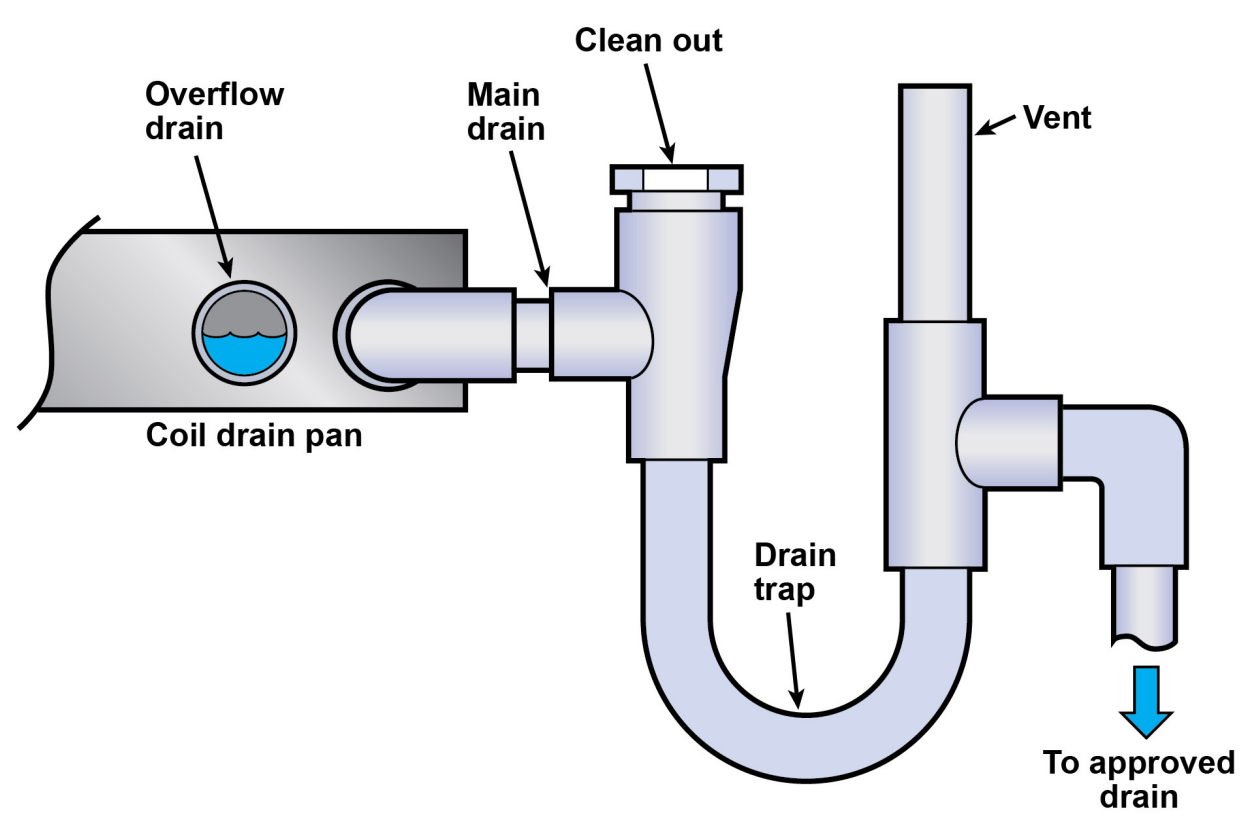

Figure 6.10. Diagram of a typical p-trap. 


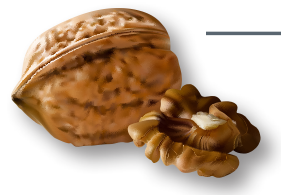

In a nutshell. .. Cooling

equipment in small buildings

uses the vapor compression

refrigeration cycle. This cycle pumps heat out of the building by collecting heat in the cold evaporator and using a compressor to pump it into an outdoor high-temperature heat exchanger called a condenser. Small buildings typically use simple DX cooling equipment, where the cooling coil is used as the evaporator in the refrigeration cycle. DX cooling equipment may have the refrigeration cycle equipment and air handler packaged together in one outdoor unit (usually located on the roof), or it may be a split system design where most of the DX cooling equipment is indoors and the condenser unit is located outside.

\subsection{Heating Equipment}

Various heating equipment, including furnaces, radiant heaters, heat pumps, and boilers, is commonly used in small commercial buildings. The advantages and common applications of each type of equipment and how each functions are described below.

\subsubsection{Furnaces}

Furnaces are the most common type of heating equipment found in small commercial buildings, typically because they have a very low first cost and are compatible with ducted forced-air systems that have already been designed into buildings for cooling. Many packaged air-conditioning units also include a furnace in their airhandler section. There are typically two types of furnaces: gas ${ }^{6}$ and electric. A gas furnace uses a flame to burn natural gas inside a vented chamber. The chamber (see Figure 6.11) is inside the air distribution unit and the air that passes around the chamber is heated. The flame cannot be burned directly in the air distribution unit because the combustion process produces excess carbon dioxide, carbon monoxide, and moisture, which are removed by a separate ducted venting mechanism. Gas furnaces range in efficiency (the fraction of combustion heat transferred to the air stream) from about $80 \%$ to over $90 \%$.

Electric furnaces use electric resistance heating elements (similar to an electric range-top coil) embedded in the air distribution unit to deliver heat directly to the supply air. Electric resistance coils are nearly $100 \%$ efficient at delivering electric energy to the air stream as heat, but because electricity is typically 3 to 5 times more expensive than natural gas per unit of energy, electric resistance furnaces can be significantly more expensive to operate.

${ }^{6}$ In some regions of the country oil furnaces are still used. Although not identical, their operation is similar to that of gas furnaces. 


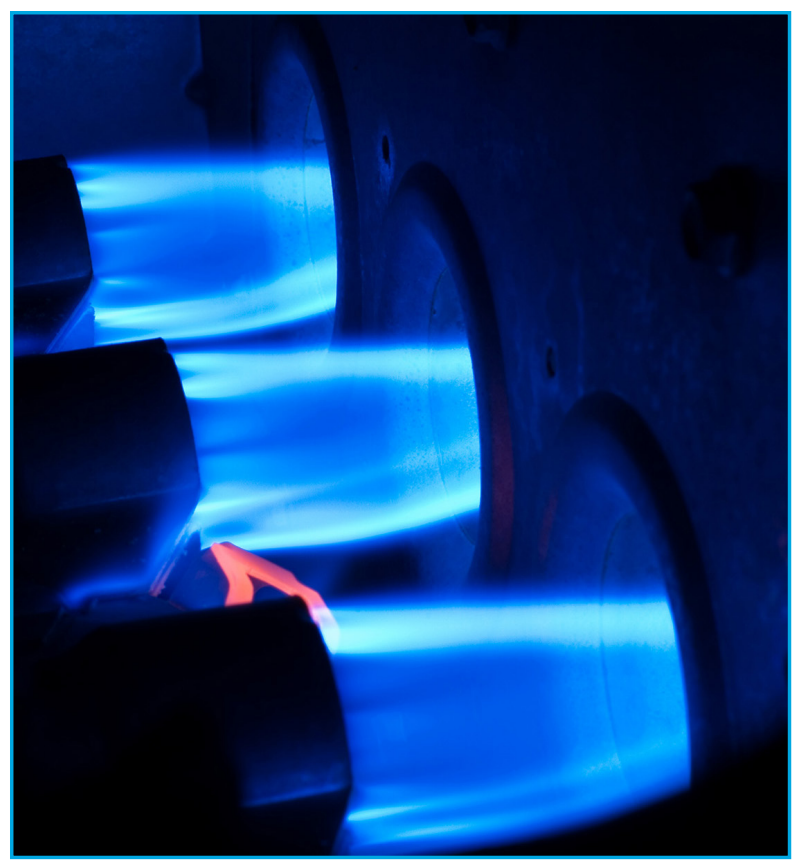

Figure 6.11. Heating chamber inside a gas furnace.

\subsubsection{Radiant Heaters}

Radiant heaters transfer radiant energy from a heat source to a room or object without the use of the central air distribution system. A radiant heater, which contains a heating coil, is placed directly in the zones that require heating (see Figure 6.12). The radiant heater coil is heated with electric resistance, gas, hot water, or steam. Baseboard heaters and radiators can be designed to work with any type of heating coil (electric resistance, hot water, or steam). Some baseboard heaters can also use convection to heat the space served, by including a fan that blows air over the coil and directly into the room to increase the heat transfer capacity of the coil.

Radiant heaters can provide for a more energy-efficient building (when designed correctly) than a furnace because the heat can be delivered only where it is needed. Typically, in the winter, heat is only needed around the perimeter of a building (where heat is lost to the outside), so a typical configuration for radiant heaters is a perimeter baseboard configuration.

One emerging technology that uses radiant heating in some newer energy-efficient building designs is the radiant floor or ceiling panel. It is a structural component of the floor or ceiling that has flexible tubing, typically embedded in a concrete matrix. Heated or cooled water is pumped through the tubing in the floor or ceiling to passively heat or cool the space through radiation. Because the surface area of the "heater" is much larger than a radiator, the ceiling or floor does not have to be substantially warmer or cooler than the space to provide the desired conditioning.
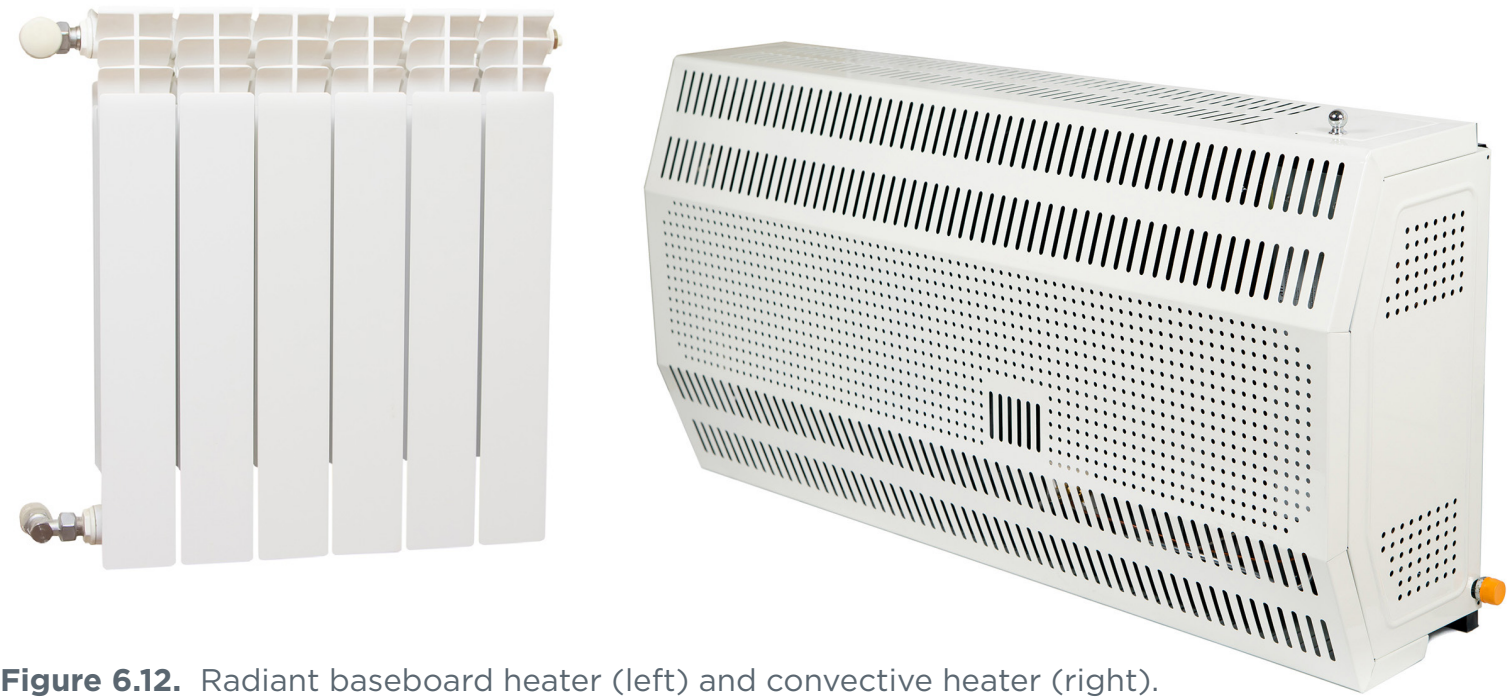

Figure 6.12. Radiant baseboard heater (left) and convective heater (right). 


\subsubsection{Heat Pumps}

A heat pump is a type of an air-conditioning system that uses the vapor compression refrigeration cycle to either heat or cool the air stream. In a heat pump the physical system is the same as an air conditioner, except that a device called a reversing valve sends the refrigerant from the compressor in either direction through the rest of the refrigeration cycle (see Figure 6.13). This means that when heating is required, the heat exchanger in the air distribution unit switches from being the evaporator when cooling is required to being the condenser when heating is required. The outdoor heat exchanger switches from being the condenser when cooling is required to being the evaporator when heating is required.

One limiting factor of air-to-air heat pumps is that they perform less efficiently at colder outdoor air temperatures. At colder outdoor air temperatures the coefficient of performance (COP) drops. This reduces the amount of heat that can be delivered by the heating coil (reduced heating capacity). As a result, the air distribution unit in a heat pump system generally includes an additional gas or electric heating coil called an auxiliary heating coil. If it gets too cold, the heat pump compressor shuts off for protection, and the auxiliary heating coil is employed to meet the heating load demand on the system.

\subsubsection{Boilers}

Boilers are typically the primary heating equipment in large commercial buildings. They are less common in small commercial buildings, but they may be more prevalent in some areas of the country. A boiler typically uses a fuel like natural gas, propane, or fuel oil in a vented combustion chamber, much like a furnace. Whereas a furnace directly transfers the combustion heat to the supply

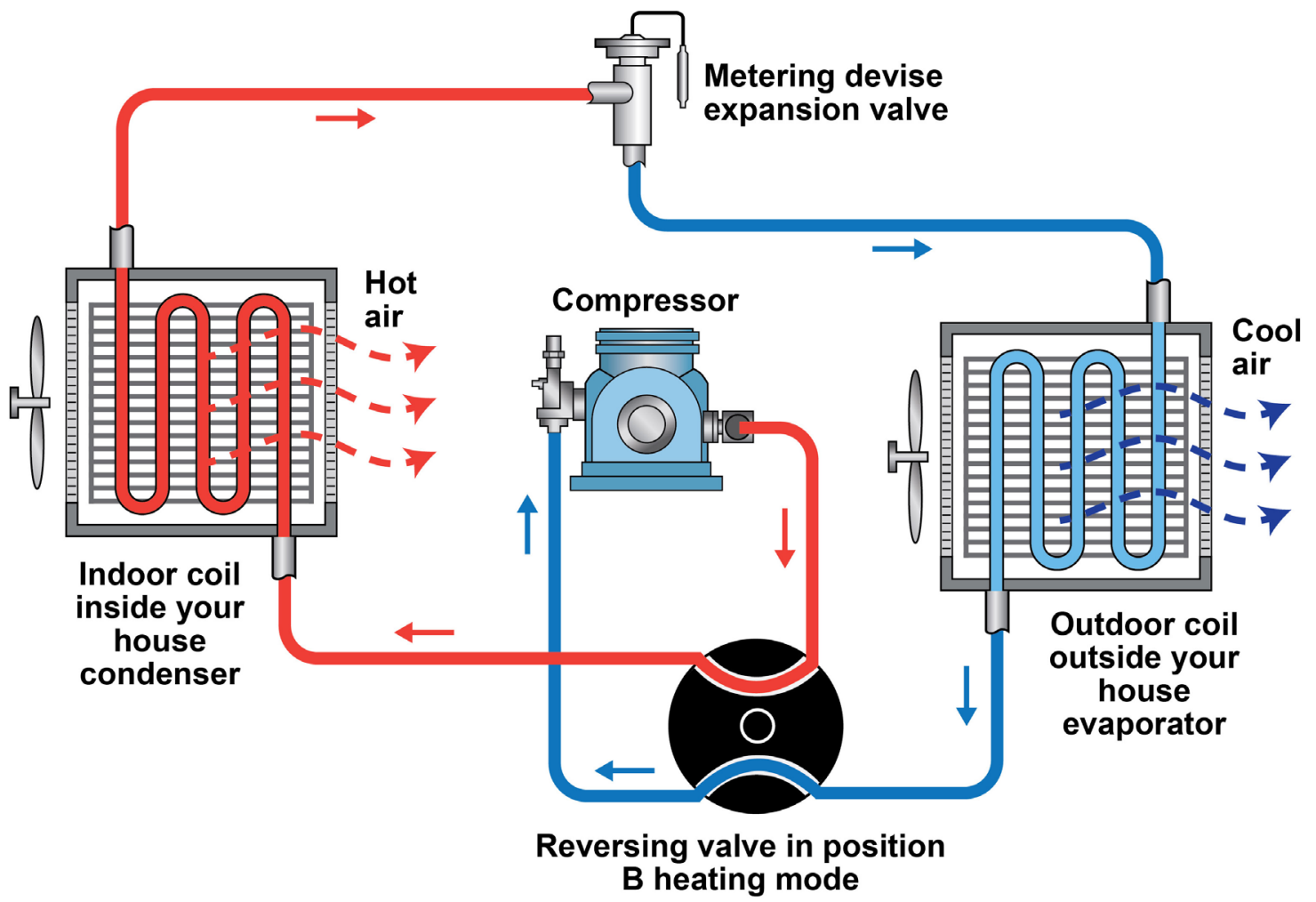

Figure 6.13. Diagram of the heat pump vapor compression refrigeration cycle. 
air in the air distribution unit, a boiler heats a secondary transfer fluid (typically water, but sometimes steam) that is in turn pumped to a series of heating coils in air distribution units, fan coils, unit ventilators, or radiation/ convection systems located throughout the building. Boilers are typically between $80 \%$ and $90 \%$ efficient in the primary process of heating the water, but any un-insulated hot water piping through unconditioned spaces may lower the overall efficiency of space heating.

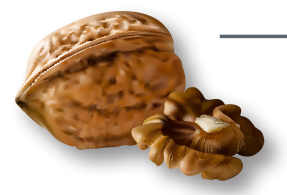

In a nutshell. . The variety

of technologies commonly available for heating use a variety of fuel types. Electric radiators and electric furnaces are typically more efficient but more expensive to operate. Heat pumps also use electricity and are more efficient, but have limitations in cold climates. Where electricity is expensive, natural gas is a good alternative for heating, either via a furnace, which is integrated into the air distribution unit, or via a boiler, which uses a separate water loop to pump heated water to heating coils throughout the building. Boilers may be designed to use a variety of hydrocarbon fuels (natural gas, propane, fuel oil, etc.). 


\subsection{LIGHTING}

Several technologies are available to provide lighting to commercial spaces and many options exist for specific lamps and fixtures Because lighting is one of the top consumers of energy in commercial buildings, it often presents major opportunities for energy savings.

Lights are used to convert electrical energy to visible light. The amount of light emitted from a lamp is measured in a unit called a

lumen. If all of the electrical energy supplied to the lighting system were converted to visible light, 1 watt of power could produce almost 700 lumens. The efficiency of today's traditional lighting technologies (e.g., incandescent, fluorescent, high-intensity discharge) in converting electrical energy to visible light, however, is only between $2 \%$ and 20\%, meaning that the actual Iuminous efficacy of lighting systems is in the range of 12 to 120 lumens per watt. Because only a fraction of the electrical energy is converted to light, most of it is converted directly to heat energy; thus, lights are a significant source of internal heat gain in buildings.

The brightness level at a work space or other target area is referred to as the level of

illumination and is measured in units of lux or foot-candles (fc). One foot candle is equal to 10.75 lux. The level of illumination is affected by the output of the lamp, the distance of the lamp from the illuminated surface, and the reflection and absorption of light from the fixture and at various surfaces in the room and from other external light sources, such as outdoor light coming in through

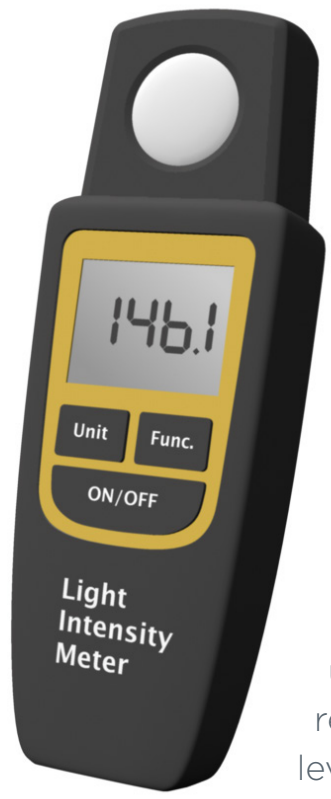
Figure 7.1. Light meter. $\quad$ minimum occupied illumination levels, but the Illuminating Engineering Society of North America (IESNA) provides recommendations for illumination levels by space type within commercial buildings in its ninth edition Lighting Handbook.? The recommendations include 400 lux (37 fc) for office spaces, 300 lux (28 fc) for conference rooms, 300 lux (28 fc) for bathrooms, and 50 lux (5 fc) for stairwells and corridors.

If there are no light meters available, a good rule of thumb for office spaces using modern linear-fluorescent lighting fixtures is that 0.9 watts of installed lighting per square foot of floor space is sufficient to provide the necessary lighting. ${ }^{8}$ For example, a 100-square-foot office space requires approximately 90 watts of linear-fluorescent lighting.

\footnotetext{
7“IESNA Lighting Handbook," by Illuminating Engineering Society of North America. 9th edition. July 2000.

${ }^{8}$ This is the maximum allowable lighting power density in the 2010 ASHRAE 90.1 standard for buildings except low-rise residential buildings. Many states and jurisdictions use either ASHRAE 90.1 or the International Energy Conservation Code (IECC) for required building codes for new construction.
} 

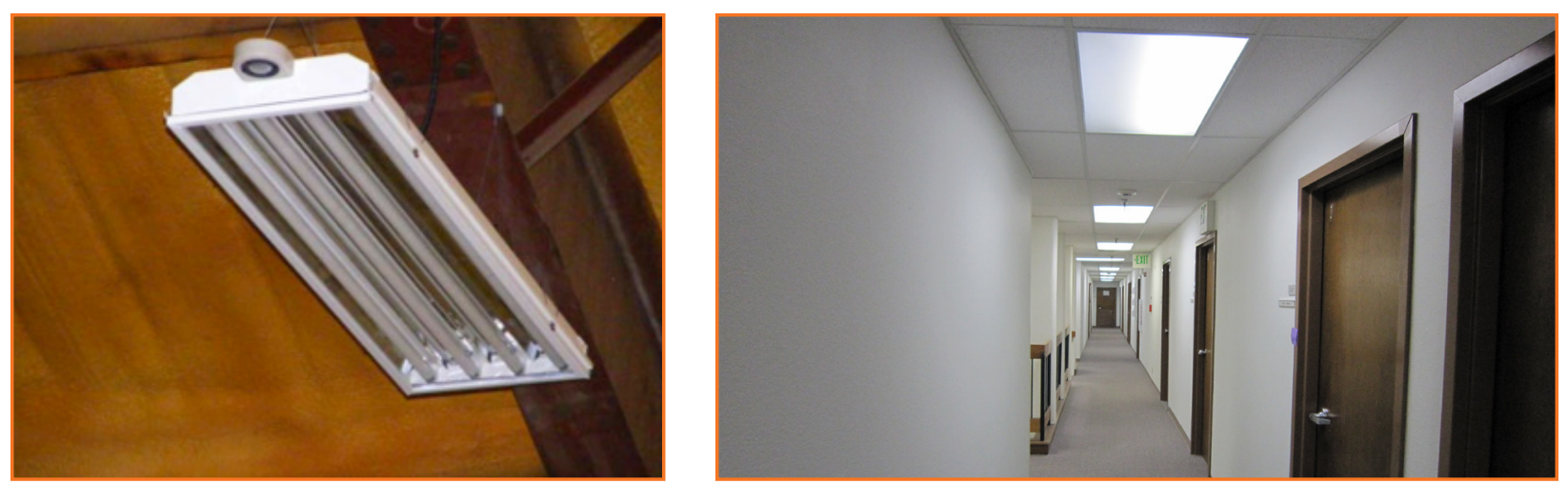

Figure 7.2. An example of a linear fluorescent fixture using only reflectors to direct light (left) and a recessed light fixture using diffusers to spread light (Image Sources [left] Courtesy of Clean Energy Resources Team; [right] PNNL).

Most lighting fixtures have four components: a lamp, socket, ballast, and reflector and/ or lighting diffuser. The lamp is the "bulb" that produces the light. The socket is the connection between the fixture and the lamp. The ballast is a device used by fluorescent and gas-discharge lamps that regulates the electric current provided to the lamp. Reflectors surround the fixture and help to aim or focus the light (reflecting the light out of the fixture and toward the space that is being lit), while diffusers spread out the emitted light so that it more effectively and evenly covers the area it is intended to illuminate. Figure 7.2 below shows a fixture (on the left) that uses only a reflector to direct the light, and fixtures (on the right) that use diffusers or diffusing lenses to spread the light evenly throughout the hallway.

The advantages and disadvantages of some of the more common types of lighting, relative to their characteristics, cost, and lifespans described in the following sections.

\subsection{Incandescent Lighting}

Incandescent lighting

uses a coiled metal resistor called a

filament within a glass bulb filled with an inert gas (see Figure 7.3). Electricity passes through the filament, causing it to heat up to high temperatures, which results in visible light being emitted. Incandescent technology dates back to the 1800 s, is the oldest form of electric lighting, and is also the

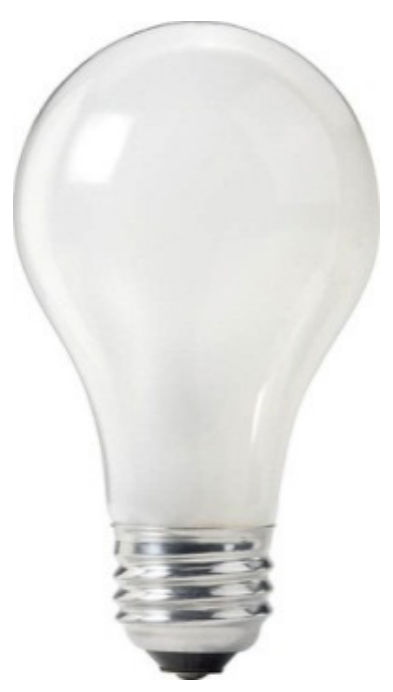

Figure 7.3. Incandescent lamp (bulb). least efficient and the most expensive in terms of energy costs. Incandescent bulbs also have very short life spans-on the order of 750 to 1,000 hours-which means a typical bulb that is on 12 hours per day, 5 days per week, will have to be replaced 35 times over a 10year period. While the bulbs themselves are inexpensive on a first-cost basis, about 94\% of the ongoing cost of using incandescent light bulbs is the electricity. The average 
annual cost for bulbs and electricity is about six times that of competing technologies. For this reason, incandescent bulbs should be replaced with compact fluorescent lamps and may only be considered acceptable in areas that are used very infrequently, like storage closets. Even in those circumstances, compact fluorescent lamps provide a good low-cost alternative.

\subsection{Fluorescent Lighting}

Fluorescent lighting produces light when an electric filament excites mercury vapor within the lamp that in turn emits ultraviolet light. The ultraviolet light causes a secondary phosphorescent vapor to emit visible light. Fluorescent lighting has been on the market since the 1930s and the technology has been refined and improved greatly over the years.

\subsubsection{Linear Fluorescents}

The most common types of fluorescent lamps in commercial buildings are linear fluorescent lamps. They come in three common sizes: $\mathbf{T}-\mathbf{1 2}, \mathbf{T}-\mathbf{8}$, and $\mathbf{T - 5}$ (see Figure 7.4). The number denotes the diameter of the bulb in eighths of an inch. T-12 lamps and fixtures were very common in earlier construction until the 1980s. Many older commercial buildings still use T-12s along with an older magnetic ballast system. T-8 lamps, however, operate with no reduction in light output, but significant energy savings. Even more energy savings come from the replacement of the magnetic ballasts with higher efficiency electronic ballasts. This is an easy retrofit because T-8s and their electronic ballasts fit into the same fixture as the old T-12 and their magnetic ballasts. A typical T-12 to T-8 retrofit for a two-lamp fixture would reduce power consumption from 74 to 48 watts (a 35\% reduction). Higher efficiency $\mathrm{T}-8 \mathrm{~s}$ are also available and reduce power consumption by as much as $42 \%$, relative to the old T-12 system.
The typical annual lamp and energy costs to light 50 square feet of office space is about $50 \%$ higher for T-12s than for T-8s. Many building owners may not know whether they have magnetic or electronic ballasts. Even when you are able to look inside the light fixture and examine the ballast it is often difficult to distinguish a magnetic ballast from an electronic ballast based on its appearances (see Figure 7.5). You can tell if you have T-12 fluorescent lighting by looking at the size of the bulbs and their bases. While both T-12 and T-8 lighting comes in standard lengths (usually about 4 feet), the diameter varies in size (1.5 inches [T-12] vs. 1 inch [T8]). Handheld tools are also now available to remotely detect magnetic ballasts. Federal laws now require the phase-out of T-12 lamps (starting July 2012) and magnetic ballasts (starting July 2010).

T-5 lighting provides an additional level of energy savings for retrofits. T-5 lamps are between $3 \%$ and $10 \%$ more efficient than T-8 lamps, but T-5 lamps do not fit directly into $\mathrm{T}-12 / \mathrm{T}-8$ sockets. However, some companies sell adaptors that eliminate the need to replace the entire fixture. Still, the lamps are currently more expensive than T-8 lamps, making the average annual combined lamp and energy cost about equal to that of T-8s.

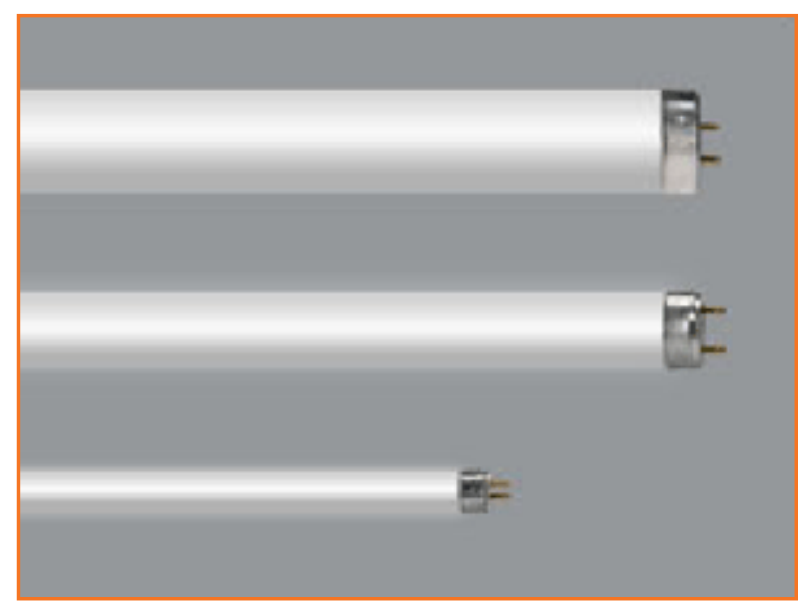

Figure 7.4. Linear fluorescent lamps in T-12 (top), T-8 (middle), and T-5 (bottom) models. 


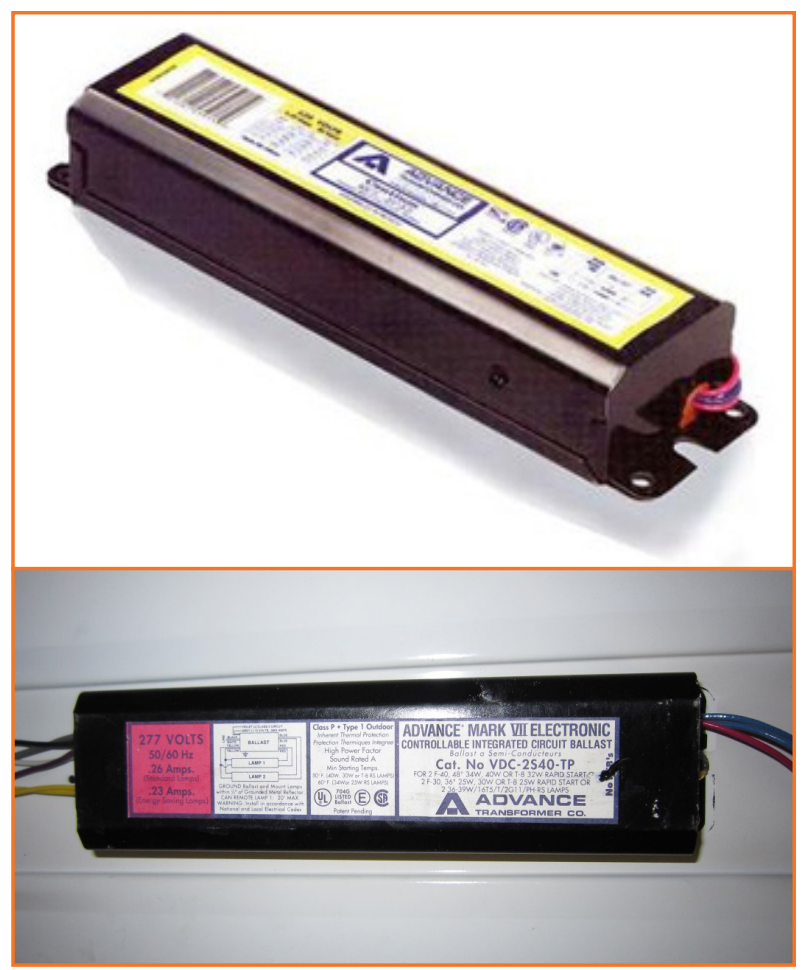

Figure 7.5. A typical electronic ballast (top) and magnetic ballast (bottom) used with linear fluorescent lights.

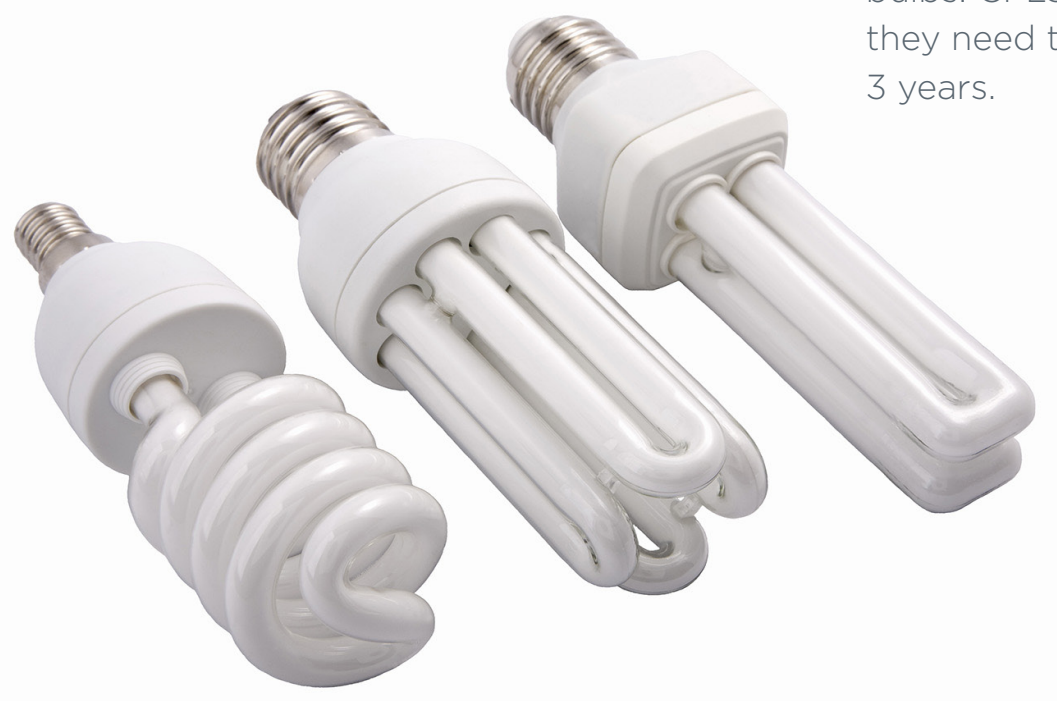

All linear fluorescent lamps last about 15,000 hours, meaning they would typically only need to be replaced every 4 to 5 years. The mercury gas contained inside the bulbs can be a health hazard if they are broken, in which case a specific cleanup protocol should be followed. At the end of their life, fluorescent bulbs should be treated with care and properly recycled.

\subsubsection{Compact Fluorescent Lamps}

Compact fluorescent lamps (CFLs) use fluorescent tubes that are similar to the linear fluorescent lamps, but they have a more compact shape (usually spiral or coiling) that seeks to replicate an incandescent bulb size (see Figure 7.6). These bulbs were developed as drop-in replacements for standard incandescent fixtures. Unlike linear fluorescents, the CFL has its ballast embedded and attached to the lamp, so no separate ballast installation is necessary. CFLs have a luminous efficacy rating of around 60 lumens per watt, making them roughly as efficient as T-12 linear fluorescent lamps. Thus, they are not as efficient as modern linear fluorescent lamps, but they still produce comparable light levels using one-quarter of the energy of incandescent bulbs. CFLs last about 10,000 hours, meaning they need to be replaced about once every 3 years.

Figure 7.6. Examples of compact fluorescent lamps. 


\subsection{High-Intensity Discharge}

Like fluorescent lighting, high-intensity

discharge (HID) lighting is another variation

of gas-discharge lighting technology.

There are two common types of HID

lighting: high-pressure sodium (HPS) and metal halide $(\mathrm{MH})$. HPS lighting has light and color rendering properties that make it unacceptable for indoor lighting, but $\mathrm{MH}$ lighting has superior color rendering properties. All HID lamps require high electric power input, but they produce very intense light compared to other lamps. The overall luminous efficacy for MH lamps can be as high as 115 lumens per watt, making them more efficient than fluorescent lamps. Using $\mathrm{MH}$ lights in a single-story application, however, would produce excessively bright illumination directly below them. One effective way to use $\mathrm{MH}$ lighting in commercial buildings is in areas with twostory ceilings or higher. The longer distance between the lamp and the target area allows the light to spread out and to take advantage of the high efficiency of these lamps. HID lighting systems, like fluorescent lamps, require the use of ballasts, which can add to the up-front and replacement costs. Average annual energy and lamp replacement costs for HID types of lighting are among the lowest of all lighting technologies-about $10 \%$ lower than T-5 or T-8 lamp costs. Lamps typically have a life span of between 20,000 and 24,000 hours, meaning they need to be replaced about once every 7 years. HID lamps are well suited for parking lots (see Figure 7.7) and street lighting.

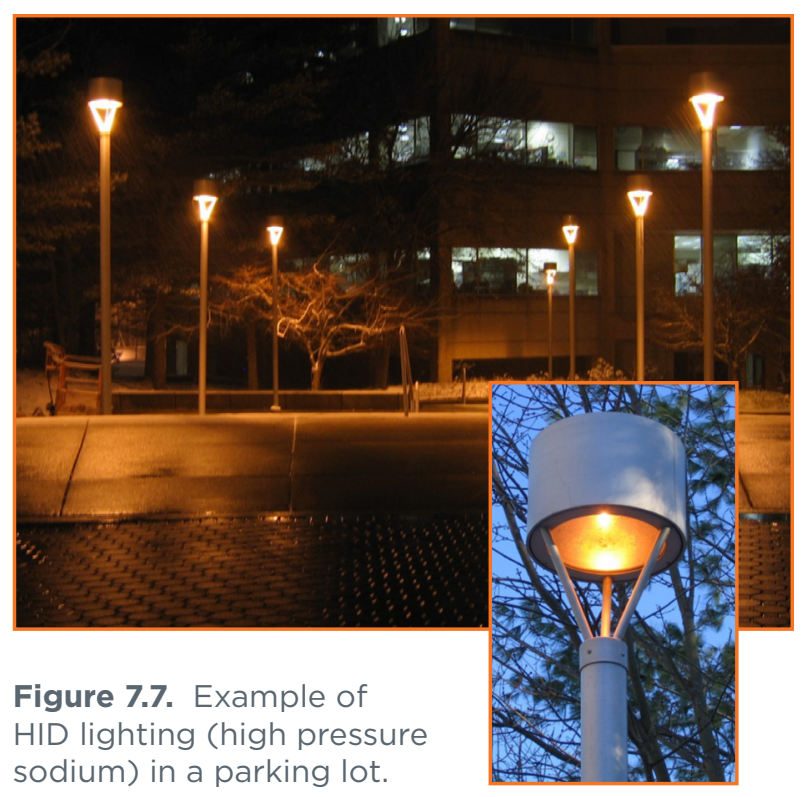

\subsubsection{Light Emitting Diode}

Light emitting diodes (LEDs) work by

passing a current through a semiconductor material that causes the emission of photons (light) at specific wavelengths and in specific directions. The best current LED lamps and luminaires have higher efficacy than fluorescent and high intensity discharge (HID) sources. LED products can have very long expected life spans, with most designed to last 25,000 to 50,000 hours at acceptable brightness levels. Currently, the biggest disadvantage of LEDs is cost. LED lamps are more expensive than other technologies. However, costs continue to decrease rapidly and LED luminaires can now compete on a life cycle cost basis in an increasing number of lighting applications, including outdoor street and area lighting, high bay lighting, recessed downlighting, and even office ambient lighting. Example applications of LED lighting are shown in Figure 7.8. 

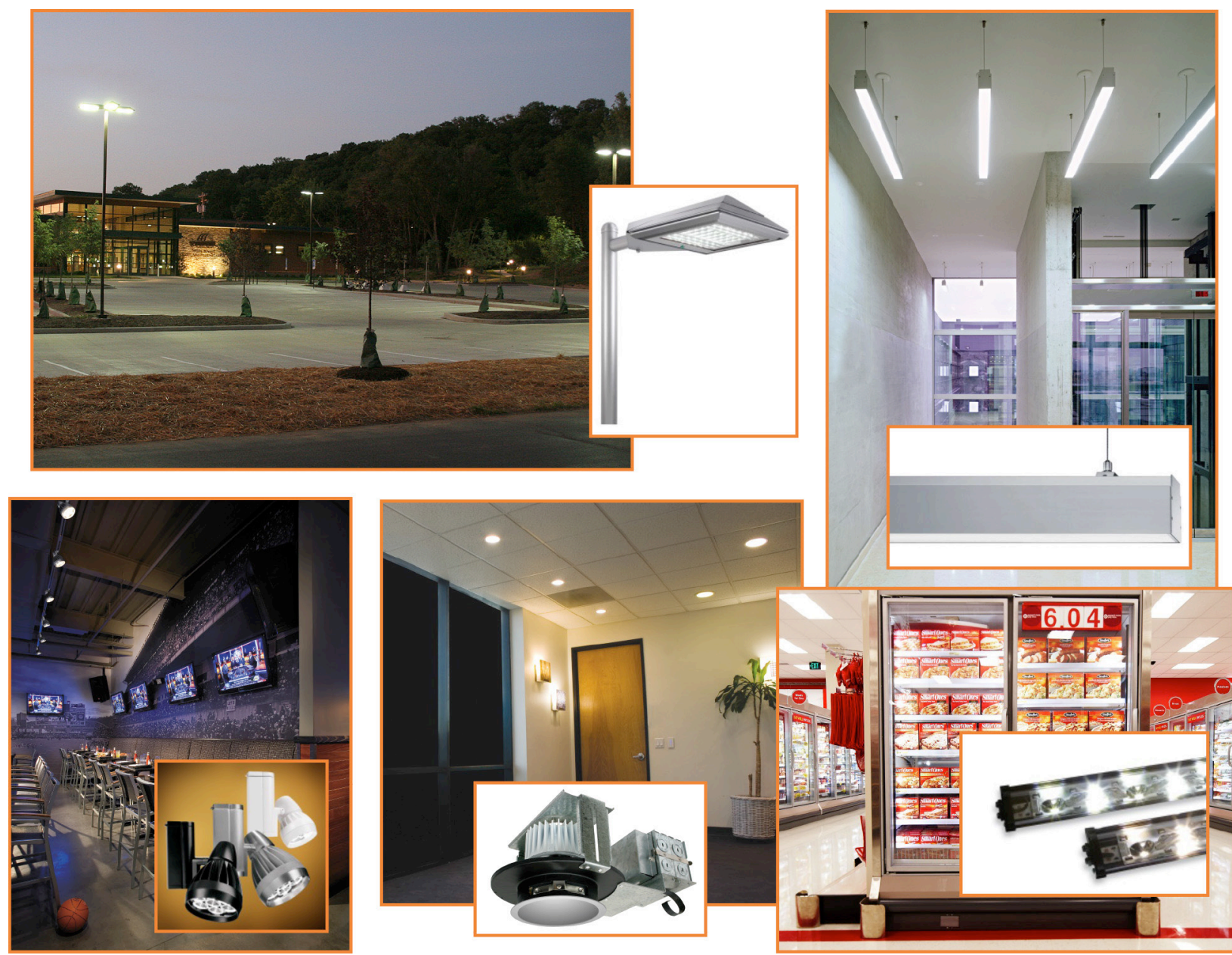

Figure 7.8. Examples and applications of LED lighting in commercial buildings (Photo Source: Next Generation Luminaires - 2009 Solid State Lighting Design Competition Winners, www.ngldc.org). 
The efficiency, life span, cost, and niche applications of the various lighting technologies are listed in Table 7.1.

Table 7.1. Characteristics of Typical Commercial Lighting Technologies'

\begin{tabular}{|c|c|c|c|c|c|c|c|}
\hline & & $\begin{array}{l}\text { High Intensity } \\
\text { Discharge }\end{array}$ & \multicolumn{4}{|c|}{ Fluorescent } & \multirow[b]{2}{*}{ LEDs $^{2}$} \\
\hline & Incandescent & Metal Halide & CFL & $\mathrm{T}-5$ & $\mathrm{~T}-8$ & $\mathrm{~T}-12$ & \\
\hline $\begin{array}{l}\text { Luminous } \\
\text { Efficacy } \\
\text { (lumens/watt) }\end{array}$ & 15 & $85-115$ & 60 & 100 & $90-97$ & $56-60$ & $70-150$ \\
\hline $\begin{array}{l}\text { Life span } \\
\text { (hours) }\end{array}$ & $750-1000$ & $22,000-24,000$ & 10,000 & 15,000 & 15,000 & 15,000 & $25,000-75,000$ \\
\hline $\begin{array}{l}\text { Lamp Cost } \\
\text { ( } \$ / 5000 \\
\text { lumens) }\end{array}$ & $\$ 2.0$ & $\$ 3.62$ & $\$ 11.57$ & $\$ 8.27$ & $\$ 3.11$ & $\$ 4.84$ & $\$ 59$ \\
\hline $\begin{array}{l}\text { Estimated } \\
\text { Annual Lamp } \\
\text { plus Energy } \\
\text { Cost ( } \$ / 5000 \\
\text { lumens-year) }\end{array}$ & $\$ 111$ & $\$ 16$ & $\$ 29$ & $\$ 17$ & $\$ 18$ & $\$ 28$ & $\$ 19$ \\
\hline $\begin{array}{l}\text { When and } \\
\text { Where to use }\end{array}$ & $\begin{array}{l}\text { Very infrequent } \\
\text { use only }\end{array}$ & $\begin{array}{l}\text { Areas with } \\
\text { high ceilings, } \\
\text { displays }\end{array}$ & $\begin{array}{l}\text { Drop-in } \\
\text { replacement } \\
\text { for } \\
\text { incandescent }\end{array}$ & $\begin{array}{l}\text { Genera } \\
\text { lighting }\end{array}$ & ffice & None & $\begin{array}{l}\text { Focused/ } \\
\text { task lighting } \\
\text { when energy } \\
\text { performance is } \\
\text { main priority }\end{array}$ \\
\hline
\end{tabular}

In a nutshell. .. A variety of lighting technologies are available to meet a building's illumination requirements, and some technologies are better-suited to the requirements than others. In addition to producing visible light, lighting produces waste heat, which contributes to the internal heat gain of the building. Energyefficient lighting alternatives reduce the amount of energy needed to produce light, may reduce the amount of waste heat produced, and tend to last longer, making them more cost effective on a life-cycle basis.

'These estimates were developed by PNNL based on the middle of the given range for luminous efficacy and typical operating lifetime. The actual life of each lighting technology assumes 60 hours per week of use. The cost calculated is the average annual cost of initial lamp cost plus replacement lamps over a 10-year period, plus the annual cost of electricity at 60 hours of use per week and at 10 cents per kWh. Lamps costs are based on current (2013) costs for standard sizes in bulk purchases (20-60 lamps) from major retailers.

${ }^{2}$ Note that LEDs represent a rapidly evolving technology in terms of luminous efficacy and cost. Costs have been calculated based on the A19, 12-watt lamp. For more information on LEDs, visit DOE's Solid-State Lighting website: 
Small Commercial Building Re-tuning: A Primer

0
$z$
$E$
$\frac{1}{0}$
1
0
$N$ 


\subsection{HOT WATER SYSTEM}

In commercial buildings where large amounts of hot water are used, the energy used for heating water can be a significant component of a building's total energy consumption. For example, in the lodging industry, 31\% of energy is used for water heating. ${ }^{9}$ Other commercial buildings with heavy hot water demand include restaurants, commercial laundries, buildings with industrial processes, and buildings such as dormitories or other high-density housing facilities.

Within the United States, most water heating is accomplished through the use of a conventional storage water heater. Other types of water heaters include heat pump water heaters (which typically also use storage tanks), on-demand water-heating systems (also referred to as tankless or

instantaneous water heat), and solar waterheating systems.

\subsection{Storage Water Heaters}

Most commercial water heating is done with tank storage water heaters that use gas, oil, or electricity to heat the water. Other less common energy sources can include steam, solar, wood, waste heat, etc. Figure 8.1 shows a picture of a typical upright storage waterheating system. Conventional storage water heaters use a tank to heat and store hot water so that it is continuously ready to use. A storage water heater operates by releasing hot water from the top of the tank when the hot water tap is turned on. To replace that hot water, cold water enters the bottom of the tank, ensuring that the tank is always full. The sizes of storage water heaters range from 20 gallons to hundreds of gallons. Storage water heaters remain the most popular type for water heating in the United States.

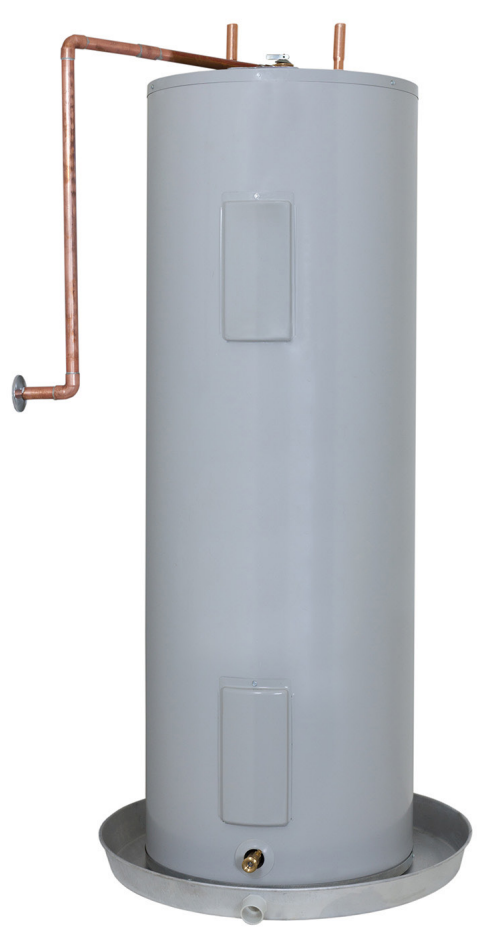

Figure 8.1. Common storage water heater tank.

Because the water is constantly heated in the tank, energy can be wasted even when no faucet is on. This is called standby heat loss. Storage tanks typically have a layer of insulation between the shell and the inner tank; however, depending on the age of the tank, the installation of extra insulation-a water heater blanket-can provide additional protection against standby heat loss. Newer, more energy-efficient storage models can significantly reduce the amount of standby heat loss, making them less expensive to operate.

Hot water pipe insulation may also be installed; however, care must be taken to install it properly around a gas-fueled water heater because of potential flammability 
issues. The installation or use of pipe insulation reduces heat loss from the pipes, which can increase the delivered water temperature by a few degrees, potentially allowing the user to reduce the temperature of the water heater.

\subsection{Heat Pump Water Heaters and Desuperheaters}

Heat pump water heaters (HPWHs) use the same principle of transferring heat as the heat pump described in the HVAC section, except that they extract heat from air (indoor, exhaust, or outdoor air) and deliver it to water. Some models come as a complete package, while others work as an addition to a conventional water heater. Because it extracts heat from air, the HPWH delivers about twice the heat for the same electricity cost as a conventional electric resistance water heater. A variation of the standalone HPWH is the desuperheater feature available on some central air conditioners. Desuperheater water heating can be part of an integrated package with a heat pump or air-conditioning system. In most such systems, the heat pump water heating only occurs during normal demand for space conditioning, and resistance electric coils provide water heating the rest of the time. During the cooling season, the desuperheater actually improves the efficiency of the airconditioning system while heating water at no direct cost.

\subsection{Tankless Water Heaters}

On demand, or tankless, water heaters are more commonly seen in Japan and Europe, but have been increasing in popularity in recent years in the United States. Unlike conventional tank water heaters, tankless

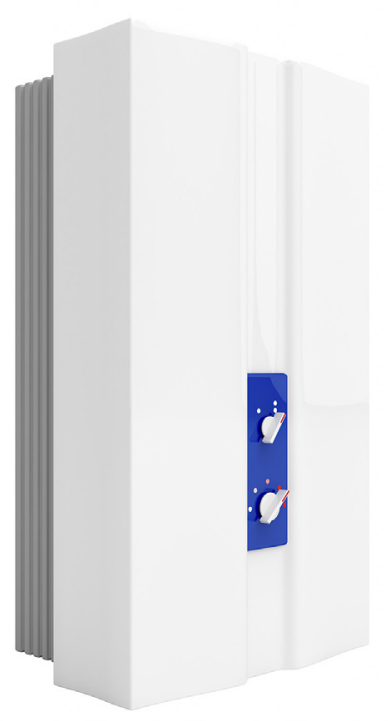

Figure 8.2. On-demand water heater.

water heaters heat water only as it is used, or on demand. A tankless unit has a heating device that is activated by the flow of water when a hot water valve is opened. Once activated, the heater delivers a constant supply of hot water. The output of the heater, however, limits the rate of the heated water flow. Figure 8.2 illustrates a typical ondemand heating unit.

On-demand water heaters are available in propane (LP), natural gas, or electric models. They come in a variety of sizes for different applications, such as a whole-building water heater, a hot water source for a remote bathroom or hot tub, or as a boiler to provide hot water for a heating system. They can also be used as a booster for dishwashers, washing machines, and solar or wood-fired hot-water systems. These systems also may result in a lower demand for hot water because users don't need to leave the faucet running while they wait for hot water to be delivered. 


\subsection{Solar Water Heaters}

An estimated 200,000 commercial solar water-heating systems have been installed in the United States. ${ }^{10}$ Although there are a large number of different types of solar water-heating systems, the basic technology is very simple. Sunlight strikes and heats an "absorber" surface within a "solar collector" or an actual storage tank (see Figure 8.3). Either a heat-transfer fluid or the actual potable water to be used flows through tubes attached to the absorber and absorbs the heat. Systems with a separate heattransfer-fluid loop include a heat exchanger that heats the potable water. The heated water is stored in a separate preheating tank or a conventional water heater tank until it is needed. Solar water heater systems may also have freeze or overheating protection capabilities installed if there is a possibility of the potable water or collector fluid freezing or overheating. Freeze protection can be provided through the use of freeze-tolerant collectors that expand upon freezing, a drain-back system, the manual draining of collectors when freezing is expected, or use of antifreeze in the heat transfer fluid. During

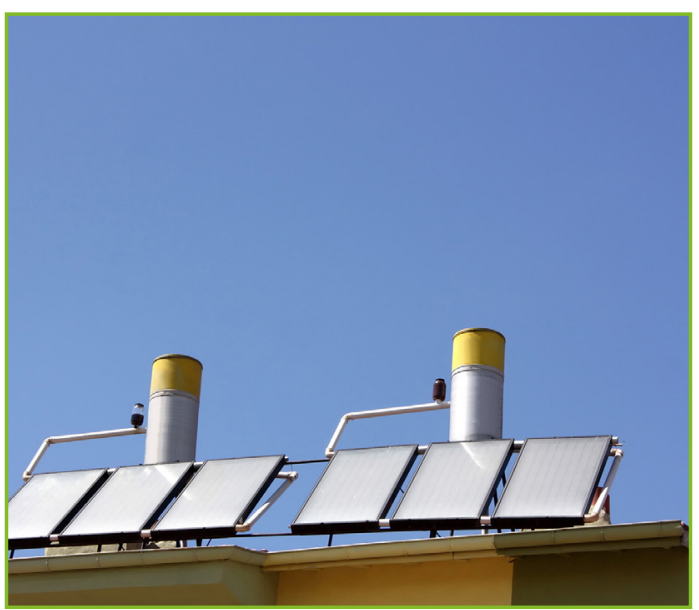

Figure 8.3. Solar panels and storage tank associated with a solar water-heating system.

10 U.S. Department of Energy - Energy Efficiency and Renewable Energy, Building Technologies Program - Commercial Buildings: Water Heating http://www1.eere.energy.gov/buildings/commercial/printab/e_versions/water_heating.html

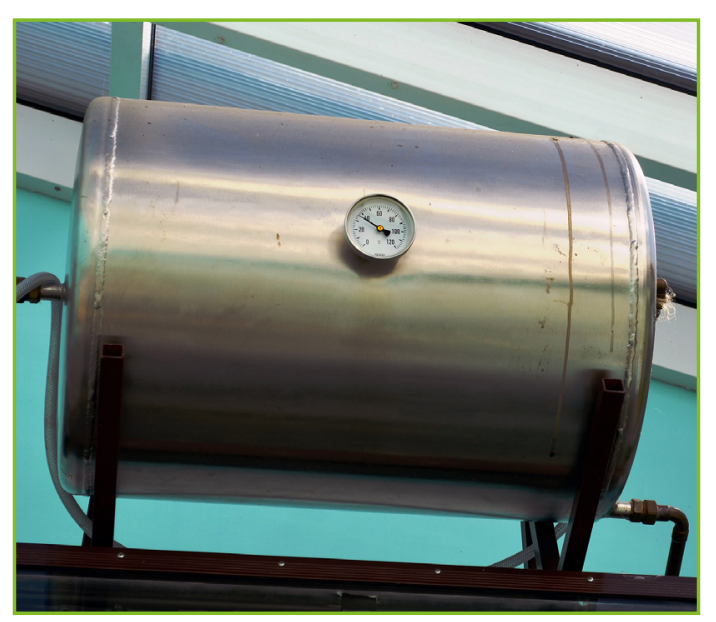

periods of low hot water load, overheating can become a problem depending on the type of system; therefore, overheating protection is usually provided through a drain-back system, use of a temperature and pressure relief valve, or circulation of cooler water during non-heating periods (such as nighttime or little sunlight). If additional heat is needed, it is provided by a conventional water-heating system fueled with electricity or fossil fuels. By reducing the amount of heat that must be provided by conventional water heating, solar water-heating systems directly substitute renewable energy for conventional energy, reducing the use of electricity or fossil fuels by as much as $80 \%$.

\subsection{Storage Water Heater Operation}

Storage water heaters have a number of parts that help monitor and maintain the operation of a water heater. These include temperature controls, shutoff valves, temperature and pressure relief valves, drain valves, sacrificial anodes, and dip tubes. Figure 8.4 shows the typical parts of a storage water heater. 


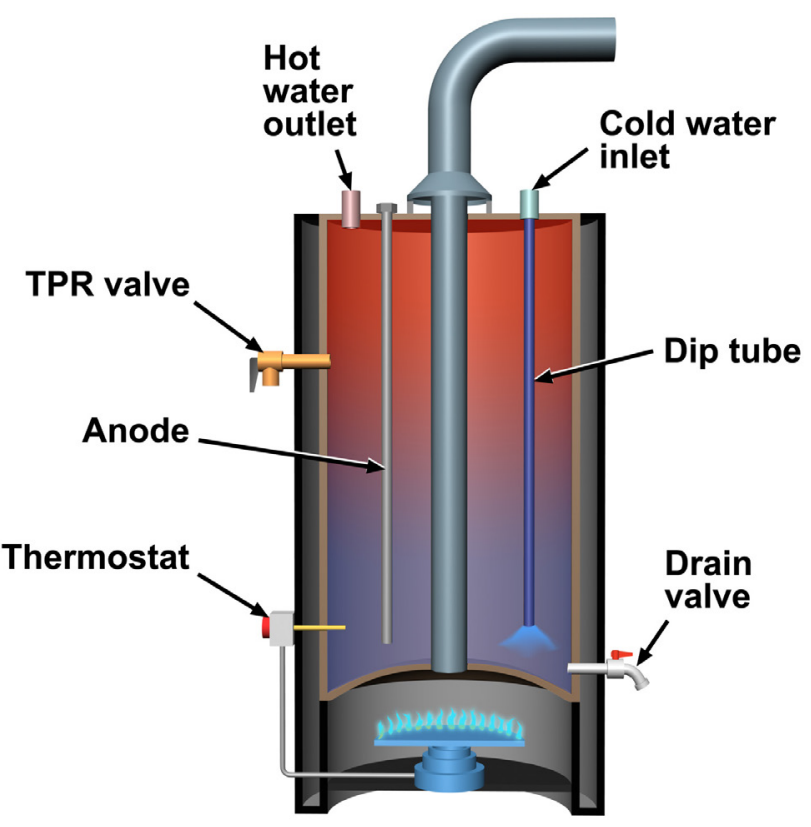

Figure 8.4. Diagram of a gas water heater.

Generally, water heaters have a thermostat that monitors the temperature of the water near the bottom of the tank and turns on a burner or heating element to heat the water to the desired temperature (electric water heaters often have a second thermostat in the upper part of the tank). When the thermostat senses that the water temperature has fallen below a set temperature, the thermostat triggers the burner or heating element to come on. For electric water heaters, the upper thermostat activates the upper element first and when

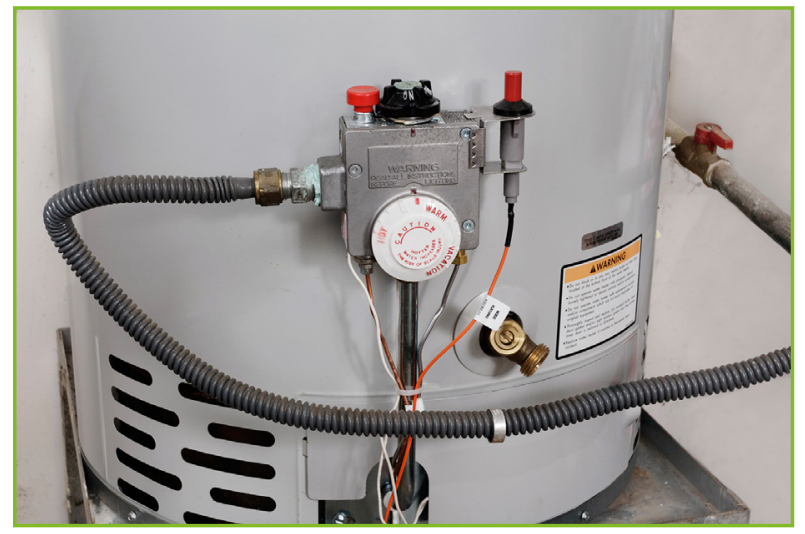

it senses that the water has been heated to the set temperature, the lower thermostat is allowed to activate the lower element until it reaches the set temperature. Electric water heaters also have a high-limit switch located in the upper thermostat, which acts like a circuit breaker. If the water temperature gets too hot, the high-limit switch is tripped and must be manually reset. The thermostat does not control the outlet temperature (the temperature at the faucet). Water heater burner thermostat controls can have temperature swings of 20 to 30 degrees between "energy on" and "energy off," and use of the water heater burner thermostat to control outlet temperature is ineffective.

Not all water tanks have thermostats marked with degrees; some dials are labeled "hot" and "warm" or "high" and "low." Generally, the lower settings heat water to between $90^{\circ} \mathrm{F}$ and $110^{\circ} \mathrm{F}$ and the higher settings heat water to between $140^{\circ} \mathrm{F}$ and $150^{\circ} \mathrm{F}$. For tanks with more than one thermostat, the thermostats should be set at the same temperature. To test for the approximate water tank temperature, allow the water heater to sit unused for 1 hour and then turn on the hot water faucet closest to the water heater and let the hot water run for at least 1 minute. Once the hot water has been running long enough to come up to temperature, fill a cup with the hot water and use a cooking thermometer to measure the temperature. Figure 8.5 depicts some temperature controls.

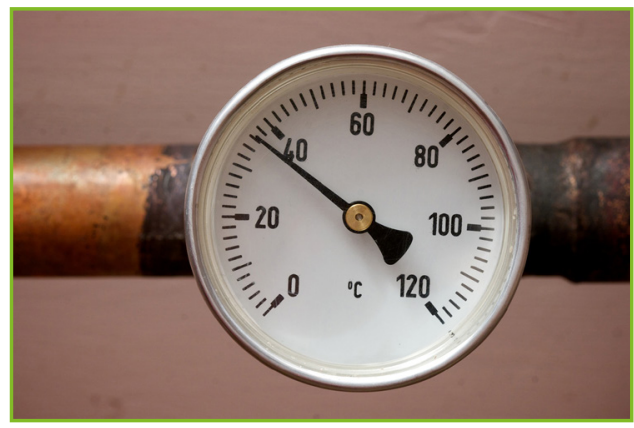

Figure 8.5. Water temperature controls and thermometer on an intake pipe. 
There is currently some debate regarding the appropriate temperature setting for a water heater. While a lower temperature $\left(120^{\circ} \mathrm{F}^{11}\right)$ is adequate for most purposes, saves energy, and prevents scalding, a higher temperature $\left(140^{\circ} \mathrm{F}^{12}\right)$ minimizes or kills waterborne bacteria, but may increase scalding or leaching of lead from lead pipes into the water. While a tank heated to $120^{\circ} \mathrm{F}$ is considered safe for most people, individuals with a suppressed immune system or chronic respiratory disease may want to consider heating to higher temperature and installing a thermostatic mixing valve (which mixes cold water with hot water to reach a set temperature) or other temperature-regulating devices to minimize scalding. Hot water temperature requirements may be regulated by local, county, or state codes.

Water is supplied to the water tank through a cold-water supply line, which is controlled by a shutoff valve. Prior to performing maintenance on the water tank, the water supply should be shut off. If the water heater is fueled by natural gas or propane, there will also be a gas line shutoff valve. If the water heater is electrically powered, there should be a circuit breaker that should be turned off. Follow all mandated safety procedures (lock and tag, etc.) prior to working on any hotwater tank.

The temperature and pressure relief valve (TPR, T/P, or T\&P valve) is a safety feature usually found on the top of the water tank, although it is sometimes mounted on the side of the tank. If the water temperature or pressure inside the hot-water tank increase and approach the safety limits of the tank's maximum design range, this valve opens to relieve the extra temperature or pressure.
Depending on the mineral content and natural debris (such as sand) in the intake water, sediment can build up in the bottom of the water tank. While a small amount of sediment is not harmful, a large buildup of sediment can clog the drain valve, slow the heat transfer from the burner to the water, and cause overheating in the bottom of the tank. The tank drain valve, located near the bottom of the tank, is used to drain the hotwater tank and any sediment that has built up, which increases the life of the water tank and improves water quality.

Storage tanks also contain a sacrificial anode-a metal rod made from a highly active metal (usually magnesium or aluminum to which corrosive elements are drawn)-to help prevent corrosion of the tank itself. The sacrificial anode is screwed into the top of the tank. Storage tanks also contain a dip tube-a long, slender plastic tube with a small hole near the top-which directs the incoming cold water to the bottom of the tank. The dip tube drops down into the inlet fitting and keeps the incoming cold water from prematurely mixing with the heated water. A break in the dip tube allows cold water to enter the hot water outlet, resulting in a lower water temperature at the faucet.

Regular inspection, maintenance, and repair of water heater system parts allow the system to perform as efficiently as it was designed to perform. Installation of additional insulation, a thermostatic mixing valve, monitoring and fixing of leaks in pipes and fixtures, purchase and installation of energy- and water-efficient appliances, and proper use and maintenance of aerators and low-flow fixtures also help to decrease the amount of hot water needed, thereby saving water-heating energy.

"See DOE's Energy Saver article on water heating for more information:

http://energy.gov/energysaver/articles/tips-water-heating

${ }^{12}$ See U.S. Department of Labor's Occupational Safety \& Health Administration Frequently Asked Questions for more information: http://www.osha.gov/dts/osta/otm/legionnaires/faq.htm/ 
Small Commercial Building Re-tuning: A Primer

All exposed and accessible piping that transports hot water (whether for domestic use or for heating use) should be insulated once the piping leaves the hot-water tank (but to mitigate fire hazards do not insulate near any flue or open flame areas).

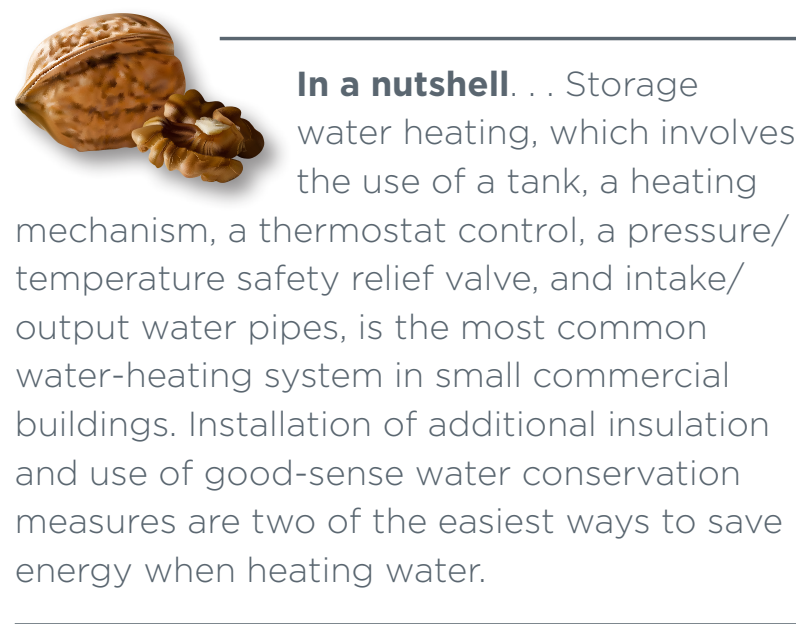




\section{(“曲) 9.0 METERS, SENSORS, AND CONTROLS}

Meters and sensors are devices that collect information about the state of a building and the systems in the building. Meters can be thought of as "counting" sensors that collect data about energy or water consumption over a period of time. Some meters may be used for internal observation of individual buildings (in the case of a campus) or even individual pieces of equipment; however, for most buildings, individual meters are used to measure the use of electricity, natural gas, and domestic water at the building level, and each meter is installed and monitored by the respective billing utility. In addition to these "counting" sensors, most buildings have a variety of other sensors that provide a "snapshot" of building system performance. They are used as either feedback devices by the HVAC or lighting system to automatically control the building, or as monitoring devices to alert the building operator to unusual or unsafe conditions. Typical building "snapshot" sensors may measure the current temperature, humidity, air flow rate, carbon monoxide, light levels (photocell), and movement (occupancy sensor) at different places in the building or in the HVAC system.

\subsection{Meters}

Most people are familiar with the concept of meters from living in a metered house or apartment. Meters are usually found on the exterior of the building so that the utility can easily access them for observation (reading) or replacement. Three types of meters are commonly used in small buildings: electric, natural gas, and water meters.

\subsubsection{Electric Meters}

Electric meters measure cumulative electricity consumption, usually reported over the course of 1 month. The unit for electricity consumption used for virtually all electricity meters and utilities is kilowatthours (kWh), which is the integration (or sum over time) of instantaneous power consumption (in kilowatts). For example, if a building uses electricity at a rate of 100 $\mathrm{kW}$ for 10 hours of the day and at a rate of $25 \mathrm{~kW}$ for the other 14 hours of the day, the electricity consumption is $100 \mathrm{~kW}^{*} 10$ hours $+25 \mathrm{~kW}^{*} 14$ hours $=1,350 \mathrm{kWh}$. Many electric utilities bill energy use based on both the total monthly energy consumption (kWh) and the maximum power consumption ( $\mathrm{kW}$ ) recorded over the billing period, or (alternatively) during the entire previous year. Most electric utilities are slowly switching to interval meters (commonly referred to as smart meters), which provide the same consumption information at a higher resolution (i.e., more frequent time intervals). In addition to utilities using electric meters for billing purposes, electric meters are used for individual equipment. Larger commercial buildings, for example, may have HVAC units and systems with built-in electric meters that assist in monitoring and controlling pumps, fans, and chillers. For smaller buildings that have packaged HVAC units and do not have automated central control of HVAC systems, such electric meters generally are unavailable (unless they are installed as separate items; for instance, as part of an updated control system scenario). For building managers who are interested in measuring power consumption from smaller plug-in devices, however, inexpensive consumer devices, like the "Kill-A-Watt" meter shown in Figure 9.1, can serve as a plug-in metered interface between a standard 120-V outlet and an electricity-consuming device to track power and energy consumption. 


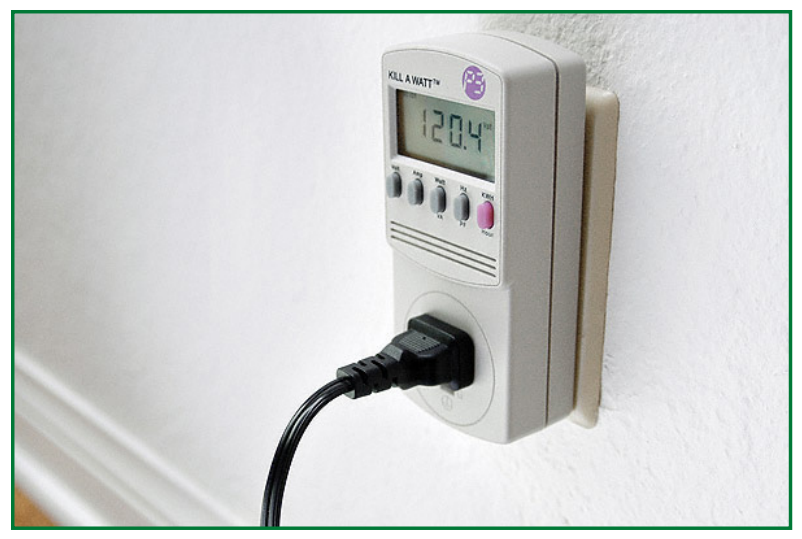

Figure 9.1. Kill-A-Watt power meter (Source: Courtesy of P3 International).

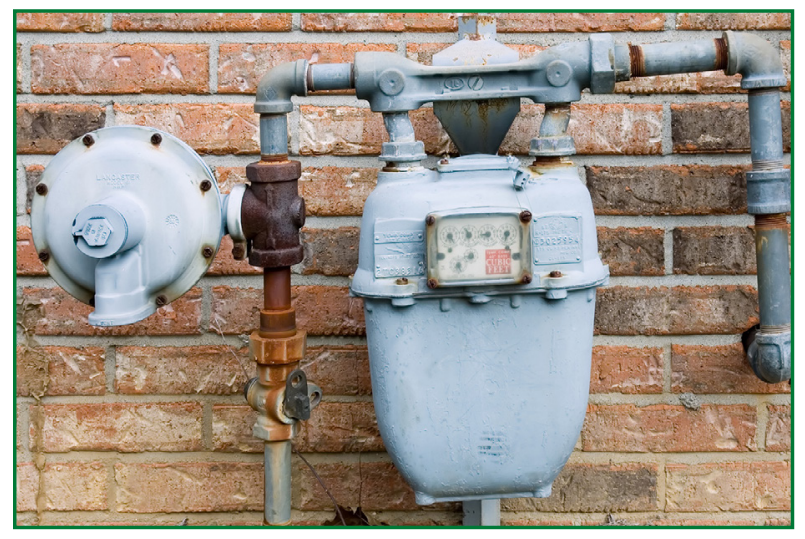

Figure 9.2. Natural gas meter.

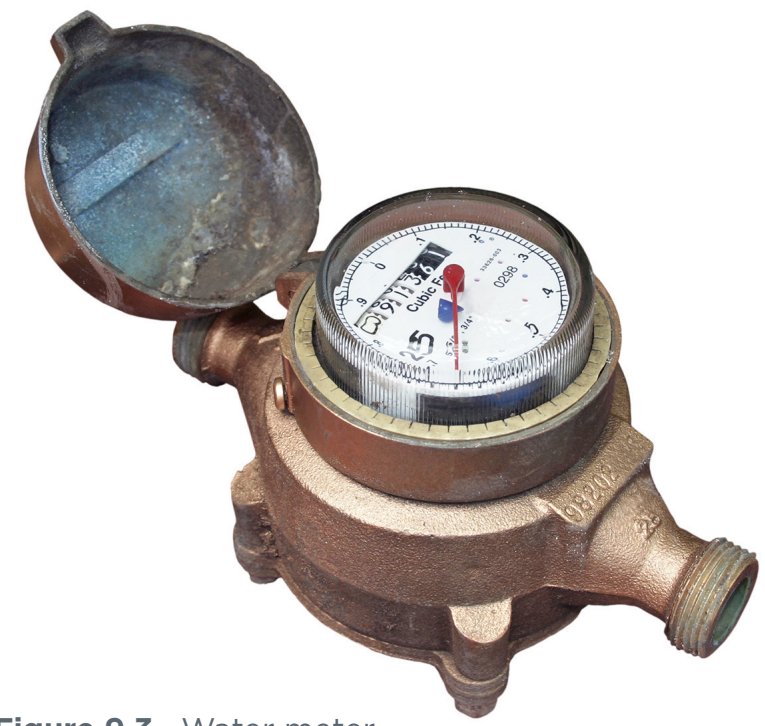

Figure 9.3. Water meter.

\subsubsection{Natural Gas Meters}

Natural gas meters, like the one pictured in Figure 9.2, typically only exist as utility metering devices at the building or campus level. They exist at the interface between the building and the utility's municipal distribution pipeline. Many buildings do not have a natural gas connection or service and use only electricity for energy consumption. Natural gas meters use either therms or cubic feet (CF) as the unit of consumption. Therms are a measure of the energy content in the fuel. For example, 1 therm is equal to the consumption of $100 \mathrm{CF}$ of natural gas and approximately equal in energy content to $29.3 \mathrm{kWh}$

\subsubsection{Water Meters}

Like natural gas meters, water meters, like the one pictured in Figure 9.3, usually only exist as utility metering devices at the building or campus level. A utility may use a single water meter to charge for both domestic water supply and sewer services. A common unit of measurement for water meters is gallons, and specific meters may read in increments of 10 , 100 , or 1000 gallons. In many installations, the water meter is installed at or near ground level.

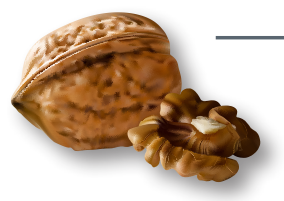

In a nutshell. . . Meters

are sensors that count consumption. For most

buildings, the only meters are those installed and operated by utilities for tracking energy and water consumption on a monthly basis. Meters may also be installed at the building level or on specific pieces of equipment so building operators can observe consumption patterns within the building. 


\subsection{Sensors and Control Systems}

While meters, as a type of sensor, are most commonly used to count cumulative consumption at the whole-building level, other types of sensors are used for internal control and monitoring. One of the first steps in troubleshooting problems with building control systems is to understand their sensors and the implications of a sensor failing. Two common "pathologies" of faulty sensors are erratic sensors and biased sensors. An erratic sensor has readings that excessively jump around, rather than a stable reading that reflects the underlying measurement. This can be caused by a poorly configured or loose or broken electrical connection. A biased sensor is a sensor that gradually falls out of calibration and loses its accuracy over time. For example, a new temperature sensor may read the correct temperature to within 1 degree, but over time, it may read consistently too high by 5 degrees or more. This is called a bias because the sensor will always be "off" by nearly the same amount in one direction from the true reading. Increased bias can be caused by the accumulation of dust, which acts as an insulator.

Control systems are composed of a sensor, an actuator, and a controller that takes feedback from the sensor to control the actuator. The actuator is a device that is connected to a damper or a coil that provides a physical response to deliver the desired set point condition. The set point may be a desired temperature, relative humidity, light level, etc. The logic and communication governing the interaction between the sensor and the actuator takes place at a controller. Often, in small buildings, the controller is embedded in a piece of equipment like a thermostat serving a rooftop unit or an economizer controller serving the same rooftop unit.
This section focuses on several types of sensors and control systems found in commercial buildings.

\subsubsection{Temperature Sensors and Temperature Control Systems}

Temperature sensors are found throughout commercial buildings and provide important feedback about temperatures that guide a variety of HVAC control systems.

Temperature sensors usually consist of a wired junction between two dissimilar metals. As the temperature changes, the electric resistance at the junction changes in a predictable way. Because they are relatively simple devices with no moving parts, temperature sensors tend to be common and inexpensive. Temperature sensors are typically found in the following places in commercial buildings:

- In zones - Zone sensors measure the temperature of rooms and interior spaces and are connected to a thermostat.

- Outdoor air - An outdoor air sensor measure the outdoor air temperature (and humidity if enthalpy is a desired control set point response) to control an economizer. It needs to be placed in an area that is shaded so that the sun does not warm the sensor beyond the actual outdoor air temperature. The sensor should also be placed away from other heating sources, such as chimneys, exhaust vents, or dark roofs and pavement, to avoid false outdoor temperature readings.

- HVAC ductwork - Temperature and humidity sensors can be found in the return air ductwork that returns air from the zones. Temperature sensors can be found in the mixing box (mixing of the return air and the outdoor air) for economizer control, in the supply air stream, and at the discharge terminal unit that supplies conditioned air back into the zone. For large HVAC ductwork, the 
most accurate temperature sensors are averaging sensors that are designed to have their sensing element span across the area of the duct in a diagonal fashion. This can be important when duct design causes stratification of warm and cool air (for example, downstream of the mixing box, cold outdoor air may settle at the bottom of the duct and warm return air may ride on top until the two air streams are naturally mixed). The cost of averaging sensors is higher than that of standard sensors. Standard sensors are usually configured to be no more than 8 to 16 inches long and therefore only sense a small section of the HVAC duct, while averaging sensors can be as long as 17 feet.

\subsubsection{Thermostats}

A thermostat is a controller that activates the fan blower and the heating/cooling on a rooftop unit. A thermostat can be used to activate the heating valve of a reheat coil that is part of an air terminal box and/or the amount of air flow to a VAV air terminal box. The air flow amount is based on a zone temperature sensor and its deviation from the zone temperature set point. A thermostat allows you to set the temperature set point that the HVAC system will try to maintain in the zone controlled by the thermostat. By design, a dead band value is typically programmed into the thermostat's configuration parameters. The dead band value is the change in temperature above and below the target set point(s) that will keep the HVAC system from initiating heating or cooling. Without a sufficiently wide dead band value, the HVAC system can easily cycle back and forth between heating and cooling because of inherent delays in heating and cooling to affect the thermostat's temperature sensor. For example, if the single set point is $72^{\circ} \mathrm{F}$, the system might initiate cooling at $72.5^{\circ} \mathrm{F}$ and initiate heating at $71.5^{\circ} \mathrm{F}$ (a 1-degree dead band). Most rooftop units also have built-in minimum runtime timers to help mitigate equipment shortcycling (where the HVAC unit has either very short runtimes or short off times), especially in commercial applications. Maintaining the targeted dead band values is critical to preventing excessive equipment wear and tear (and saving energy). The current industry standard is a 3- to 4-degree dead band to enhance both energy efficiency and equipment life.

If a thermostat has two temperature set points, the lower set point is the heating set point and the higher set point is the cooling set point. The difference between the two set points is called the thermostat's dead band. The HVAC system activates its heating when the temperature drops below the heating set point or activates its cooling when the temperature rises above the cooling set

point. If the temperature is between the two set points (in the dead band), the HVAC system will not activate its heating or cooling.

Placement of the thermostat can be very important for effective zone temperature control. The thermostat can only sense the temperature in the location where it is placed (unless it is a newer model capable of having remote wired or wireless sensors connected to it). Often, a single thermostat controls heating or cooling to several enclosed offices or other spaces that may have different thermal needs and characteristics. If the thermostat is placed in a location that is unusually hot or cold (compared to the surrounding spaces), it can cause problems for the rest of the surrounding spaces because the thermostat may cause overcooling or overheating in those surrounding spaces. Also, if there is a source of heat next to the thermostat, like a computer, the thermostat will sense that the temperature of the room is hotter than it actually is. With the advent of wireless sensors that can provide additional 

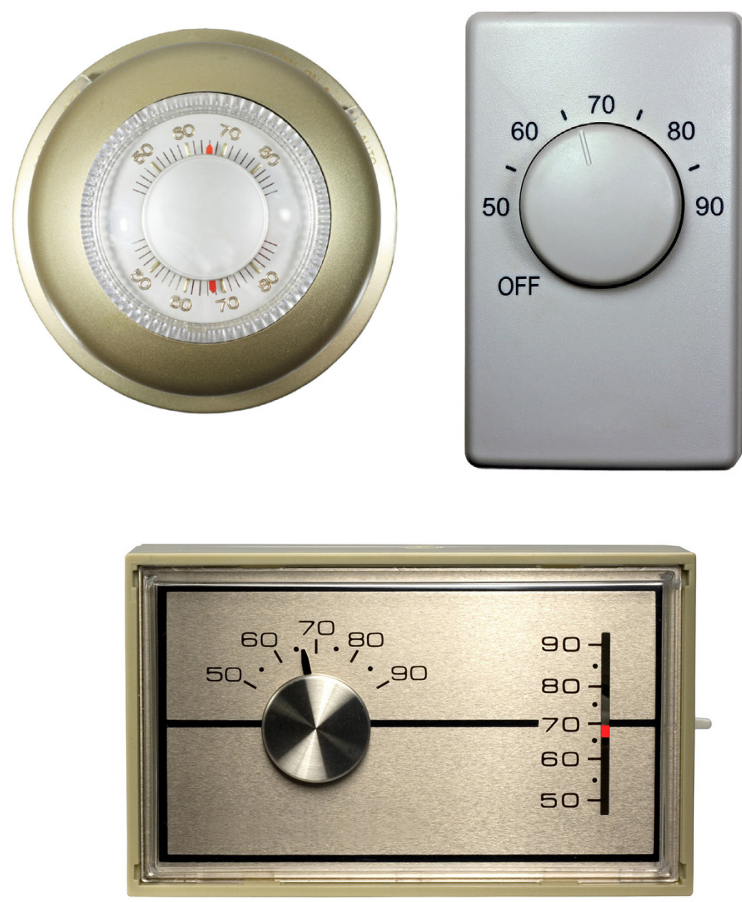

Figure 9.4. Non-programmable thermostats.

input(s) to newer thermostats, the days of fixed sensing (versus mobility) and poor temperature sensing (hot/cold problems) caused by location issues should be a thing of the past, but upfront investment is needed to move the dial in this direction.

Over the last few years, the market has been flooded with web-enabled thermostats that communicate wirelessly or via local network connection with the Internet. This enables building owners and occupants to remotely access the thermostats that maintain their interior comfort conditions. It also allows for connection (or connectivity) to other tools and remote diagnostics of the HVAC systems for things like runtime hours, efficiency degradation, maintenance recommendations, and other equipment monitoring and improvement suggestions. This moves the paradigm from "reactive maintenance" to "proactive maintenance" (discussed later). Perhaps of greater value is the ability to remotely change the occupancy schedule or the heating and cooling set points (as

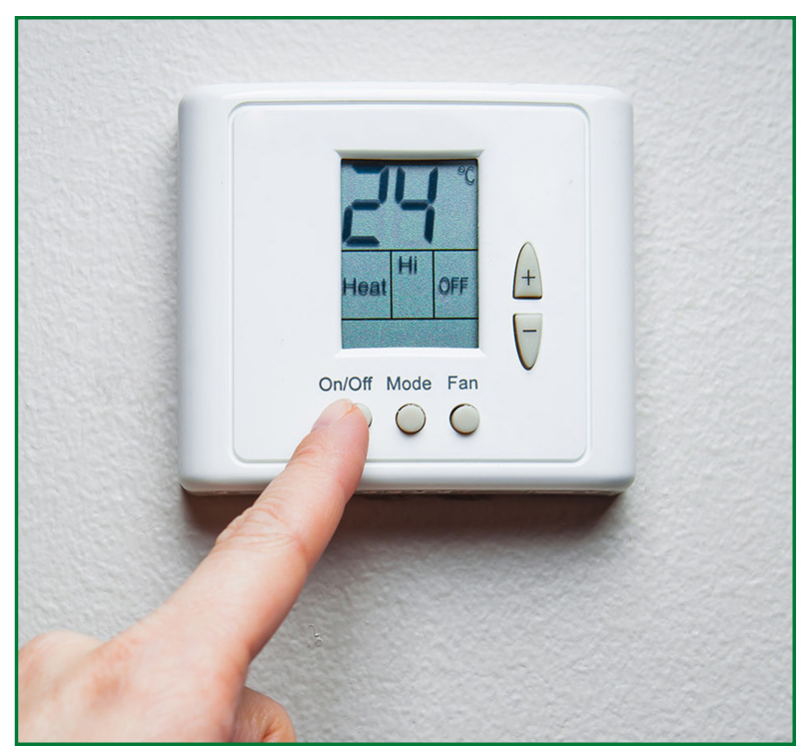

discussed above). All of these capabilities and more are empowering building owners and their operations staff to be more efficient in the use of limited resources and finances while better managing their buildings and trying to improve occupant comfort. These thermostats should have multiple capabilities at the commercial building level, including economizer control, adaptive recovery/ optimal start, outdoor temperature and indoor temperature (wireless) sensing, multiple scheduling capabilities, push-button override, and many others.

Non-programmable thermostats (Figure 9.4) are the simplest thermostats. They allow you to manually change the space temperature set point. Older non-

programmable thermostats sometimes have only one set point. The disadvantage of these thermostats is that if you want the space to be at a different temperature (e.g., on nights, weekends, holidays), you must change the set point every time, manually. 
The abilities of a programmable thermostat (Figure 9.5) vary but most allow for setting different temperature set points for different times of the day and days of the week. The idea is that you use this scheduling capability to configure the HVAC system to more energy-efficient set points (which are usually less comfortable set points) during the times that the space is not occupied. The exact capabilities that a programmable thermostat can perform vary from thermostat to thermostat. Depending upon the thermostat's capabilities, temperatures can be set in three different formats: seperate set points for weekdays and weekends, seperate set points for weekdays plus Saturday and Sunday, or seperate set points for each day of the week. More sophisticated thermostats have four set points: occupied hours heating and cooling set points, plus night/weekend setback heating and cooling set points

The thermostat allows you to define the occupied hours of the building that have a tighter temperature range (for example, $70^{\circ} \mathrm{F}$ to $75^{\circ} \mathrm{F}$ from 6 a.m. to 6 p.m. on weekdays) and the unoccupied hours that have a much wider temperature range-called the setback temperatures (for example, $55^{\circ} \mathrm{F}$ to $85^{\circ} \mathrm{F}$ on nights and weekends). This way the building does not use energy and it maintains a comfortable temperature when unoccupied. Setting back temperatures is a better option than completely shutting down the unit, because it allows for better temperature recovery in the morning.

More sophisticated thermostats have remote push-button override capability. This compensates for an occupant working late or on a weekend (when the thermostat is scheduled for setback values). When the button is pushed, the programmable thermostat can be configured to provide between 1 and 2 hours of conditioned temperatures that match occupied set points.

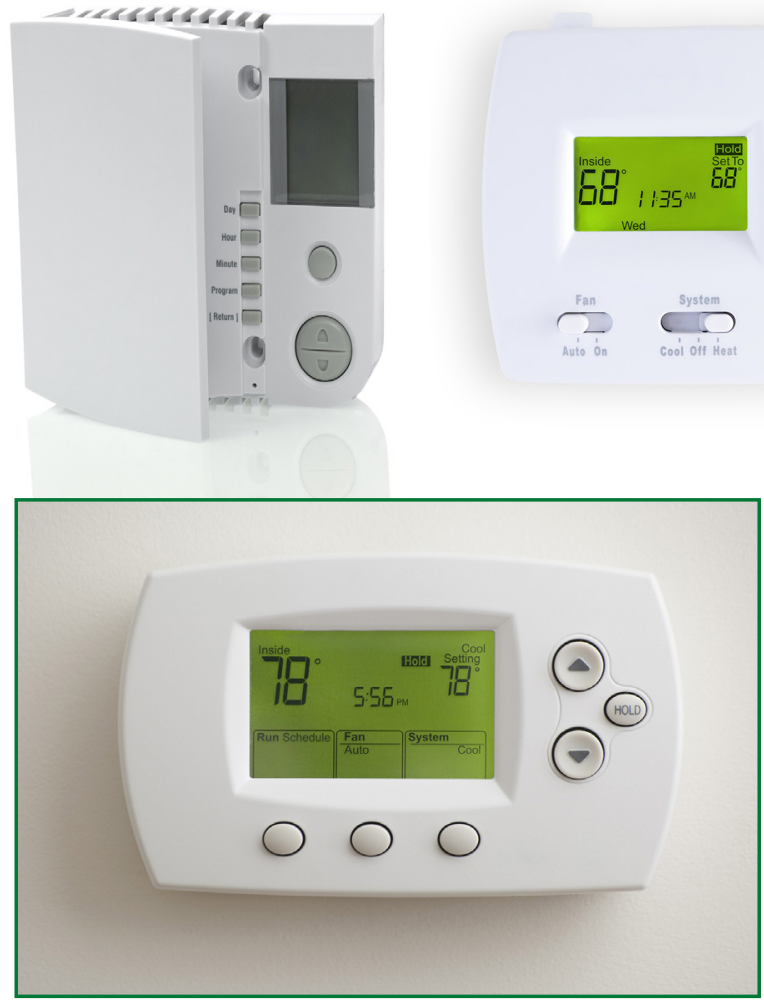

Figure 9.5. Programmable thermostats.
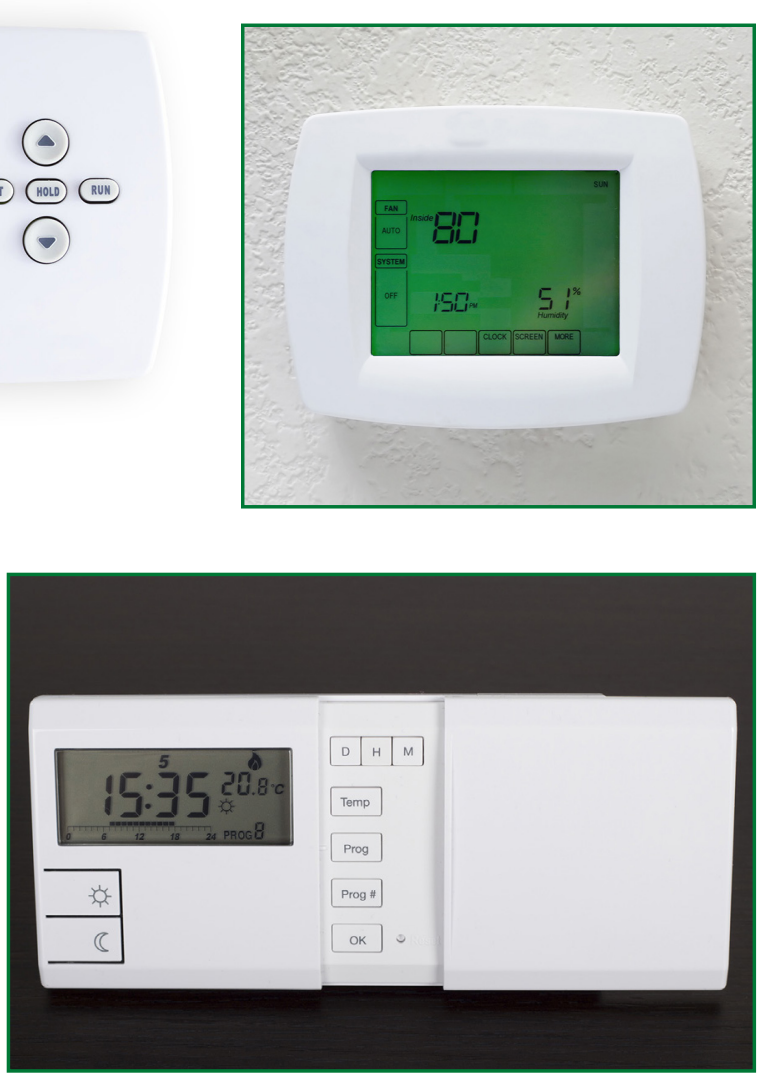
Sophisticated thermostats may have direct control of the economizer dampers and allow for elimination of the existing economizer controller (see discussion below about economizer controllers).

More sophisticated thermostats should also have an optimal start or "intelligent recovery" function that automatically determines the optimum time to activate the HVAC system to return the space temperatures to within 1 to $2^{\circ} \mathrm{F}$ of desired set points, just prior to scheduled occupancy. Over time, the thermostat "learns" the optimum time to prestart when conditions mandate. This saves energy when compared to thermostats that always start up the HVAC system too earlier than necessary.

\subsubsection{Humidity Sensors}

Humidity sensors are used in buildings where humidity (either too high or too low) is a concern. For most buildings, satisfactory dehumidification of warm and humid outdoor and/or return air occurs simply by running the cooling system. As warm, humid air passes over the cooling coil in an air handler or rooftop unit that is actively cooling the air, some of that moisture condenses on the surface of the cooling coil, which removes the excess moisture from the air. A humidity sensor can be used to detect whether the cooling system is adequately reducing the humidity levels in the building or whether it needs to work harder to remove moisture from the air. During dry periods (typically in the winter), a humidity sensor can be used to sense indoor humidity to maintain a minimum level of humidity. When indoor humidity becomes too low, it may trigger a humidifier to come on. In more humid climates and/ or in certain buildings with high sources of internal humidity generation (e.g., from people, showers, cooking equipment, etc.), supplemental humidification may not be necessary.
Some buildings have outdoor air humidity sensors. These are used to control

economizers (called enthalpy economizers) in humid climates because the outdoor air temperature alone may not provide enough information to determine whether free cooling from the outdoor air is beneficial, or whether it will require too much humidity removal from the cooling coil.

\subsubsection{Economizers}

An economizer is composed of a ductand-damper arrangement and automatic control system. It allows a cooling system to supply outdoor air to reduce or eliminate the need for mechanical cooling during mild or cold weather. The economizer adjusts the dampers to admit more outdoor air through the outdoor air intake (Figure 9.6) when "free cooling" is available and reverts to the minimum damper position for ventilation when outdoor air conditions are not optimum.

The simplest control sequence for economizers uses only an outside air temperature sensor to determine when to open the damper and allow in more outdoor air. More complex control sequences use temperature sensors in the outside air and return air to determine the "optimum" air stream for cooling (see " $T$ " labels for temperature sensors in schematic, Figure 9.6).

When the thermostat calls for cooling, the economizer control determines whether the outdoor air temperature is cooler than the return air temperature and adjusts the outdoor and return air damper opening such that the mixed air set point temperature (or the supply air set point temperature) is satisfied. The outside air damper opens to admit as much as 100\% outdoor air. If the outside air damper is open $100 \%$ and the mixed air set point is still not satisfied, then and only then does the cooling coil(s) activate. 
The most complicated economizer control sequence is called differential enthalpy

control. It uses the outdoor air and return air humidity sensors (see "H" labels for humidity sensors in schematic, Figure 9.6), as well as outdoor air and return air temperature sensors to determine which air stream should be used.

Differential enthalpy control is especially useful in humid climates, where hot weather is typically associated with humid conditions. Because humid air may contain too much moisture for the indoor environment and require dehumidification at the cooling coil, the control algorithm needs to determine whether the outdoor air has an enthalpy value (sensible plus latent heat) that is less than the return air enthalpy to initiate economizing using outdoor air. Calculation of the enthalpy is done by the controller and requires inputs from a temperature sensor and humidity sensor in the same air stream. When differential enthalpy is required, four sensors are used and connected to the economizer controller.

\subsubsection{Occupancy Sensors}

Occupancy sensors are used to turn lights and/or appliances and HVAC systems on or off depending on whether someone is in the room. They use a variety of methods from infrared (heat) detection to ultrasonic (motion) waves to detect either heat or movement (or both). They turn on the power to selected appliances when they detect heat or movement and then turn off the power after not detecting heat or movement for a several minutes. The occupancy sensor in Figure 9.7 is a typical ceiling-mounted sensor. The sensor pictured in Figure 9.8 is a typical wall-mounted sensor. The sensors should be mounted where they have the best view of the room and its entrance. Typical applications for ceiling-mounted sensors include bathrooms and large common areas. Typical applications for wall-mounted sensors include office and other similar areas. Newer wall-mounted sensors (called vacancy sensors) are designed to turn off lights, but require the occupants to manually turn the

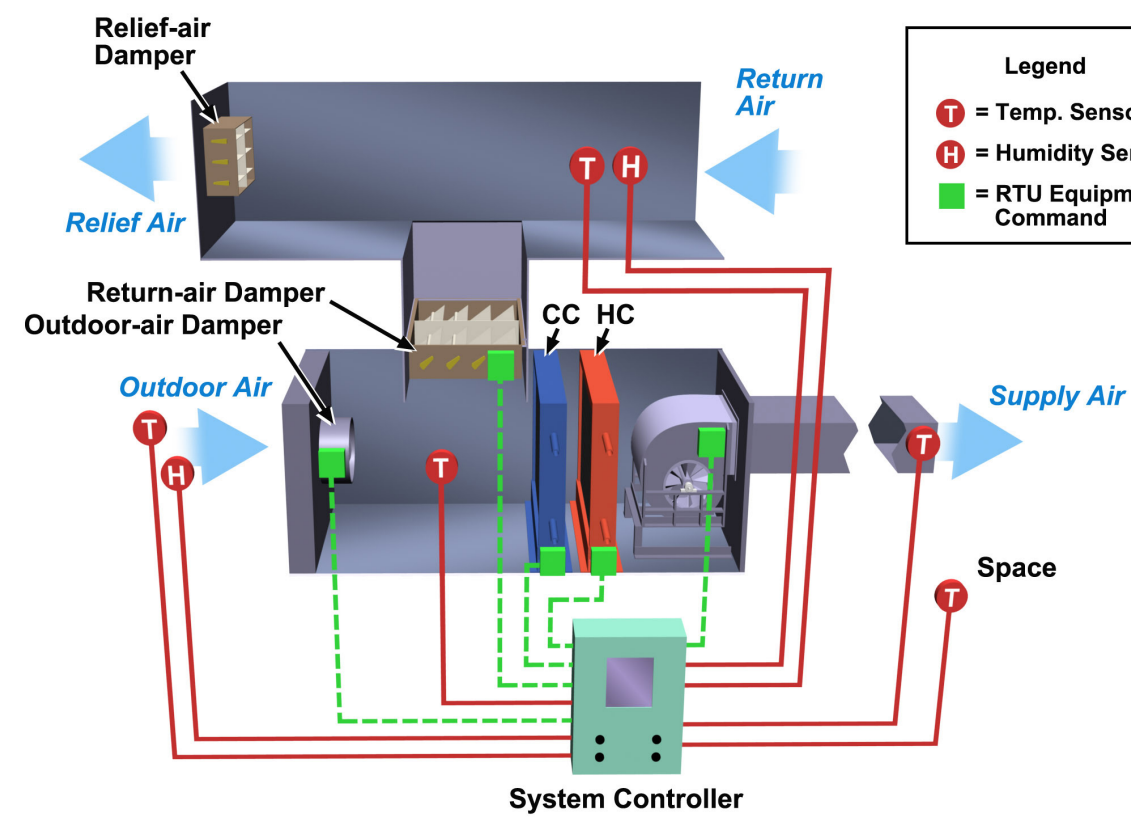

Figure 9.6. Schematic of an economizer unit (left) $(C C=$ cooling coil

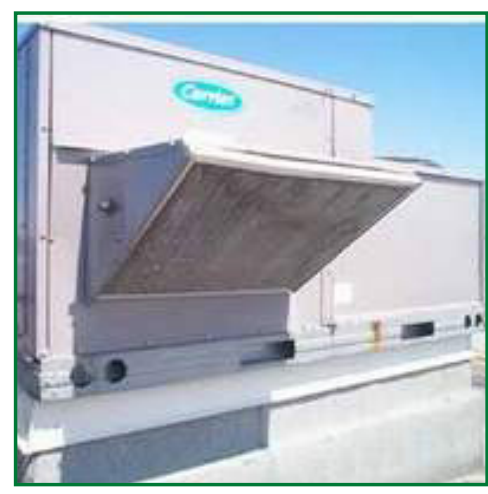
and $\mathrm{HC}=$ heating coil); outdoor air intake for economizer (right). 


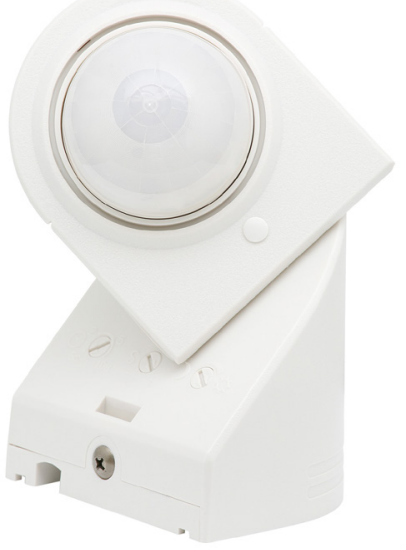

Figure 9.7. Ceiling-mounted occupancy sensor.

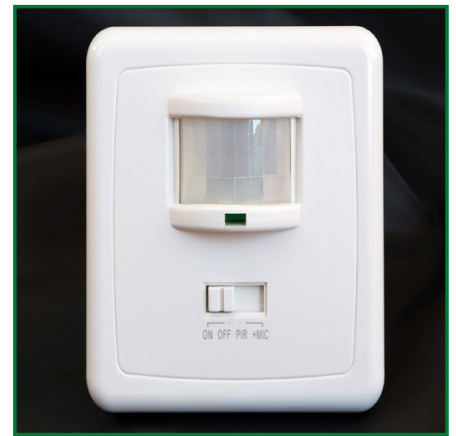

Figure 9.8. Wall-mounted occupancy sensor.

lights back on. This is by design because many wall-mounted sensors are located by door entry points and can "false-trip" the lights on, when people simply walk by without entering the space or momentarily "poke" their heads into the space. This saves more energy and reduces the short-cycling of equipment.

\subsubsection{Light Sensor (Photocell)}

A photocell is a small device used to detect light. Photocell sensors are typically smaller than a nickel and can easily be identified by their signature zigzag pattern (Figure 9.9). When installed indoors, they are used to dim or shut off interior lights based on the amount of ambient sunlight coming in from exterior windows. When installed outdoors, they are typically used as a sensor to tell whether it is day or night and to turn exterior lights on or off. Lighting sensors placed outside to control exterior lights are prone to getting dirty. If they are dirty, they cannot sense the surrounding light and need to be dusted off. When the outdoor lights are on during the day, this is usually an indication that the sensor has become dirty.

\subsubsection{Carbon Dioxide Sensors}

Buildings with "demand control ventilation" (also referred to as DCV) use carbon dioxide sensors to determine the amount of ventilation air derived from the outside air. People in a building are constantly inhaling oxygen and exhaling carbon dioxide. The buildup of carbon dioxide is one of the primary drivers for introducing more outside air ventilation. Demand control ventilation and carbon dioxide sensors are a more advanced form of ventilation control and are not very common at this time.
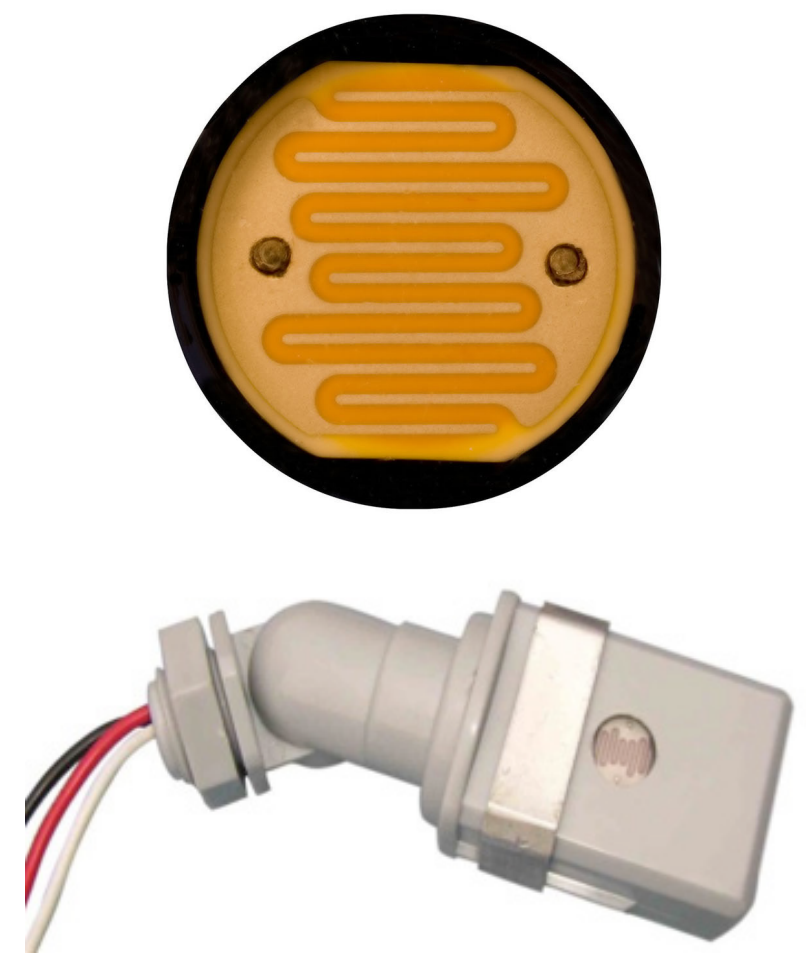

Figure 9.9. Photocell sensors. 


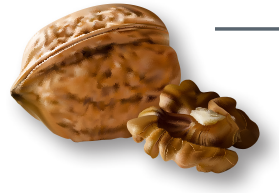

In a nutshell ... Sensors

and control systems are used within the HVAC

system and occasionally within the lighting system and other building systems to provide automatic, smart control of building energy systems. Properly functioning control systems require monitoring to confirm of the integrity of the sensors, the functionality of the actuators, and the use of intelligent set points. Additional sensors may be used for monitoring purposes only. Two common control systems that should be monitored closely to save energy are thermostats and economizers.

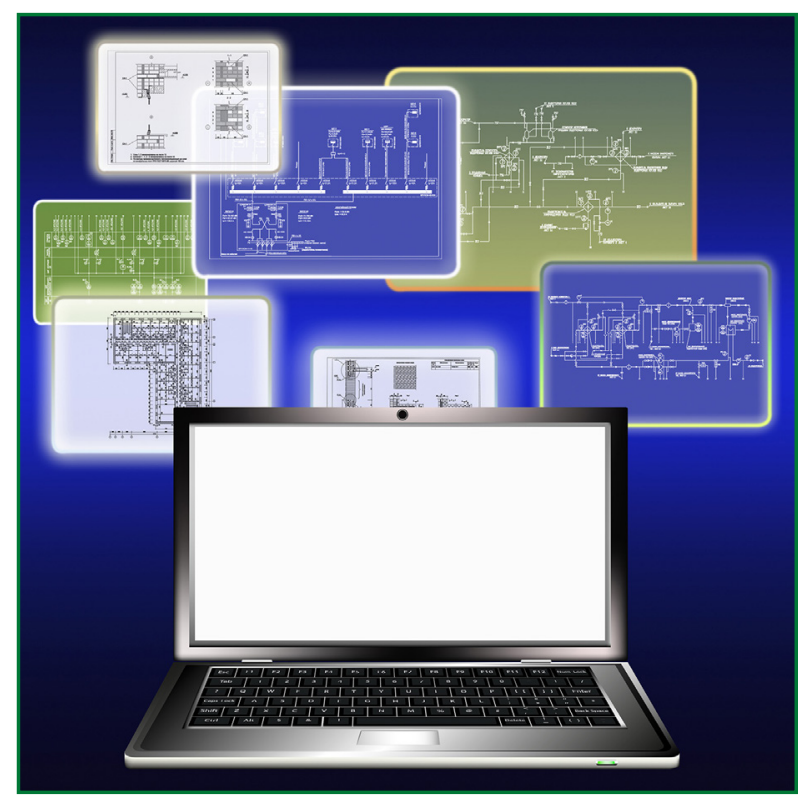

Figure 9.10. Building operation graphic screens.

they arise. O\&M duties may take up only a fraction of one person's time and fall on the shoulders of someone with many other job duties. Improvement of operations, either for dedicated O\&M personnel using a BAS or for managers of small buildings, can have very large impacts on energy consumption.

\subsubsection{Proactive Versus Reactive Management}

Usually the default mode of control for building operators, regardless of the size of the building, is to manage only to the day-to-day concerns of the building and its occupants and respond to problems or "put out fires" as they arise. This is called a reactive management approach. Buildings managed under this approach are typically configured using constant settings for building systems that often get configured when problems arise or when occupants complain. Some lights and HVAC systems may run 24 hours per day, 7 days per week, and thermostats may be set up to use constant set points all the time, which may compete with set points at other nearby 
thermostats. From an O\&M perspective, this approach can be very costly, in terms of energy consumption, reduced equipment life spans, and paying for additional visits by maintenance contractors.

The smart alternative to reactive management is called proactive management. It involves the regular scheduling of inspections of building infrastructure and equipment to manage simultaneously for occupant comfort, reduced energy consumption, and improved equipment performance/life span (Figure 9.11).

Car owners learn the value of proactive car care to prevent wear. They schedule regular inspections, oil changes, and maintenance -changing the oil, replacing timing belts before they break and catastrophically harm the engine, and replacing tires before they explode on the highway. These are all proactive approaches that most of us follow because they are proven to reduce long-term costs associated with car ownership.
Proactive maintenance for a small building may include this sample list of tasks:

\section{- Periodically inspect packaged rooftop}

units. Replace filters found to be heavily loaded with dust/debris. Clean coils found to be coated with dust/dirt. Check outdoor air dampers to make sure they are working properly and are not blocked.

- Periodically inspect the envelope. Make sure windows, entrance doors, and rollup doors are closed when it is cold or hot outside. Check weather-stripping seals on doors to make sure they are intact. Check the attic/roof to make sure insulation is intact/has not moved and there is no infiltration of moisture/moisture damage.

- Periodically evaluate thermostats. Confirm that thermostats are set to only condition space during occupied periods nearby thermostats have reasonably consistent set points, and thermostats appear to be reading reasonable temperatures.

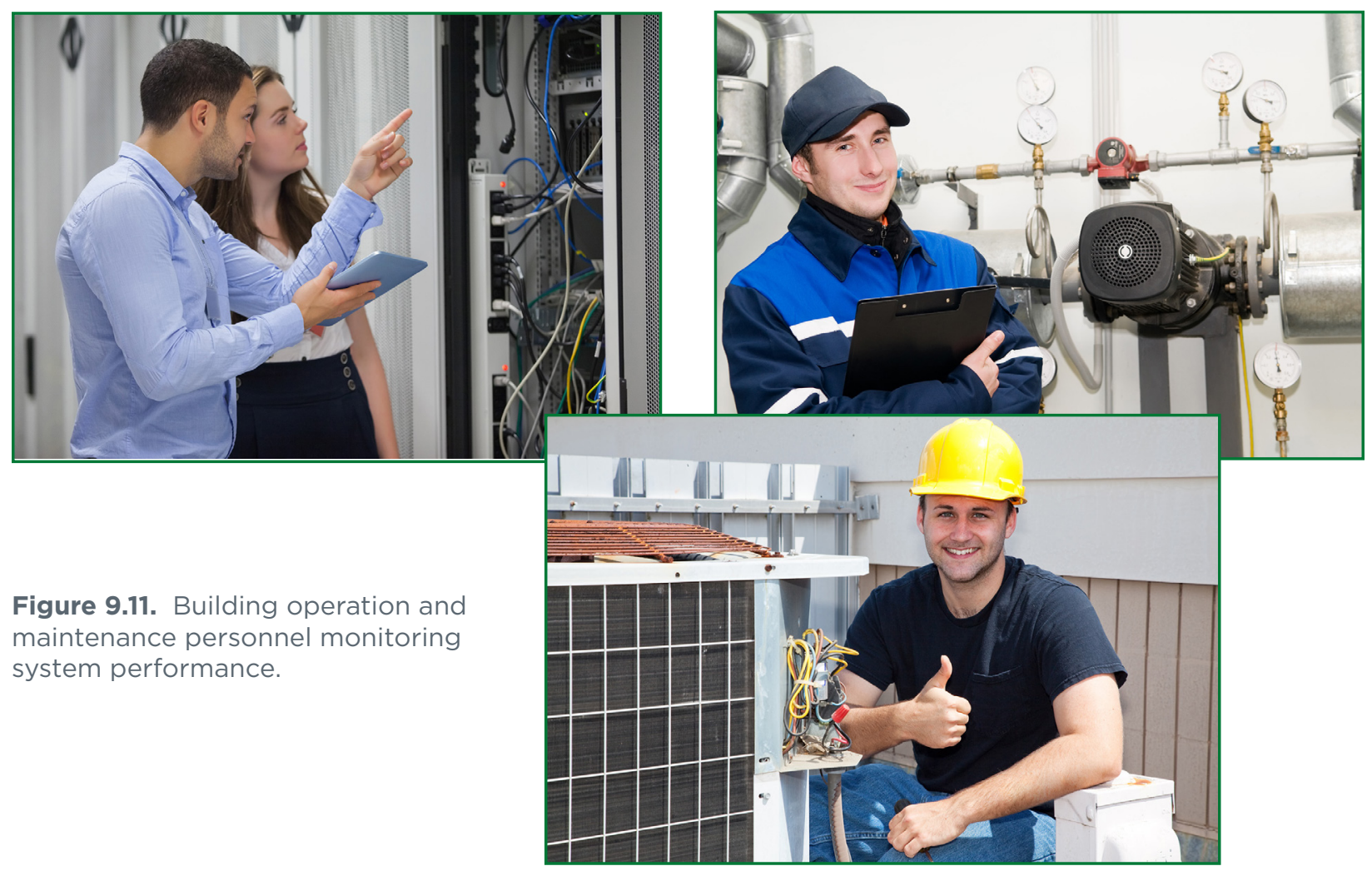


- Talk to occupants. Are they comfortable; do they notice any operational problems or see opportunities for improved energy efficiency?

- Periodically review lighting control. Make sure lights on timing circuits are set to proper schedules and that they are being turned off as intended. Check the functionality of any daylight sensors and occupancy sensors. Check for lamps that have burned out and replace them. Look for spaces that are over-lit and de-lamp as necessary.

For building equipment and lighting, most devices have an expected lifetime that is based on the number of runtime hours at recommended conditions. Reducing the runtime hours and providing the recommended conditions can greatly extend the life of equipment.

\subsubsection{Morning Warm-Up and Cool-Down}

A proactive approach to HVAC operations involves using the thermostat's unoccupied mode for nights and weekends (or any period that the space served is not occupied). For small buildings controlled by thermostats, this involves using a programmable thermostat that widens the temperature settings during unoccupied periods (typically nights and weekends). Larger buildings use an unoccupied mode to control nighttime fan operation and may even lock out boilers and chillers overnight. When the building goes from occupied mode to unoccupied mode, typically the HVAC system shuts off for a period of hours (or potentially all night or all weekend during mild weather) and the temperatures "floats" up or down toward the unoccupied settings.
In the morning, it usually takes a varying period of time for the building space temperatures to recover to the desired occupancy temperatures prior to the arrival of occupants. This varying time period is called the morning warm-up (in the case of heating season operation), or the morning cool-down (in the case of cooling season operation). Because this variable time period is required to help the building recover to the desired comfort levels in advance of occupancy, many building operators have learned to schedule the HVAC system and/ or the thermostats to return to the occupied mode one-half hour to several hours before the scheduled occupancy period begins. In most cases, this time period is determined by building operators, based on the worst-case scenario. It is often determined during the most frigid or the hottest and most muggy mornings of the year.

For small buildings, an opportunity for energy savings called adaptive recovery is available on many newer thermostats. Adaptive recovery (also called "optimal start") is designed to keep the building in the unoccupied mode for as long as possible. Adaptive recovery allows the user to configure the thermostat so it automatically adjusts (adapts) the time needed to warm up or cool down the space served by the HVAC system to ensure the space temperatures return to close to the desired occupied conditions just before the occupancy period begins. Over time the thermostat "learns" how long it takes the HVAC system to warm up or cool down the space and it automatically adjusts the warm-up or cooldown time period required to achieve the desired occupied set points. 


\subsection{BASIC TOOLS NEEDED FOR RETUNING}

Tools that are helpful during the walkdown of a building for re-tuning include common workbench tools (screwdrivers, allen wrenches, adjustable wrenches, tape measure) as well as other specialty tools such as a light meter, magnetic ballast detector "top," infrared (IR) temperature gun, wind velocity meter, and IR imaging camera. These tools are shown in Figure 10.1.

Workbench tools are helpful to carry around for opening access panels, tightening loose connectors, and taking measurements. As described in the lighting section, a light meter is used during a building walk-down to determine if areas are under-lit or over-lit. Target light levels for key areas include 37 foot-candles for office areas, 28 foot-candles in bathrooms, and 5 foot-candles in corridors (see Section 7.0). A magnetic ballast detector is used to determine whether fluorescent

lighting systems are using older, inefficient magnetic ballasts or newer electronic ballasts. The detector is designed to be spun like a child's toy "top." If the pattern on top appears smooth when the top is spun, the ballasts are electronic. If the pattern on top has a pulsed pattern visual effect, the ballast is magnetic. Wind velocity meters are inexpensive hand-held devices with a small, built-in fan rotor that measures the speed of a flowing air stream. Such meters can be used to measure and verify the speed of air at various places in the HVAC system (outdoor, relief air, terminal box discharge) and to calculate estimated flow rates, when duct cross-sectional area is taken into account. An IR temperature gun is used to measure the temperature at a specific point, using a laser pointer as a guide. It is good for taking spot measurements of temperatures

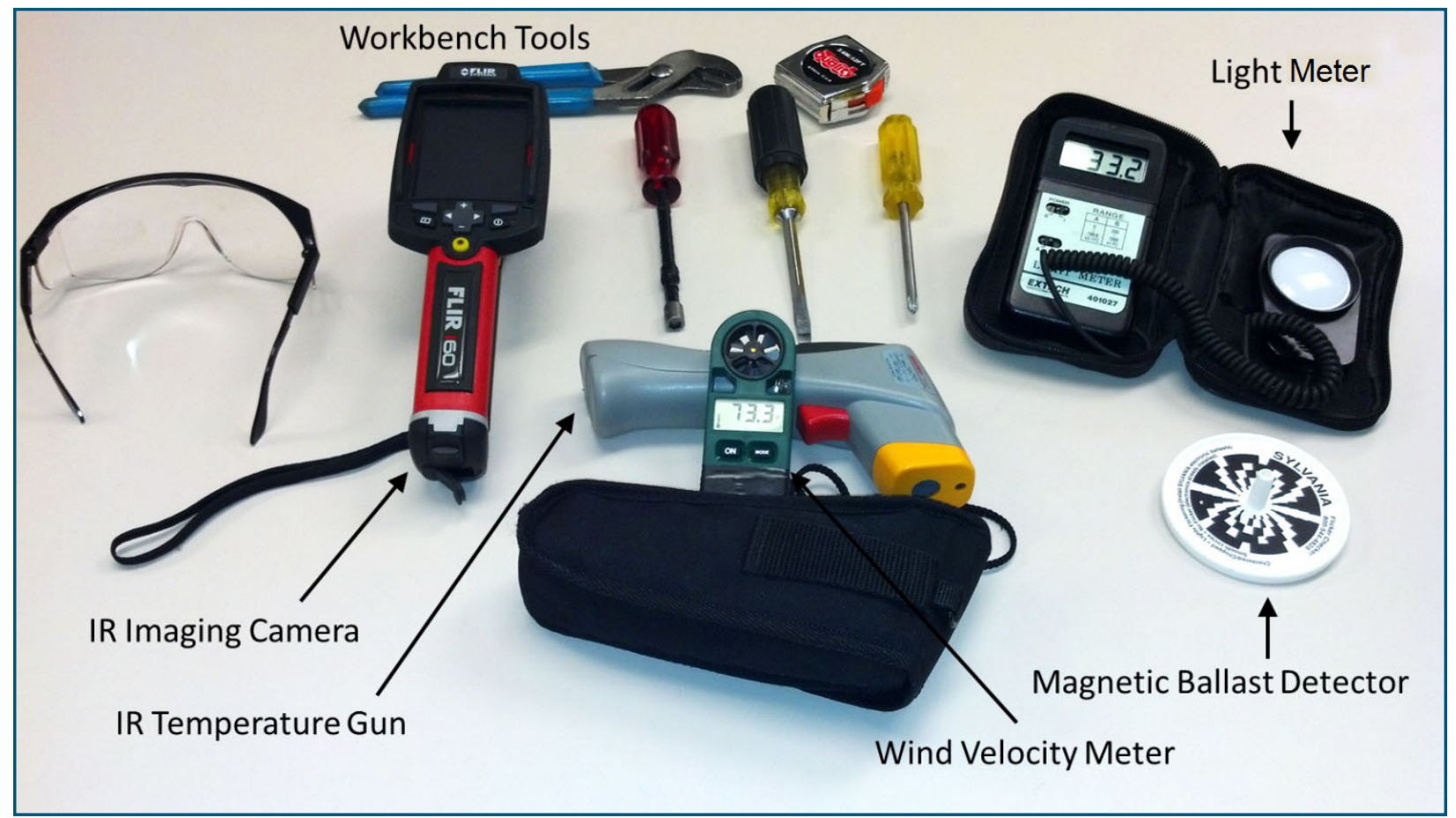

Figure 10.1. Tools for a building walk-down. 
Small Commercial Building Re-tuning: A Primer

on HVAC ductwork and piping, as well as

taking indoor zone and outdoor temperature readings. An IR imaging camera is a more expensive device that reproduces an image of an area, color-coded by temperature. This kind of image is good for use on the building envelope to identify areas of outdoor air infiltration or wall sections without insulation, and on ductwork to identify uninsulated or leaking areas. 


\subsection{CONCLUSION}

This primer on building energy was developed by DOE to help building owners, building managers, and their operations staff better understand building energy fundamentals. More specific and detailed information about building energy can be found at the DOE website

(www.energy.gov), with information directed specifically toward buildings found on the Energy Saver website (http://energy.gov/energysaver/energy-saver) and the Building Technologies Office website (http://www1.eere.energy.gov/buildings/). This document is intended to be a primer for DOE's small commercial building re-tuning training curriculum, which is a training course that helps small commercial building owners and operators identify inefficient operations, detect energy savings opportunities, and implement improvements.

By learning some basic terminology and fundamental concepts related to the building exterior; building interior; HVAC systems; lighting; water-heating systems; meters, sensors and controls; and re-tuning tools readers should be "primed" to learn more about re-tuning opportunities and identify and correct building energy operational issues at minimal cost to the building owner. 
Small Commercial Building Re-tuning: A Primer 


\section{APPENDIX A: GLOSSARY OF TERMS}

absolute humidity - The quantity of water in a particular volume of air.

actuator - A mechanical device (usually a motor) for moving or controlling something (such as a damper or valve).

adaptive recovery - A feature of a thermostat where it automatically adjusts (adapts) the time needed to warm up or cool down the space served by the HVAC system to ensure the space temperatures return to close to the desired occupied conditions (from a set back period) just before the occupancy period begins. This is sometimes referred to as an "optimal start" setting.

air balance - A term used in the HVAC field to describe the distribution of air through an HVAC system to match the amount needed. "Air balance" or "air balancing" is also the process of testing a HVAC system to make sure it is delivering the desired amounts of air to different rooms.

air distribution system - A system that uses a duct and fan arrangement to deliver air throughout a building.

air intake - An opening or inlet through which outside air is brought into an HVAC system.

air-side economizer - see economizer

ambient - Refers to the immediate surroundings; thus, the ambient temperature is the temperature of the surrounding air.

argon gas - Colorless, odorless gas used in the air space of double-pane low-E glass to increase the insulating performance.

auxiliary heating - Extra heating (like electric heating or gas furnace) that turns on when it is too cold for a heat pump to operate.

backflow - A damper with gravity-weighted damper blades designed to allow airflow in one direction. This is sometimes referred to as the back-draft damper.

ballast - An electrical device for starting and regulating fluorescent and discharge lamps.

Btu (British thermal unit) - A measurement of energy representing the amount of heat needed to raise 1 pound of water by $1^{\circ}$ Fahrenheit; approximately the same amount of energy released by a single lit match.

building automation system (BAS) - A system that optimizes the startup and performance of the heating and cooling (HVAC) equipment and alarm systems. A BAS greatly increases the interaction of mechanical subsystems within a building, improves occupant comfort, lowers energy use, and allows offsite building control.

building envelope - This is the physical separation between the inside and the outside of a building. It includes things like the insulation in the walls and windows. It is also referred to as the building shell.

building operations - The manner by which a building is configured, operated, and maintained to meet the occupants' needs.

building re-tuning - A systematic process of identifying and correcting building operational problems that lead to energy waste and improve a building's energy efficiency through lowcost and no-cost operational improvements. Re-tuning usually consists of checking a building for inefficiencies ( like lights being left on when not needed or deficiencies in a building's thermal envelope) and a thorough check of the heating and cooling (HVAC) systems (also see: energy auditing)

caulk or caulking - Caulk is a waterproof filler and sealant used to seal joints. In a building, caulk is typically used and visible around window frames and around any plumbing and piping penetrations of outside walls.. 
CFL - This is short for "compact fluorescent lamp." It is the same thing as a regular fluorescent light, but is meant to fit in a regular incandescent light socket. CFLs are more efficient than a standard incandescent light bulb.

chiller - The term "chiller" usually refers to a chilled-water applied system, which uses chilled water to transport heat energy between the air-side, chillers, and outdoors. These systems are commonly found in large HVAC installations. Most commercial buildings are cooled with either DX systems or chillers.

coefficient of performance (COP) - The ratio of heating or cooling provided by a heat pump over the electrical energy consumed.

compressor - A device used to compress refrigerant gas in cooling systems or compress air for building HVAC systems that require pressurized air to move valves or dampers (or thermostat controls to function).

condenser - The part of a heat pump or air conditioner that condenses the refrigerant vapor to a liquid.

conditioned space - The space within a building provided with heated or cooled air, or both, and where required, with means of humidification or dehumidification to maintain conditions for an acceptable thermal environment.

\section{control systems - See building automation system}

cool roof - A cool roof is one that is made with a reflective surface that can deliver high solar reflectance, reducing heat transfer to the surface, and high thermal emittance (the ability to radiate absorbed, or non-reflected solar energy).

cooling coil (also known as an evaporator coil) - A coiled arrangement of pipe or tubing that allows the air stream to be cooled as it moves across the coil. As the air moves across the coil, heat exchange occurs as heat is gained through the surface area of the coil, making the air stream colder.. The most common heat exchange mediums are water, a water-glycol solution, or a refrigerant. When a refrigerant is used in the system (in the case of direct expansion/DX or direct refrigeration systems), the cooling coil is referred to as the evaporator coil.

cooling load - The amount of cooling required to keep a building at a specified temperature (e.g., maintain $78^{\circ} \mathrm{F}$ ) during the summer/warmer months, regardless of outside temperature.

cooling set point - The cooling set point on a thermostat is the temperature setting that initiates the cooling system. A typical cooling set point is around $78^{\circ} \mathrm{F}$, such that the thermostat initiates cooling above this temperature to maintain the building temperature near this set point.

cycling - Describes the process when the heating and cooling system is oscillating back and forth between on and off (also referred to as "short-cycling"). This is a very inefficient way to condition a building.

damper - A device that can change how much air is allowed to pass downstream of the device in a fan and duct configuration. It is used in a heating and cooling (HVAC) system to direct air and change the amount of air the system derives from or delivers to different places.

dead band - The dead band control for thermostats typically consists of a 2 to $10^{\circ} \mathrm{F}$ "band" on a thermostat that eliminates the possibility of short cycling between heating and cooling or cycling rapidly back and forth between heating and cooling. When the temperatures fall within the set dead band zone, neither heating nor cooling occurs.

desuperheater - A device that reduces the temperature of steam or refrigerant vapor and recovers useful heat in the process.

dew point - The temperature to which air must be cooled, at a given pressure and water-vapor content, for it to reach saturation; the temperature at which dew begins to form. 
diffuser - The ends on ventilation ducts that are shaped to deliver air in one or more directions. Generally, a diffuser discharges air in a spreading stream. Diffusers can be located on either the floor or the ceiling. They are also called grills or registers. The term "diffuser" is also used in conjunction with lighting and light fixtures to describe the translucent material used for filtering glare from a light source. In this context, it is also sometimes referred to as a reflector.

direct expansion (DX) - A finned coil, used in air cooling systems, inside of which circulates a evaporating refrigerant.

direct expansion (DX) system - In a direct-expansion unit, the evaporator is in direct contact with the air stream so the cooling coil of the air-side loop is also the evaporator of the refrigeration loop. The term "direct" refers to the position of the evaporator with respect to the air-side loop. The term "expansion" refers to the method used to introduce the refrigerant into the cooling coil. Most commercial buildings are cooled with either DX units or chilled water systems.

door sweep - A flexible weather stripping used at the top and bottom edges of swinging and revolving doors.

drain pan - A pan (also referred to as a condensate pan) used to catch and collect condensate in a cooling system. The condensate is then removed from the cooling system through a drain line.

dry-bulb temperature - The temperature of air measured by a thermometer freely exposed to the air but shielded from radiation and moisture.

ducts - A cylindrical or rectangular tube used to move air either from exhaust or intake, and for distributing conditioned air to rooms from a conditioning device or providing a means to return air to the distribution fan and the conditioning device.

economizer (also air-side economizer) - A duct-and-damper arrangement coupled with a control system that allows a cooling system to supply outdoor air to reduce or eliminate the need for mechanical cooling when the weather is mild or cold.

electric heat tracing - A system used to maintain or raise the temperature above freezing. These systems are commonly used on piping, water drainage systems and vessels by using an electrical heating element that is in physical contact along the length the device that needs to be protected from freezing.

EnergyStar label - The Energy Star Label indicates that the product is relatively energyefficient. The labeling convention was developed and is currently regulated by the U.S. Environmental Protection Agency and the U.S. Department of Energy.

enthalpy - A measure of the total heat content of air, combining the concepts of sensible heat and latent heat into one measurement.

enthalpy control - Controlling the economizer based on a measurement of enthalpy, or total energy content of the air, not its temperature. This makes a difference when the outdoor air is humid compared to the indoor air.

evaporator coil - See cooling/heating coil.

fenestration - The opening in the surface of a building structure, such as a window, door, or skylight.

fixture - When related to lighting, a fixture refers to a complete lighting unit consisting of a lamp (e.g., fluorescent tube, CFL, incandescent bulb, etc.) matched with the driver or ballast if needed, something that distributes and protects the light, as well as the components needed to connect the unit to an electric circuit.

flashing - A weather-proof material, such as sheet metal, used to reinforce and weatherproof the joints and angles of a roof. It is also found around sewer vents, flue pipes, and other penetrations that come up or go down through the roof. 
fluorescent lighting - A lamp that produces visible light by fluorescence, commonly a glass tube whose inner wall is coated with a material that fluoresces when an electrical current causes a vapor within the tube to discharge electrons.

footprint - A building's footprint is the property area that is to be covered by the building.

gas furnace - A furnace that burns gas for heat.

grill - see diffuser or register

heat pump - A device that transfers heat from a colder area to a hotter area by using mechanical energy. Heat pumps can be used for heating or cooling a building by transferring heat through mechanical means either to or from an external source (such as the ground, water, or outside air). The direction of the heat movement determines whether the building is heated or cooled. Heat pumps can also be used to heat water as part of a water-heating system.

\section{heat trace - See electric heat trace.}

heating coil - A coiled arrangement of pipe or tubing that allows the air stream to be heated as it moves across the coil. As the air moves across the coil, heat exchange occurs as heat is lost through the surface area of the coil, making the air stream hotter. Depending on the system, the most common heat exchange mediums are water, a water-glycol solution, steam or a refrigerant (heat pump systems).

heating load - The amount of heating required to keep a building at a specified temperature (e.g., maintain $65^{\circ} \mathrm{F}$ ) during the cool-weather months, regardless of outside temperature.

heating seasonal performance factor (HSPF) - A measurement of efficiency of air-source heat pumps, measured in BTU/Watt-hours. The higher the HSPF rating of a unit, the more energy efficient it is.

heating setpoint - The heating set point on a thermostat is the temperature setting that activates the heating system. A typical heating set point is around $68^{\circ} \mathrm{F}$, such that the thermostat activates the heating system below this temperature to maintain the building temperature near this set point.

high intensity discharge (HID) - HID lamps are the type of electrical gas-discharge lamps that produce light by means of an electric arc between tungsten electrodes housed inside a translucent fused arc tube. HIDlamps provide more visible light per unit of electric power consumed than fluorescent and incandescent lamps because a greater proportion of their radiation is visible light in contrast to heat. Examples include metal-halide lamps, mercury vapor lamps, and high-pressure sodium lamps.

high temperature sink -An environment or protective device that is able to absorb heat without a phase change or without appreciably changing the temperature.

hot-water tank - A device used to store and heat hot water. The hot water is used for domestic (human consumption) or comfort heating applications.

hot-water system - The system that heats then distributes water in a building. This system includes the hot-water tank(s) and the pumping and piping conveyances used to distribute the heated water throughout a building.

HVAC - Abbreviation for "heating, ventilation, and air conditioning." It refers to the system that is used to provide heating and cooling services to a building.

illumination - The amount of light provided by a lamp and/or the brightness of this light.

incandescent lamp - A lamp that creates light by heating up a filament of wire. The filament is located inside a bulb and an electric current passes through the filament, causing incandescence. The glass bulb containing the filament is filled with a nonreactive gas, such as argon, to prevent the wire from burning. 
infiltration - In building terms, infiltration is the uncontrolled flow of air or water into and out of a building through cracks, openings, doors, or other areas that allow air to move. In a building, air infiltration is a major factor in energy efficiency and water infiltration is a major factor in structural damage and mold formation.

internal heat gain - All sources of heat that come from inside of a building, including occupants, computers and other equipment, and lighting. Heat gain can also come about through external sources, such as solar heat gain.

instantaneous water heater - A water heater located at the "point-of-use" that heats water only when a hot water valve is opened. Sometime these water heaters are also referred to as on-demand or tankless water heaters.

insulation - Material that prevents or reduces the passage or transfer of heat or sound. In terms of building energy, it is typically thermal insulation, which is designed to reduce unwanted heat loss or gain from a building and can decrease the energy demands of heating and cooling systems.

latent heat - The heat required to convert a solid into a liquid or vapor, or a liquid into a vapor, without change of temperature. Latent heat is sometimes referred to as "hidden heat" because it cannot be measured with a thermometer.

life-cycle cost - A measurement of the total cost of using equipment over the entire lifetime of the equipment (it includes the initial cost, cost to maintain it over its life and the cost of energy it uses).

light emitting diode (LED) - An LED is a semiconductor light source.

light meter - A device used to measure the amount of radiant light energy in terms of lumen intensity.

lighting diffuser - A component of a lighting fixture consisting of a translucent or frosted covering that is used to diffuse or scatter the light and prevent glare.

load profile - How the demand for energy services within a building varies over time (e.g., day, week, month, or year).

low-emissivity (or "low-E") coating -A coated glass product that primarily functions to suppress or filter out part of the light spectrum that transmits heat. In the summer months, the low-e glass filters long-wave radiation from the sun, which reduces solar heat gain in a building (and reduces cooling requirements). In the winter months, low-e glass allows warm solar rays into a building, while suppressing heat transmission out of the building, thereby keeping the building warmer. It is used on windows to provide greater energy efficiency and protection from ultraviolet rays entering buildings.

Iumen - The lumen unit of luminous flux is a unit of measurement that measures the total amount of visible light emitted by a source.

luminous efficacy - A measure of how well a light source produces visible light. It is the ratio of luminous flux to power. It is typically measured in lumens/watt.

meter profile - How the energy usage (or energy consumption) of a building varies throughout atime period (derived from looking at the energy usage from its meter).

meters - A sensor that is used to measure a consumption (water, electricity or gas).

occupancy sensor - A device that turns appliances (typically lighting) on or off depending on whether it detects someone in a room.

on-demand water heating - See instantaneous water heater

packaged system or unit - A heating and cooling (HVAC) system where all the system components required for heating, cooling, and moving air come in one unit. 
photocell - A device that detects light. It is usually used to turn lights on or off depending on whether it is day or night.

plug loads - The electricity use of anything in a building that is powered by an ordinary plug-in outlet (e.g., 120-volt AC outlet). It is sometimes referred to as "miscellaneous electric load," and typically includes things like personal computers, printers, and other office equipment, while excluding all other major end uses in a building such as HVAC, lighting, and water heating.

plenum - A chamber or container for moving air under a slight positive or negative pressure to which one or more ducts are connected. It is sometimes referred to as "plenum chamber."

proactive management - A maintenance management practice that acts in anticipation of future problems, needs, or changes as opposed to reactive, which implies acting after something has already happened.

p-trap - Downward-facing $U$-shaped pipe that traps water in the bottom of the $U$, making an airtight seal that water can pass through.

reflector - A piece of material that reflects light in a required direction.

refrigerant - A substance that remains a gas at low temperatures and pressures and can be used to transfer heat. Freon is an example; it is used in air-conditioning systems.

refrigeration - A system used to cool air by moving heat from one location to another. A refrigeration system may be used within an air-conditioning system to cool a building or it may be used to cool an enclosed refrigerator or freezer.

register - The ends on ventilation ducts that are shaped to deliver air in one or more directions. Generally, a register discharges the air in a confined stream. They can be located on either the floor or the ceiling. They are also called grills or diffusers.

relative humidity - The amount of water vapor actually in the air divided by the amount of water vapor the air can hold (moisture-holding capacity).

return air - Air coming from the building into the heating and cooling system (HVAC system). It may also refer to the return path that the air takes to get to an HVAC system.

roof curb - A small curb put around objects that stick out from the roof like skylights or HVAC systems; it offers a little bit of protection for them and enables the roof structure to properly carry the load while maintaining a moisture barrier (flashing) when installed correctly.

roof deck - The solid material (e.g., plywood or pre-cast concrete) that covers the rafters and provides a surface for the attachment of the outer layer of roofing material.

R-value - A measure of the capacity of a material, such as insulation, to impede heat flow. Higher R-values indicate a greater capacity to impede heat flow. In simple terms, the R-value is considered the inverse or mathematical reciprocal of the "U-factor" or " $U$-value" (i.e., $U=$ 1/R); the $U$-factor is a measurement of heat flow or transmittance. As the R-value goes up, the $U$-value goes down and vice versa. R-values tend to be more commonly used when discussing opaque envelope assembly components, such as insulation, sheathing, interior drywall, etc. and they can be added together to get the total wall/roof/floor R-value. The U-factor, on the other hand, is commonly used to describe the thermal performance of a system, such as a window.

seasonal energy efficiency ratio (SEER) - The cooling output during a typical cooling season divided by the total electric energy input during the same period. The higher the SEER rating for an air-conditioning unit, the more energy efficient it is.

sensible heat - Thermal energy whose transfer to or from a substance results in a change of temperature. The two types of heat energy are "latent" and "sensible" heat where latent heat is related to changes in phase between liquids, gases, and solids, and sensible heat is related to changes in temperature of an object or gas with no change in phase. Sensible heat can be measured with a thermometer. 
sensor - A device that detects or measures a physical property and records, indicates, or otherwise responds to it.

setback- A more energy-efficient temperature setting for the heating and cooling (HVAC) system that would be used when a zone is unoccupied or lightly occupied.

single-, double- and triple-pane windows - Windows with either single or multiple layers of glass separated by a gas. The more layers the window has, the more insulation value it has.

solar collector - Absorption devices that absorb and concentrate solar radiation for the heating of water or buildings.

solar heat gain - Added heat gain from the sun's light rays that come into the building. split HVAC or split system - A heating and cooling system where the components (the condenser and the evaporator coil) are in separate units rather than being contained in the same unit. The condensing unit is located outside the building and the evaporator is located inside the building.

Surface coating - A substance applied to other materials (roofs, walls, floors, etc.) to change the surface properties, such as color, gloss, resistance to wear or chemical attack, or permeability. Surface coatings include such materials as paints, varnishes, enamels, oils, greases, waxes, concrete, lacquers, powder coatings, metal coatings, and fire-retardant formulations.

supply air - The part of a cooling/heating system that supplies the conditioned air from the air-handling unit or furnace to the building space.

T-12, T-8, and T-5 bulbs - These are different formats of fluorescent lights. The smaller number indicates a smaller bulb. They are more efficient than the standard light bulb. T-12 lamps are 11/2 inch diameter, T-8 lamps are 1 inches in diameter and T-5 lamps are 5/8 inch in diameter..

tankless water heating - see instantaneous water heater.

thermal barrier -The insulating barrier on a building that keeps its internal temperature separate from the temperature outside.

thermostat - A device that both lets you set the temperature of a building and acts as a temperature sensor for the air. It can also be used to set the temperature of water as part of a water-heating system.

analog thermostat - A thermostat that uses mercury or alcohol to measure temperature. These thermostats usually are less high tech and do not have an electronic display.

mechanical thermostat -A thermostat without any high-end technology. It only allows you to set the temperature locally at the thermostat via some dial or mechanical adjustment.

non-programmable thermostat -A thermostat that only allows you to set the temperature, usually with a keypad or slide switch adjustment locally at the thermostat

programmable thermostat -A thermostat that allows you to program different heating and cooling settings for different times of the day and week, usually with a keypad locally at the thermostat or remotely from a computer or smart phone with connection to the thermostat.

U-factor - A U-factor or $U$-value is a measure of heat loss in a building element such as a wall, floor, or roof. The higher the $U$-factor, the worse the thermal performance of the building envelope (i.e., roof, wall, or window). A low $U$-factor value usually indicates high levels of insulation. In simple terms, the $U$-factor is the inverse or mathematical reciprocal of the R-value (i.e., $R=1 / U$ ); the R-value measures resistance to heat transfer. The R-values tend to be more commonly used when discussing wall, roof, and floor insulation, while the $U$-factor is commonly used when discussing window-insulating properties. 
ventilation - The process of bringing fresh, filtered air into a building.

vestibule - An enclosed, double entryway into a structure.

weatherized - A weatherized building or building component (e.g., window or door) has had additional protection in the form of insulation or barriers added to reduce infiltration and conductive heat flow into and out of the building. Typical weatherization measures include weather stripping around doors and windows.

weather stripping - Weather stripping can refer to either the process or the agent that is used to seal small openings around windows and doors. Essentially weather stripping is designed to eliminate a flow or draft from an interior space to an exterior space. For drafts coming from a small open space between the bottom of a door and the floor, the addition of a small rubber strip along the threshold will often be sufficient to seal the area. Weather stripping also comes as a roll of foam with an adhesive backing, which can be added around the sides of windows and doors. Weather stripping effectively blocks the air flow between inside and outside, while still allowing the door to be opened and closed with ease.

web-enabled thermostat - a thermostat that is both programmable and remotely accessible, via Ethernet (wired or wireless) communications

wet-bulb temperature - The temperature that a portion of air can be cooled to through addition of moisture to $100 \%$ relative humidity. The web-bulb temperature is always lower than the dry-bulb temperature, but it is identical when $100 \%$ relative humidity is reached (saturation point).

window-to-wall ratio - The ratio of the total area of a building facade that is occupied by windows (glass area and frame).

zoning - Having a heating and cooling (HVAC) system designed to serve different parts of a building. This can be achieved by having dedicated HVAC units serve different sections of a building. Zoning can occur either by floor or by geographical location within a building. Commercial buildings are typically separated into interior zones and perimeter zones where a building is designed with multiple orientations (north, east, south and west). 


\section{APPENDIX B: MORE DETAIL ON HVAC SYSTEM TERMS, DEFINITIONS, AND EFFICIENCY MEASUREMENTS}

For a more advanced understanding of HVAC systems and their controls, there are a few measures that combine temperature and humidity. It is helpful to have a basic understanding of the following terms in order to understand the purpose and results of these efficiency measurements.

- Enthalpy is a measure of the total heat content of the air, combining the concepts of sensible heat and latent heat. It is used to understand the energy impact of bringing unconditioned air into the American Society of Heating, Refrigeration and Air Conditioning (ASHRAE) indoor comfort zone.

- The dew point of air is the temperature at which moisture will condense out of air. This can be an issue for humid indoor air when there are cold surfaces, for example, windows. A cold window surface can cool the indoor air with which it is in contact and lead to condensation if the air is cooled below its dew point temperature.

- The wet-bulb temperature is the temperature that a quantity of air can be cooled to through addition of moisture to $100 \%$ relative humidity. To expand on the example of the $72^{\circ} \mathrm{F}$ indoor air at $25 \%$ relative humidity, if moisture were added to this air without any additional source of heating until the relative humidity rose to $100 \%$, the air would cool to about $50^{\circ} \mathrm{F}$. So, in other words, the wet-bulb temperature of air at $72^{\circ} \mathrm{F}$ and $25 \%$ relative humidity is $50^{\circ} \mathrm{F}$.

Air-conditioner coefficient of performance (or COP effect):

- The COP is the instantaneous ratio of beneficial cooling energy delivered to the air stream from the evaporator divided by the electric energy input to the compressor. COPs for airconditioning systems can range from 2 to 6 (or higher) depending on the quality of the refrigeration cycle's components and the indoor and outdoor temperatures. A COP of 4 is the same as saying that the system is $400 \%$ efficient at converting electricity to cooling energy at current conditions. Some confusion arises because, at first glance, this appears to violate thermodynamic principles (the first law of thermodynamics). However, the proper thermodynamic analysis includes the entire system, such that the energy added to the refrigerant must equal the energy that leaves the refrigerant. This means:

\section{[Evaporator heat gain + Compressor electric input] = Condenser heat rejection}

- There are practical limits to the COP, but in general, it is important to understand that the refrigeration cycle provides much more useful energy in heating/cooling than it takes to run the refrigeration cycle's "pump" (the compressor). The energy efficiency ratio (EER) is another measure of instantaneous energy efficiency, measured as the steady-state rate of heat energy removal (or cooling capacity, in British thermal units per hour [Btu/hr]) divided by the steady-state energy input to the equipment (in watts). 
COP and EER are measures of instantaneous efficiency. Other measures include the following:

- The SEER (seasonal energy efficiency ratio) is a measure of the efficiency of the entire system over the course of an entire cooling season in a typical temperate climate. The SEER is defined as the Btus of cooling energy per watt-hour of electricity consumption (including both the compressor and supply fan). While most residential-sized equipment is rated using the SEER, larger units are most likely rated using the EER or IEER (integrated energy efficiency ratio).

- The IEER (integrated energy efficiency ratio) is a part-load cooling efficiency metric that replaced the integrated partial load value in 2010 and applies to unitary equipment rated at greater than or equal to $65,000 \mathrm{Btu} / \mathrm{hr}$ (or 5 tons). It is calculated by operating the system at four different capacities and temperature conditions and applying a formula to determine the overall ratio. ${ }^{13}$

- A SEER of 14.5 (equivalent to an average seasonal COP of 4.25) for split systems or an IEER of 14 (average seasonal COP of 4.10) for packaged systems is considered energy efficient, and equipment with this rating or higher qualifies to carry the Energy Star label $\left.\right|^{14}$ To convert from EER (SEER or IEER) to the dimensionless efficiency metric, COP, multiply the EER by 0.293.

Heat pump heating efficiency:

- Heating efficiency from a heat pump is defined by its heating season performance factor (HSPF). This factor is based on a standard test procedure to identify how much heating energy is delivered on average over the heating season (output) per unit of electric energy input to the compressor in a typical seasonal climate.

- Like the IEER, this measure is defined as the Btu of heat energy per watt-hour of electricity consumption. HSPFs over 8 for packaged equipment and over 8.2 for split systems are considered energy efficient and qualify to carry the Energy Star label. To convert from HSPF (or IEER) to the dimensionless efficiency metric, COP, multiply the HSPF by 0.293. Thus, a heat pump with an HSPF of 8 has an average COP over the heating season in most climates of 2.34, or is about 2.34 times more efficient at heating than an electric resistance heating coil. For units greater than 5 tons in capacity $(65,000 \mathrm{r} / \mathrm{h})$, there may only be the COP rating and not the HSPF rating.

\footnotetext{
${ }^{13}$ EEER $=(0.02 * \mathrm{~A})+(0.617 * \mathrm{~B})+(0.238 * \mathrm{C})+(0.125 * \mathrm{D})$ where $\mathrm{A}=\mathrm{EER}$ at $100 \%$ net capacity at Air-Conditioning, Heating, \& Refrigeration Institute (AHRI) standard condition (95 degrees F), B = EER at 75\% net capacity and reduced ambient $\left(81.5^{\circ} \mathrm{F}\right), \mathrm{C}=\mathrm{EER}$ at $50 \%$ net capacity and reduced ambient $\left(68^{\circ} \mathrm{F}\right)$ and $\mathrm{D}=\mathrm{EER}$ at $25 \%$ net capacity and reduced ambient $\left(65^{\circ} \mathrm{F}\right)$

${ }^{14}$ See Energy Star website for more information on Energy Star air-source heat pump product criteria:

http://www.energystar.gov/index.cfm?c=airsrc_heat.pr_crit_as_heat_pumps.
} 

Prepared by Pacific Northwest National Laboratory for the U.S. Department of Energy

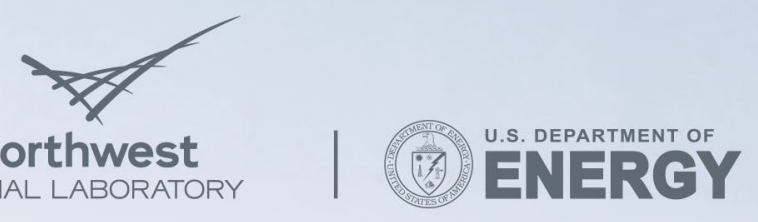

roudly Operated by Battelle Since 1965 